Illinois State University

ISU ReD: Research and eData

Theses and Dissertations

$2-1-2016$

\title{
Using A Visitor Based Framework To Observe Engagement In A Children's Museum Makerspace
}

Sara McCubbins

Illinois State University, samccub@ilstu.edu

Follow this and additional works at: https://ir.library.illinoisstate.edu/etd

Part of the Education Commons, and the Other History of Art, Architecture, and Archaeology Commons

\section{Recommended Citation}

McCubbins, Sara, "Using A Visitor Based Framework To Observe Engagement In A Children's Museum Makerspace" (2016). Theses and Dissertations. 526.

https://ir.library.illinoisstate.edu/etd/526

This Dissertation is brought to you for free and open access by ISU ReD: Research and eData. It has been accepted for inclusion in Theses and Dissertations by an authorized administrator of ISU ReD: Research and eData. For more information, please contact ISUReD@ilstu.edu. 


\title{
USING A VISITOR BASED FRAMEWORK TO OBSERVE ENGAGEMENT IN A CHILDREN'S MUSEUM MAKERSPACE
}

\author{
Sara McCubbins
}

\section{Pages}

Many challenges still exist in finding ways to measure the impact of informal learning environments. Much of the research that does exist is anecdotal in nature and examines engagement by intuition or informal feedback. The purpose of this concurrent mixed methods study was to better understand engagement and learning by converging both quantitative and qualitative data. In the study, an observation protocol was used to measure the engagement levels of children in a museum makerspace, and field notes were collected to explore the context in which this engagement takes place. The observation protocol used in this dissertation was the Visitor Based Learning Framework (VBLF) developed by Barriault and Pearson (2010) to generate insights into the impact of science center exhibits on visitor learning experiences. Participants of this study include childparent/guardian pairs who visited the chosen museum setting during its makerspace hours. The study asks (1) at what levels, based on the VBLF, do children engage with the makerspace exhibit in the museum; (2) what relationships exist between engagement levels and age and gender; and (3) how do makerspace engagement levels compare to average engagement levels of traditional museum exhibits. In addition to the quantitative 
data collected from the observation protocol instrument, qualitative field notes helped to contextualize the quantitative data. The study showed that breakthrough engagement was where it was expected to be based on previous research, at 51\%, though this was not statistically significant. However, qualitative data helped provide insight into factors that influenced breakthrough levels, such as parent dialogue, participant interest in the activity, and facilitator knowledge. This demonstrates a similarity to traditional museum exhibits in that some makerspace activities will bring a majority of participants to the breakthrough level while others will not. The findings also show that while this instrument can determine the effectiveness of individual makerspace activities in allowing participants to reach breakthrough engagement levels, it cannot determine the effectiveness of the makerspace overall without consideration for the individual activities taking place and other critical factors that are not assessed by the instrument.

KEYWORDS: Education, Museum Studies, Makerspaces, Informal Learning, Nonformal Learning, Visitor Based Learning Framework 
USING A VISITOR BASED FRAMEWORK TO OBSERVE ENGAGEMENT

IN A CHILDREN'S MUSEUM MAKERSPACE

SARA MCCUBBINS

A Dissertation Submitted in Partial

Fulfillment of the Requirements

for the Degree of

DOCTOR OF EDUCATION

School of Teaching and Learning

ILLINOIS STATE UNIVERSITY

2016 
(C) 2016 Sara McCubbins 
USING A VISITOR BASED FRAMEWORK TO OBSERVE ENGAGEMENT

IN A CHILDREN'S MUSEUM MAKERSPACE

SARA MCCUBBINS

COMMITTEE MEMBERS:

Ryan Brown, Chair

Allison Antink Meyer

Sandra Osorio 


\section{CONTENTS}

Page

CONTENTS

TABLES $\quad$ iv

FIGURES $\quad$ v

CHAPTER

I. INTRODUCTION OF THE STUDY 1

Statement of the Problem 1

Purpose of the Study $\quad 3$

Methodology 4

Definition of Terms 6

Informal Learning Environment 6

Makerspaces and the Maker Movement $\quad 12$

Visitor Based Learning Framework 13

Description of the Study 15

Limitations of the Study 16

$\begin{array}{ll}\text { Chapter Overview } & 17\end{array}$

II. REVIEW OF RELATED LITERATURE 19

Theoretical Framework $\quad 20$

$\begin{array}{ll}\text { Constructivism } & 21\end{array}$

Understanding the Learner 25

Learner Agenda and Motivation $\quad 25$

Sense-Making and Dialogue $\quad 27$

Positioning the Learner and Developing Identity 28

Understanding the Museum Setting 30

Exhibit Design: Labeling, Aesthetics, and Interaction 30 
Summary

Statement of the Problem 44

Research Design $\quad 45$

Site Selection $\quad 49$

Participant Selection $\quad 51$

Observations $\quad 52$

$\begin{array}{lr}\text { Instrument } & 54\end{array}$

Development $\quad 54$

Observational Behaviors $\quad 55$

Validity and Reliability $\quad 58$

$\begin{array}{ll}\text { Data Collection } & 60\end{array}$

Data Analysis $\quad 60$

Ethical Considerations $\quad 62$

IV. RESULTS, ANALYSIS, AND DISCUSSION 64

Results and Discussion $\quad 64$

Research Question One $\quad 64$

Overall Engagement Level 64

Individual Engagement Levels 66

Initiation $\quad 66$

Transition $\quad 67$

Breakthrough 68

$\begin{array}{ll}\text { Engagement Level by Activity } & 70\end{array}$

Hydraulics $\quad 70$

Squishy circuit $\quad 72$

Stop motion animation $\quad 75$

$\begin{array}{ll}\text { Influences on Engagement } & 77\end{array}$

Influence of parental dialogue $\quad 77$ 
Influence of pedagogical approach $\quad 82$

Influence of exhibit layout $\quad 84$

Research Question Two $\quad 85$

Engagement Level by Gender $\quad 86$

Engagement Level by Age Group $\quad 91$

Research Question Three 95

Summary 98

V. SUMMARY, CONCLUSIONS, AND

RECOMMENDATIONS 102

Overview of the Study 102

Summary of Findings 104

$\begin{array}{ll}\text { Conclusions } & 108\end{array}$

Implications and Strategies 109

Implications for Museum Educators $\quad 110$

Implications for Practice 111

Recommendations for Future Research 112

$\begin{array}{ll}\text { REFERENCES } & 116\end{array}$

APPENDIX A: Visitor Based Learning Framework Observation Protocol 127

APPENDIX B: Makerspace Coded Field Notes for Visitor 13

APPENDIX C: List of Emergent Codes with Comments 132

APPENDIX D: Description of Makerspace Activities by Theme 136 


\section{TABLES}

Table

1. A Summary of the Research Study Design

2. The Visitor Based Learning Framework from Barriault and Pearson (2010)

3. Frequency of Overall Engagement Behavior Levels for All Participants

4. Frequency of Individual Engagement Behavior Levels for All Participants

5. Frequency of Individual Engagement Behavior Levels for Hydraulics Activity

6. Frequency of Individual Engagement Behavior Levels for Squishy Circuit Activity

7. Frequency of Individual Engagement Behavior Levels for Stop-Motion Animation

8. Chi-Squared Test Statistics and Significance for Learning Behaviors by Gender

9. Chi-Squared Test Statistics and Significance for Learning Behaviors by Age Group

10. Correlations Among Age, Gender, and Learning Behaviors

11. Chi-Squared Test Statistics and Significance for Learning Behaviors 


\section{FIGURES}

$\begin{array}{lll}\text { Figure } & \text { Page }\end{array}$

1. Makerspace Workbench at a Children's Museum 50

2. Overall VEP for Hydraulics Makerspace Activity 71

3. Overall VEP for Squishy Circuit Makerspace Activity 73

4. Overall VEP for Stop-Motion Animation Makerspace Activity 75

5. Percentage of Participants within Each Age Group 86

6. Overall VEP for Learning Behaviors Based on Gender 87

7. A Comparison of Gender and Referring to Past Experiences Breakthrough Behavior $\quad 88$

8. Overall VEP for Learning Behaviors Based on Age 92

9. Overall VEP for the Makerspace Exhibit 96 


\section{CHAPTER I \\ INTRODUCTION OF THE STUDY}

\section{Statement of the Problem}

The Partnership for $21^{\text {st }}$ Century Skills (2011) explains in their Framework for $21^{\text {st }}$ Century Learning that in order for students to succeed in the new global economy, they must learn essential skills such as creativity and innovation, critical thinking and problem solving, and communication and collaboration within the context of their core knowledge. Yet, Pajak, Stotko, and Masci (2007) argue that when it comes to teaching, "the innovation, creativity, and democratic goals of caring, inventing, and inspiring teachers are usually de-emphasized or even implicitly discouraged" (p. 113). While the authors strive to make an argument for the ways in which we might be able to make some progress in the formal education setting by placing more value on those diverse teaching styles, they fail to examine the important role informal learning environments such as museums, zoos, science centers, and aquaria play in the development of these skills. Qualities such as innovation, creativity, and collaboration are staples of informal learning environments (Bailey et al., 2016). In relation to science, these qualities manifest themselves in activities that aim to integrate disciplines, such as Science, Technology, Engineering, and Mathematics (STEM) in education. In these informal spaces, resources for learning are incredibly diverse, allowing the opportunity to capture the interest of every learner (Harlow, 2012). For example, Falk and Adelman (2003) conducted a study 
of aquarium visitors. They found that people who went into the aquarium with little or no interest in conservation left with higher interest after visiting a conservation exhibit within the aquarium. Enjoyment and interest in science are often at the center of informal science design and have been found to influence attention, goals, and conceptual knowledge (Harlow, 2012; Hidi \& Renninger, 2006). Furthermore, informal learning environments can also have a positive influence on interest and career choice, even after a single informal learning experience, by increasing student attitude toward science and making children more interested in potential STEM careers (Noel-Storr, Mitchell, \& Drobnes, 2010; Bailey et al., 2016).

Most of the research surrounding informal science supports the idea that the informal learning environment is a rich source for nurturing curiosity, motivation, and attitudes toward science, and that it generates excitement and enthusiasm among its visitors (Bell et al., 2009; Braund \& Reiss, 2004; Griffin, 2004; Stocklmayer \& Gilbert, 2002). Yet, measuring the learning that occurs and the impact of informal environments is incredibly challenging. Barriault and Pearson (2010) explain that "it cannot be accomplished with the same methods that are used in formal instructional settings where participation is mandatory and the learning objectives are explicit and strongly focused on cognitive gains" (p. 91). As such, assessments of the contributions of informal learning environments to student learning and development do not occur as often as they should. And even if they do take place, the instruments used in measuring their effect are often time-, and money-, intensive (Barriault \& Pearson, 2010, p. 91). 


\section{Purpose of the Study}

Challenges exist in finding ways to measure the impact of informal learning environments. Much of the research that does exist is anecdotal in nature and examines engagement by intuition or informal feedback (Jensen, 2014). Children's museums in particular are prone to this, adding new exhibits and programs often based simply on visitor or parental feedback, or based on trending programs in other museums (Family Learning Forum, 2013). For example, the popularity of the maker movement, a grassroots movement of innovation in which communities are coming together to freely share ideas and create new things, has led to the development of many makerspaces within Children's museums. According to Mark Hatch (2014), CEO and founder of TechShop, a makerspace is defined as "a center or workspace where like-minded people get together to make things (p. 13). They range from highly-technical, such as 3D printing and lathe machinery, to craft-oriented such as sewing, to mechanical skill-focused, such as building with wood and nails. In many of the Children's Museums that I have visited, the makerspace typically consists of work benches with tools. Visitors can use any materials they can find in the space to create whatever they want. Sometimes programming accompanies the makerspaces, but often visitors are provided open access. Prompts and examples are often provided for those who need some help getting started with their creations. In addition, some makerspaces have visitors building their own individual projects while others have collaborative projects that visitors can add onto within the space.

Understanding how students are becoming engaged outside of the formal education environment is even more important given that high-stakes testing has led to a 
narrowing of curricular content, fragmentation of subject-area knowledge, and increased reliance on teacher-centered pedagogies (Au, 2007). Furthermore, as the makerspace movement continues to grow, it is important to identify whether there are useful benefits for including makerspaces, which tend to be a resource- and time-intensive exhibit in Children's Museums (Halverson \& Sheridan, 2014). Makerspaces in museums such as the Children's Museum of Pittsburgh and the New York Hall of Science have shown how the maker movement can be accessible to young people and families, and their "focus on maker culture is a natural extension of a long-standing emphasis on learning-by-doing in art and science" (Halverson \& Sheridan, 2014, p. 499). However, few of these spaces are being studied for their impact.

Given the relatively new inclusion of makerspaces in Children's Museums and the lack of research that currently exists, a number of questions arise. Understanding the level of engagement with the makerspace will help us to better understand how learning might be occurring in museum makerspaces and provide guidance for the effectiveness of future exhibits without the need for programmatic and staff-intensive scaffolding. Additionally, understanding how demographic characteristics influence engagement outcomes will provide insight into the need for potential interventions or models for success. Therefore, the purpose of this concurrent mixed methods study was to better understand engagement and learning by converging both quantitative and qualitative data.

\section{Methodology}

In the study, an observation protocol developed by Barriault and Pearson (2010) known as the Visitor Based Learning Framework (VBLF) was used to measure the 
engagement levels of children in a museum makerspace. At the same time, field notes were collected to explore the context in which this engagement takes place. The following research questions were used to guide this study:

1. At what levels, based on the VBLF, do children engage with the makerspace exhibit in the museum?

2. What relationships exist between engagement levels and age and gender?

3. How do makerspace engagement levels compare to average engagement levels of traditional museum exhibits?

Because of its ability to allow for an expansion of understanding from one method to another, and because they present the ability to converge or confirm findings from different data sources (Creswell, 2003), a mixed-method approach is the best method for answering these research questions and was chosen for this study.

In addition to the observational data collected for the visitor based learning framework, detailed field notes were recorded for the visitors for the duration of their time in the makerspace exhibit. In these field notes, observations on interaction and engagement were recorded, along with dialogue and activity. Analysis focused on repeating themes that emerged over the course of the observations. According to Mack, et. al. (2005), field notes should be a record of what you experience and learned from your observation of others, and they should include behaviors, reactions, conversations, physical gestures, and other detailed observations of your participants in order to complete a full picture of the experience (p. 21). In this study, the makerspace themes changed every week or two. In total, five different themes were observed: pollinators, hydraulics, open lab, squishy circuit, and stop-motion animation. As a result, the field 
notes on the first day of a new theme were more detailed in order to describe the new setting and focus of activities. After the initial description, field notes were focused less on the specific activity taking place and more on the visitor's encounter with that activity.

\section{Definition of Terms}

To address these research questions, it is important to understand some key terminology for this study. The paragraphs that follow provide definitions and background information for the informal learning environment, makerspaces and the maker movement, and the visitor based learning framework, within the context of this study.

\section{Informal Learning Environment}

The literature presents a variety of ways to define the informal learning environment, and often researchers take slightly different approaches that will suit the needs of their study. Informal learning is almost always defined in opposition to the formal learning environment, representing any kind of learning that is taking place outside of a formal classroom. Sometimes the environment is defined based on the kinds of learning and activities taking place. Research has been conducted on informal learning environments such as field trips (Griffin \& Symington, 1997; Rix \& McSorley, 1999), scouting activities (Jarman, 2005), summer enrichment programs (Jones, 1997), science practices at home (Hall \& Schaverien, 2001), and family visits to museums (Ash, 2004), to name a few.

Even the terminology used has its own variability. For instance, research shows only slight differences, and often interchangeability, between informal, non-formal, and free-choice learning. The majority of the literature seems to embrace the definition of 
informal learning provided by Falk and Dierking (2000), two prominent researchers in the field, who describe it as one in which "learning tends to be non-linear, is personally motivated, and involves considerable choice on the part of the learner as to what to learn, as well as where and when to participate in learning" (as cited in Barriault \& Pearson, 2010, p. 13). According to Barriault and Pearson (2010), these environments, which include spaces such as museums, science centers, zoos, and aquaria, often offer realworld examples of science and explorations of phenomena, where "people can pursue and develop science interests, engage in science inquiry, and reflect on their experiences through sense-making conversations" (p. 91). The definition of the informal learning environment is further expanded by researchers Storksdieck, Jones, Falk, and Alpert (2002), who demonstrate that "the free-choice learning sector affords people the opportunity for lifelong, voluntary, intrinsically motivated and largely self-chosen and self-controlled learning" (p. 5). Ultimately, despite the nuanced range of venues, learners, objectives, and even definitions, all informal learning research is united by the idea that such learning occurs as opportunities beyond the formal education environment (Phipps, 2010).

Given that informal learning is often positioned as "not" formal learning, it is important to recognize how the informal environment is different from the formal environment. As Castle (2006) explains, "While not a school, a museum does encompass deliberately educational activities. A museum can be said to have a curriculum in the broadest sense of the word - curriculum as a medium by which educators attempt to represent what happens or what ought to happen in educational practice and how it is to be achieved" (p. 123). That being said, the formal classroom does not always offer the 
flexibility necessary to promote educational goals in the way that an informal environment can. Paris (1997) explains, "it is ironic that one of the goals of education is to foster independent learning, yet students are often given few opportunities to select and control their own learning environments" (p. 24).

Control of the learning environment is important not only for interest and attitude toward science but also for content knowledge acquisition. When a visitor is interested in a topic, he or she is more likely to interact longer and take away more information than when that visitor is not interested in that topic or exhibit (Paris, 1997). Most people learn and understand science not from the formal environment but rather from outside of school (Storksdieck, Jones, Falk, \& Alpert, 2002; National Science Board, 1998). As a result, "informal science education centers such as science museums have taken an increasingly important leadership role in complementing the role of school curricula and the popular media in informing K-12 students and the public on areas of scientific knowledge and research" (Storksdieck, Jones, Falk, \& Alpert, 2002, p. 3).

Qualities such as innovation, creativity, and collaboration are often staples of informal learning environments. In relation to science specifically, these qualities often manifest themselves in activities that aim to integrate disciplines, such as STEM. Resources for learning are incredibly diverse, allowing the opportunity to capture the interest of every learner (Harlow, 2012). For example, a study by Zoldosova and Prokop (2006) looked at a five-day field trip program at the Science Field Center, a field laboratory for biological and chemical experiments in the Slovak mountains. An experimental group of students $(N=153)$ participated in biology and chemistry experiments during that time, while the control group $(N=365)$ did not experience the 
field studies. They found that the students in the experimental group, when given a list of reading materials, chose relatively more science-oriented books than their peers who did not participate in the field trips. Falk and Adelman (2003) also conducted a study of 392 aquarium visitors. After analyzing the pre- and post- interviews of 100 individuals, the results showed that people who went into the aquarium with little or no interest in conservation left with higher interest after visiting a conservation exhibit within the aquarium. As seen from these studies, the free choice learning environment greatly impacts the ability to capture the unique interests of each learner.

Enjoyment and interest in science are often at the center of informal science design and have been found to influence attention, goals, and conceptual knowledge (Harlow, 2012; Hidi \& Renninger, 2006). In a study of pre-service teachers, Harlow (2012) examined reflective postings, class discussions, observations, artifacts, and photographs and found that these teachers were able to successfully teach science and develop children's ideas about science through facilitated science stations as a family science night. Furthermore, informal learning environments can also have a positive influence on interest and career choice, even after a single informal learning experience (Noel-Storr et al., 2010). A longitudinal study by Fadigan and Hammrich (2004) examined the educational trajectories of 152 female participants from urban, low-income, single-parent families who participated in a high school informal science program called Women in Natural Sciences (WINS). The study found that of the 109 participants (93.16\%) who enrolled in a college program, 45 of them $(44.55 \%)$ designated a career path within medical/health or STEM fields. 
Informal science and STEM outreach programs also provide an opportunity to learn about science in real-world settings, something that is also known as citizen science. Silvertown (2009) explains that "most citizen scientists work with professional counterparts on projects that have been specifically designed or adapted to give amateurs a role, either for the educational benefit of the volunteers themselves or for the benefit of the project" (p. 467). This is not true, however, in the formal science setting, where the dominant scientific elite have chosen curricula which reflect the scientific knowledge needed to educate the future scientist rather than the science needed for the future citizen (Claussen \& Osborne, 2012, p. 60). For example, a formal science curriculum often excludes any history of science, applications and implications of science, and discussion of how science works.

While new standards are currently being adopted nationwide that reflect a shift toward scientific practices for the everyday citizen, many students are still educated daily on science content that does not pertain to their lives and would only be useful were they to pursue a career as a scientist. This divide presents an opportunity for informal learning environments to not just try and replicate their formal counterparts by focusing so heavily on content, but to embrace other important skills as well. Storksdieck, Jones, Falk, and Alpert (2002) explain that "a large percentage of the specific knowledge individuals acquire during formal schooling wanes over time unless it is periodically practiced or renewed, although some of the more general knowledge may be retained even over a long period of time" (p. 4). Research has shown that informal learning environments are uniquely positioned to provide that periodic practice of knowledge in ways that are meaningful to the individual, while also embracing a rich learning setting that embraces 
curiosity, encourages participation, generates excitement, and improves motivation and attitude toward science (Barriault \& Pearson, 2010; Bell, Lewenstein, Shouse, \& Feder, 2009; Griffin, 2004; Ramey-Gassert, Walberg, \& Walberg, 1994; Rennie \& McClafferty, 1995; Stocklmayer \& Gilbert, 2002).

Finally, the literature highlights the importance of lifelong learning and the variability of learning for informal environments. Lifelong learning is something that was embraced heavily by educational philosopher John Dewey (1938) and was of critical importance to Mary Parker Follett, a management pioneer and theorist who "believed informal learning was nurtured at birth and spanned one's entire life, acquired by the modes of living and acting that teach an individual how to grow a social consciousness" (Conlon, 2004, p. 286). This view is consistent with the constructivist theoretical framework upon which many informal science studies are built. While not exclusive to the informal environment, the literature stressed the importance of using museums and other informal settings to stimulate and encourage lifelong learning, given their predisposition toward viewing learning with a wide lens rather than focusing solely on content (Hofstein \& Rosenfeld, 1996; Felton \& Kuhn, 2007).

As Ritchhart (2007) explains in his research on cultural forces that shape group learning, "models of thinking and learning are important for students to see as they strive to take on new ways of thinking and being in the world. When learning is focused solely on facts, skills, and knowledge, students are given an impoverished model of what it means to learn" (p. 144). Furthermore, the variability of learning, including ways of thinking as well as when learning is occurring in one's life, was a thread throughout the literature (Storsksdieck, Jones, Falk, \& Alpert, 2002; Barriault \& Pearson, 2010). This 
variability places the act of learning on the learner, something that is critical to the makerspace environment.

\section{Makerspaces and the Maker Movement}

According to Mark Hatch (2014), CEO and founder of TechShop, a makerspace is defined as "a center or workspace where like-minded people get together to make things (p. 13). They range from highly-technical, such as 3D printing and lathe machinery, to craft-oriented such as sewing, to mechanical skill-focused, such as building with wood and nails. These spaces have developed out of a maker culture, which according to Halverson \& Sheridan (2014), has "become a way to express creative and communal drive, and this excitement has led to an explosion of makerspaces around the United States (and the world) across a range of instructional environments, including libraries, museums, independent nonprofit and for-profit organizations, K-12 schools, and institutions of higher learning” (p. 495).

These spaces can take on many forms depending on their audience and location. Spaces in museums and libraries have a very different focus than spaces that are designed for commercial or private use. Often, the focus on museum and library makerspaces is on promoting creativity and the sharing of resources (Honey \& Kanter, 2013). More industrial spaces are often focused on not just practicing skills but also expanding them and developing expertise that can benefit the entire maker community (Sheridan, et al., 2012). In addition to serving different audiences, these spaces also have different visions for their space. As Halverson \& Sheridan (2014) explain, "some spaces focus on the emergence of new technologies for designing, building, and manufacturing, while others value the return to face-to-face, garage-style work that the maker movement engenders" 
(p. 495). In the end, these spaces form a community of people inclined toward maker culture, which is directly connected to the maker movement, a movement that is rapidly expanding across the country.

Halverson \& Sheridan (2014) explain that the maker movement can be broadly defined as a "growing number of people who are engaged in the creative production of artifacts in their daily lives and who find physical and digital forums to share their processes and products with others" (p. 496). Many makerspaces follow the guidelines laid out by Mark Hatch, who is the CEO and founder of TechShop, one of the first makerspaces, and who wrote a "manifesto" that organizes maker mindsets around nine ideas: make, share, give, learn, secure tools, play, participate, support, and change (Hatch, 2014). In higher education, the maker movement originated with the creation of fabrication labs, or FabLabs, started first by a professor at the Massachusetts Institute of Technology. Gershenfeld (2005) wanted to create a pedagogical environment that would support the fabrication of tools needed by people to solve their own problems, rather than purchasing or outsourcing those tools. As the maker movement transitioned into informal learning environments such as museums, makerspaces became more flexible and able to reach a broader audience because the focus shifted towards tools and processes that were more readily available to those who might not normally self-identify as a maker (Kafai, Fields, \& Searle, 2014). This broadness and flexibility of the maker movement is what allows it to stretch across the divide between formal and informal learning environments.

\section{Visitor Based Learning Framework}

For the purpose of this study, a visitor based learning framework was used for data collection. The observation protocol, originally developed by Barriault (1999) and 
then refined by Barriault and Pearson (2010), can be found in Appendix A and was used to document students' stages of learning (whether they are at the initiation, transformation, or breakthrough stages of learning) as they engage with a new “makerspace" exhibit at a local children's museum. The instrument has been used for more than 10 years and is seen as "a practical tool for science center staff to easily understand the impact the exhibits have on visitors' learning behaviors" (Barriault \& Pearson, 2010, p. 98). In describing the instrument's validity and reliability, Barriault explained:

It is important to note that the learning behaviors and engagement levels in the instrument are a proxy for learning and an indication of engagement. Together, they serve as an assessment tool that measures the potential learning impact of the exhibits being observed. In other words, the occurrence of learning behaviors indicate that the conditions and opportunities presented by the exhibit are conducive to engaging visitors in a learning experience (C. Barriault, personal communication, April 28, 2015).

The observation protocol includes seven learning behaviors which are grouped into three categories — initiation, transition, and breakthrough — each reflecting increasing engagement. Initiation behaviors are the behaviors of doing or of watching others engaging. These behaviors indicate that the visitor is taking preliminary steps toward meaningful engagement. As Barriault (2008) explains, these behaviors "enable visitors to 'test the waters' with minimal personal risk and provide an entry point into further learning opportunities offered by the exhibit" (p. 17). At the transition level, the behaviors of repeating the activity and of demonstrating a positive emotional response are observed. These behaviors demonstrate increased engagement and show that the visitor has an increased level of comfort with the exhibit, which leads to more commitment and motivation toward learning (Barriault, 2008). Finally, the researcher is looking for 
behaviors of referring to past experience, seeking and sharing information, or being engaged and involved (i.e. testing variables, making comparisons, using information gained from the activity), all of which exhibit breakthrough behaviors. At this level, real learning can occur because the visitor has established a personal level of comfort allowing a free exchange of ideas and is demonstrating an acknowledgement of "the relevance of the activity, and the learning gained form the activity, to the individual's everyday life" (Barriault, 2008, p. 18).

These observations are then quantified into what is described by Barriault (1999) as a Visitor Engagement Profile (VEP), in which the y-axis represents the percentage of visitors who engage in the learning behaviors on the $\mathrm{x}$-axis. It is important to note that in all of these graphs, the initiation behavior is at $100 \%$ because of the fact that only those who approach the exhibit are counted or observed. As Barriault (2008) explains, "The VEP is not intended to be a measure of the attracting power because of the challenge of assessing the reasons why particular visitors did not approach the exhibit. Instead, the VEP focuses our attention on the learning behaviors demonstrated by visitors once they have made the commitment to engage with the exhibit" (p. 20). While an ideal curve depends on the type of exhibit being assessed, Barriault (2008) explains that exhibits which are designed to involve visitors in hypothesis testing and meaning making such as the makerspace should produce a VEP with at least $50 \%$ of visitors demonstrating breakthrough behaviors.

\section{Description of the Study}

This study uses a mixed methods design to examine children's engagement levels and science learning when they participated in a makerspace exhibit. Mixed-methods 
techniques incorporate components of both qualitative and quantitative data to offer a variety of perspectives on a research study and are becoming more prevalent. As Creswell (2003) explains, "with the development and perceived legitimacy of both qualitative and quantitative research in the social and human sciences, mixed methods research, employing the data collection associated with both forms of data, is expanding" (p. 208). In this case, an observation protocol was used, leading to an overt naturalistic observational design in which the researcher role was explicitly stated but the researcher remained in the background during observations (Vogt, Gardner, \& Haeffele, 2012). Quantitative data was generated from the observation protocol components and was used to answer research questions one, two, and three. Qualitative data was generated from extensive field notes, which were recorded for each participant. The focus of these field notes was to observe the engagement of the visitors and their interactions within the makerspace in order to support the quantitative data for each research question.

\section{Limitations of the Study}

One of the major limitations to this study is the inability for a single researcher to collect all the possible data given the intensive nature of the observation protocol and the fact that no audio/visual recordings were used. To help address this limitation, only two visitors were observed at any given time. While not feasible for this study, these limitations could be further addressed in future studies by including audio/visual recording to collect data as well as increasing the number of researchers collecting observational data in order to develop reliability between the researchers and the data collected. Another limitation is that the Visitor Based Learning Framework was developed in and for science centers as an assessment tool for science-focused exhibits. 
Some exhibits are static, which presents different opportunities for engagement than an active "exhibit" such as a makerspace.

Although additional studies have been done on the framework to strengthen confidence in its generalizability and validity (Barriault, 2014), this is the first time the framework has been used in both a Children's museum setting and a makerspace setting. In both of these cases, there is the potential for visitors to experience longer engagement cycles which could factor into the number of visitors reaching the breakthrough level of engagement. More studies are needed to determine if this framework can truly represent engagement levels in these settings in the same manner that it does in science centers and zoos/aquaria. Finally, another limitation to this study is consistency in coding for the experiences to determine engagement levels. With only one observer, there was no interrater reliability. However, efforts were made to refine observations, remove ambiguities, and consistently code the behaviors observed.

\section{Chapter Overview}

In this chapter, a brief review of relevant literature provided the foundation for this study and contextualized its significance. An overview of the methods and research design was also provided and limitations were presented. In chapter two, the theoretical framework of the study will be outlined. The chapter will also review important research and literature that situates the study within the context of understanding the learner and understanding the museum setting. An in-depth review of the Visitor Based Learning Framework will also be provided. Chapter three will re-examine the problem and describe the research design. Instrumentation, quantitative and qualitative techniques, data analysis, and ethical considerations will all be addressed in this chapter. Results, 
analysis, and discussion will comprise chapter four. Qualitative analysis will focus on the breakdown of each behavior level while quantitative analysis will focus on descriptive analytics, correlations, and chi-square analysis. Finally, chapter five will provide a summary of the findings as well as implications and recommendations for future research. 


\section{CHAPTER II}

\section{REVIEW OF RELATED LITERATURE}

The United States has long sought after the goals of increasing science awareness among its citizens, promoting developments in scientific research and education, and encouraging participation in STEM (science, technology, engineering, and mathematics) careers (DeBoer, 2000). Fueled by the launch of Sputnik in the late 1950's, this nation has aggressively worked to establish itself as a world leader in research and education within the STEM fields. Such national reform revealed itself through rigorous development of programs such as the National Science Foundation (NSF) and the National Aeronautics and Space Administration (NASA) to help promote scientific research. While part of the school curriculum since the $19^{\text {th }}$ century (DeBoer, 2000), standards for the STEM disciplines, and particularly science, did not make their way into science education reform until the development of the National Science Education Standards by the National Research Council in the early 1990s. These standards helped to outline important benchmarks in order to produce a scientifically literate society, and since then, researchers have been increasingly interested in examining how the public understands and appreciates science (National Science Board, 1998; 2000; 2002).

Much of the focus on public understanding of science has been directed toward the formal education sector, but Hofstein and Rosenfeld (1996) explain that "human beings learn science from a variety of sources, in a variety of settings, and for a variety of 
reasons" (p. 88). Informal settings such as museums and science centers often offer opportunities for visitors to experience science outside of the classroom setting, focusing on developing skills rather than specifically targeting content knowledge. While content knowledge might be the focus of many standardized high-stakes tests, it is not the only knowledge that is believed to be important for the future. The Partnership for $21^{\text {st }}$ Century Skills (2011) explains in their Framework for $21^{s t}$ Century Learning that in order for students to succeed in the new global economy, they must learn essential skills such as creativity and innovation, critical thinking and problem solving, and communication and collaboration within the context of their core knowledge (p. 1). Museums and science centers seem to be uniquely positioned to provide visitors with opportunities to develop these essential skills because of their free choice learning environment which removes many time and content constraints typically felt by the traditional classroom. However, research in informal learning tends to be scattered across a wide variety of disciplines and interests, from education to sociology to cognitive science to museum studies, and a clear research agenda for the field is not immediately obvious. In the following chapter, a theoretical framework for this study will be provided, and related literature will be reviewed, specifically focusing on our understanding of the learner and of the museum setting.

\section{Theoretical Framework}

Early studies in informal learning often lacked a conceptual framework in which to guide the study (Dierking \& Martin, 1997), and as a result, informal learning was often viewed as a sub-par research area to learning in a formal context. However, over the last decade and a half, the literature placed a greater focus on theoretical underpinnings, and 
the majority of studies published in informal learning over the last decade have focused on two theoretical frameworks: constructivism and sociocultural theory (Phipps, 2010). Sociocultural theory is a theory that "emphasizes that meaning emerges in the interplay between individuals acting in social contexts and the mediators-including tools, talk, activity structures, signs, and symbolic systems - that are employed in those contexts" (Schauble, Leinhardt, \& Martin, 1997, p. 4). It is strongly tied to understanding the visitor's motivations and interests as well as their sense of identity. Previous studies have explored sociocultural theory within an informal learning context by examining how learners use cognitive tools to mediate their thinking (Hohenstein \& Tran, 2007) as well as how group activity has played a role in learning (Ash, 2003). But, as museums shifted toward a focus on hands-on learning, researchers framed their studies in constructivism, specifically examining how learners changed their conceptual models (Anderson, Lucas, \& Ginns, 2003) and the learner's prior knowledge (Lucas, 2000).

\section{Constructivism}

Because of the natural way in which makerspaces frame learning as experimentation and authentic inquiry (Halverson \& Sheridan, 2014), this study was developed under the theoretical framework of Constructivism. According to Bodner (1986), constructivist theories of knowledge are based on the fundamental believe that knowledge is constructed in the mind of the learner. Constructivist theories of knowledge also require a subtle shift for the individual at the front of the class as someone who teaches to someone who facilitates learning (Bodner, 2001). This theory of constructivism arose in part from Piaget's Theory of Cognitive Development, which "claimed a significant and permanent presence in education during the last quarter of the 
$20^{\text {th }}$ century as it ushered in the field of cognitive science" (Nurrenbern, 2001, p. 1107). Constructivism itself is similar to Piagetian theory, as Bunce (2001) points out, in that it focuses on student-centered learning, assimilation of new knowledge with pre-existing knowledge and the resulting accommodation of that new knowledge within the student's understanding (1107-2). Student-centered learning in which learners are active participants is key to constructivist theory, and challenges the notion of students as empty vessels waiting to be filled with knowledge. Such notions are still widely applied in many educational situations (Nurrenbern, 2001).

Assimilation and accommodation of information are also important foundations of constructivism and Piagetian theory in that they explain how new information becomes incorporated into the mind of the learner (Piaget, 1954). New knowledge can only be retained by assimilating the knowledge—-fitting the knowledge into a previously constructed category or schema — or accommodating the knowledge—adjusting the constructed category or schema to include the new knowledge (Piaget, 1954). In order for this process to occur, this new knowledge must be of value to the learner. Nurrenbern (2001) explains, "If a child's existing, but limited, knowledge is acceptable or sufficient to him or her for understanding or explaining some phenomenon... then the child must experience some event that perturbs or disequilibrates this knowledge state before considering it worthwhile to restructure his or her knowledge schemas" (1109). This, in part, is what makes the learning meaningful for the student. According to Lowery-Bretz (2001), "assimilating new concepts not only provides meaning for this new information, but can also restructure (sometimes slightly, sometimes significantly) the meanings of the previously known concepts" (1107-6). In order for meaningful learning to take place, 
three conditions must be satisfied: a student must have some prior knowledge to which new information can be related; the new information must be meaningful in and of itself; and the students must consciously choose to incorporate this meaningful new information into his/her existing knowledge. According to Lowery-Bretz (2001), "meaningful learning requires the student to detect similarities and differences between new and existing knowledge, and in doing so, tackle the difficult challenge of...constructing learning" (1107-6). It is through this process of constructing knowledge that one can see the ways in which constructivism and informal learning go hand in hand.

Much of the research literature within informal learning speaks to the importance of prior knowledge and holds its footings in the theoretical framework of constructivism. In a museum setting, the learner is assumed to arrive with preconceived ideas and experiences rather than as empty vessels or blank slates (Barriaut \& Pearson, 2010; Felton \& Kuhn, 2007; Dudzinska-Przesmitzki \& Grenier, 2008). As Jeffery-Clay (1998) highlights, "museums may be the perfect environment in which to use constructivist theory and observe meaningful learning" (p. 5). Felton and Kuhn (2007), in their study on the importance of critical thinking, explained the importance of prior knowledge and experience, stating that both children and adults "bring with them a wealth of prior knowledge, assumptions, skills, and dispositions, all varying enormously across individuals and all likely to have a profound impact on what meaning museum visitors take out of their experience" (p. 101).

Prior knowledge and experience can greatly influence not only what exhibits the visitors are attracted to but also how meaningful their learning experience might be, which can ultimately affect their affinity toward science. Research by Storksdieck, Jones, 
Falk, and Alpert (2002) demonstrated that "people show a greater interest and feel better informed and more knowledgeable about issues they are more likely to experience," and that "many science-related issues are not very close to an average person's daily life, despite the fact that science permeates society" (p. 26). As visitors interact with various exhibits and learn new or competing information, they have to decide what they will do with that information, the process which Piaget referred to as "assimilation or accommodation." A study by Felton and Kuhn (2007) suggested that when students are presented with new information, "they may respond to what they see by dismissing it, by assimilating it into what they already know, or by adding to, elaborating, and even transforming their existing understanding. " (p. 101). The ability to construct new knowledge demonstrates the importance of prior knowledge and experience.

Constructivism plays a large role in guiding the level of interaction present in museum exhibits. While museums often contain artifacts and pieces of history to remind us of the past, more and more museums are shifting their focus to hands-on exhibits that help teach visitors about current practices as well, especially in science centers. As Crowley and Callanan (1998) explain, "hands-on exhibits provide children and parents opportunities to manipulate devices, explore possibilities, test ideas, and explain something new about the world" (p. 17). Jeffery-Clay (1998) also highlights the importance of shifting museums from object-based to experience-based visits:

The key to constructivism is not in what one does with the hands but what one does with the mind. In using constructivist theory to develop our museums, we must not take for granted the fact that we have wonderful objects that invite meaningful experiences. It is how we present those objects, how we allow visitors to interact with them, and how we help visitors relate those objects to their prior knowledge that will determine visitor learning (p. 6). 
When exhibits are not designed to be hands-on, visitors often get the wrong impression of the experience. Gutwill (2008) explains that traditional didactic exhibits "may convey the notion that scientific experimentation is nothing more than a simple sequence of "do, notice, read""' (p. 189). Furthermore, "Casting visitors in the role of mere recipients of information may leave them feeling slightly disempowered, less able to make sense of the world for themselves" (Gutwill, 2008, p. 190). Because of the active role of both the visitor and the parent in a Children's Museum makerspace, it serves as an excellent example of a purposefully designed constructivist-based exhibit and will be the focus of this study.

\section{Understanding the Learner}

Understanding the role of the learner was an important focus in the literature and can be categorized under the following topics: learner agenda and motivation, sensemaking and dialogue, and positioning the learning and developing identity. Each of these areas will be explored further in the sections that follow.

\section{Learner Agenda and Motivation}

One of the key components to the informal learning environment is the ability for the learner to set the agenda for learning. In a formal setting, the agenda is often set in advance and students have little choice in what or how they are learning (Paris, 1997). However, informal environments are different because of the free-choice nature of the experience. Research by Storksdieck, Jones, Falk, and Alpert (2002) shows that people focus on what they care about, and that "science-related information is absorbed only when it passes through a variety of filters that include personal interest, prior knowledge, a connection to their lives that renders the information meaningful, and mental models or 
schema into which information is integrated" (p. 26). Furthermore, research by Hilke (1988) has shown that "visitors are more likely to follow their own agendas, focusing on exhibits and concepts of interest to them or with which they have previous experience. Instead of making connections among various parts of an exhibit, visitors seek relationships to their own knowledge and experiences" (as cited in Jeffery-Clay, 1998, p. 6). This agenda for learning, and the control over that agenda that the informal environment provides, is largely influenced by motivation.

Motivation is a key component of the learner experience because visitors who are not motivated to learn will not be engaged in the exhibits. Many museums have engagement goals as part of their overall mission and vision. As a result, one of the primary concentrations of museum research has been on learner motivation. In a study on developing a research agenda to support a cumulative body of knowledge on museum learning, Schauble, Leinhardt, and Martin (1997) explained that "how and what people learn in museums are very much a function of their motivations (why they have come there), their interests (enduring propensities to engage with a topic), and their sense of identity (who they think they are in relation to museum offerings)" (p. 6). Research by Paris (1997) also shows a connection between motivation and interest. "Museum visitors often bring with them their own questions, issues, and interests that impel them to explore exhibits....People are motivated to learn more about topics that have personal relevance and utility" (p. 22). Inevitably, motivation and interest are closely linked and rely heavily on the prior knowledge and experiences of the learner, which will be the focus of the next section. 


\section{Sense-Making and Dialogue}

Understanding how to make sense of knowledge presented in museums is important for both the learner and the museum staff. A study by Dewitt (2008) involving 123 students from four schools showed that understanding how visitors make sense of new knowledge may also "help museum practitioners to develop experiences that capitalize on existing understandings to support further learning" (p. 165). Making meaning out of new knowledge does not always occur when museum staff wants it to, however. In a quasi-experimental study on the ways in which students make meaning and develop critical thinking from an art museum-school partnership program, Burchenal and Grohe (2007) found that "by slowing down and carefully looking at and discussing works of art, students have unique opportunities to apply previous experiences and knowledge to puzzle over meaning in the artworks--in other words, the students make meaning on their own terms" (p. 115). Ritchhart (2007) also highlighted the ways in which sensemaking resided in the hands of the learner, stating that "in museums, students are interacting with and trying to make sense of new objects and experiences and must think to do so. Specifically, they must: look closely; wonder and question; make interpretations and form hypotheses based on evidence; make connections to things they already know; consider different perspectives and viewpoints; delve below the surface to uncover complexity; and form conclusions" (p. 139).

Sense-making often relies on dialogue and conversation to interpret and critically analyze new knowledge. Research from Felton and Kuhn (2007) explain the importance of dialogue for the learner. "Visitors need to actively engage in discourse about their knowledge. Conversation is a powerful tool for museum learning, and dialogue has all 
the potential cognitive benefits" (p. 109). The literature also attends to dialogue in regards to argumentation and scientific reasoning. In a multiple case study of parent-child pairs conducted by Zimmerman, Reeve, and Bell (2008), the researchers focused on what they referred to as "epistemic moves" that were used to create meaning within a collaborative group. Such "epistemic moves" include "suggesting a topic for discussion, asking a clarifying question, making a statement of fact, re-voicing an idea, providing evidence for a claim, disputing someone else's claim, making a prediction, or sharing an observation or inference" (p. 144). All of these are important components of dialogue, but a longitudinal ethnographic study by Bricker and Bell (2014) demonstrated the importance of emotions when it comes to sense-making. The researchers found that for the parentchild pair that they studied, how the child was feeling about the learning experience was equally as important as the conversations taking place to bridge meaning making across a variety of experiences (p. 272). Whether a learner is using dialogue or feeling to help make sense of new knowledge, the experiences become enveloped in how that learner positions and identifies themselves, which is the focus of the following paragraphs.

\section{Positioning the Learner and Developing Identity}

Developing a strong self-identity as a scientist can go a long way toward building confidence and persistence within the sciences, and it is important to do so both in formal and informal settings. Bricker and Bell (2014) highlighted the importance of positioning the learner as a scientist in their longitudinal ethnographic study of Brenda and her mother, Stella. In this case, Stella is the one positioning Brenda. The researchers state:

Stella actively orchestrated most of the details related to situated events in Brenda's learning pathways. For example, by allowing and fully supporting the potion making activity when Brenda and her cousin were younger, and then by purchasing a science-related kit that was directly related to the 
potion-making interest, Stella both coordinated various details of the situated events in which Brenda participated, and positioned Brenda as a person who was competent with respect to the skills required to participate in these events (p. 275).

By allowing Brenda to see herself as a scientist, and by providing numerous opportunities in which she could be successful, Stella gave her daughter a sense of agency, confidence, and the sense of having a collaborator in whom she could trust, which research has shown to be important in developing assigned positions within social constructs (Harre, Moghaddam, Cairnie, Rothbart, \& Sabat, 2009; Holland \& Leander, 2004; Dreier, 2008). As Bricker and Bell (2014) explain, "Brenda herself identified as someone interested in aspects of the sciences, and Stella actively positioned Brenda (through her actions, talk, choice of activity, choice of settings) as someone capable of doing science-related work" (p. 267).

The importance of Bricker and Bell's research is not only that it identified the importance of positioning and identity in the informal learning environment but that it also illuminated the dichotomy that can exist between formal and informal settings. Brenda's teachers “did not recognize her, and therefore position her, as someone who was excited about science" (Bricker \& Bell, 2014, p. 280). As a result, "Brenda's rich socio-material practices related to scientific practices were largely not cued in school-except when she was given personal choice in relation to a series of science investigations" (p. 268). Without an understanding of how the informal setting can help to position a learner, it would be difficult to see the full picture of the learner in all contexts. While Bricker and Bell (2014) provide an exemplary case of positioning and self-identity, further research on this topic, and how it connects to other aspects of understanding the learner in informal learning environments, is greatly needed. 


\section{Understanding the Museum Setting}

Just as understanding the role of the learner was an important focus in the literature, so too was understanding the ways in which the museum setting affected learning. This theme in the literature can be categorized under the following topics: labeling, aesthetics, and interaction in exhibit design; the role of the facilitator; skill development from exhibit experiences; evaluation and assessment of exhibits; and makerspaces and the maker movement. Each of these areas will be explored further in the sections that follow.

\section{Exhibit Design: Labeling, Aesthetics, and Interaction}

Paris (1997) explains that "a hallmark of museum exhibits is their openendedness; visitors are free to approach, engage, and persist at their own pace" (p. 23). How an exhibit is designed can greatly influence the visitor experience and the learning that takes place, which indicates in part the effectiveness of the exhibit itself. Exhibit design focuses not just on what content knowledge is being transmitted to the visitor, but also how that knowledge is coming across, including everything from labeling and aesthetics to the kinds of interactions that visitors experience with the exhibit. When research is conducted in a museum, the exhibits often receive scrutiny because they are "the smallest unit of a visitor's experience and lend themselves to analysis" and yet "it is the cumulative or whole experience that makes a visit memorable" (Barriault \& Pearson, 2010, p. 104). Most of the informal science research addressing museum exhibits over the last decade has focused on two core areas of design: labeling and aesthetics, and type of interaction. 
How an exhibit is labeled can ultimately influence learning in a museum because labels have the "ability to structure and sustain visitors' interactions and interests in an exhibit" (Dudzinska-Przesmitzki \& Grenier, 2008, p. 18). The same can be said for the way in which the exhibit is presented visually. Gutwill (2008) explains that "offering visitors beautiful aesthetics, opportunities for creation, and intriguing representations often leads to active prolonged engagement" (p. 192). Yet poor labeling or aesthetics can be counterproductive. Labeling in particular can shift the ways in which visitors interact with a museum exhibit. Gutwill (2008) studied visitors to the Exploratorium in San Francisco and found that how a label was written impacted the level of inquiry visitors were able to achieve. "Although explanatory labels may satisfy visitors and even teach them science concepts, such labels may actually inhibit inquiry" (p. 192). Schauble, Leinhardt, and Martin (1997) elaborate further, explaining that "an effective label cannot be decided apart from concern for the gallery where the labels will appear, who is expected to read them, the function they are to serve, and the message they convey" (p. $5)$.

Changing the text of a label can elicit higher levels of learning as well. Bradburne, as discussed in Dudzinska-Przesmitzki and Grenier (2008), provides a telling example of a jewelry display box containing a variety of glasses. Changing the label on the display from "Glasses through the century" to "One of these glasses is fake" shifted the direction of learning and resulted in longer visitor attention and interest in the exhibit (p. 17). This example perfectly illustrates how labelling can influence a visitor's learning experience. Overall, labeling and aesthetics as well as type of interaction are critical to designing exhibits which engage the visitor, capture visitor interest, and lead to higher levels of 
learning. But these are not the only components of the museum experience that can influence visitor learning. The role of the facilitator is also critical to shaping visitor experiences (Sedzielarz, 2003) and is discussed in the following section.

\section{The Role of the Facilitator}

Facilitators exist in many forms in the museum setting. They can include museum educators, classroom teachers, parents, chaperones, volunteers, and even other students. Often, what a learner gets out of an informal learning experience depends largely on the facilitator, but for many museums, "funding constraints and staff reductions have left fewer experienced and knowledgeable full-time staff" (Castle, 2006, p. 131). As a result, many of the facilitators are often just volunteers or part-time staffers who don't often have the "incentive, time, or energy to pursue additional training and continuing development of their teaching practice" (Castle, 2006, p. 131). In order to develop desired traits and dispositions in visitors, the facilitators need to reflect those same dispositions. As Ritchhart (2007) explains, "if one of the dispositions educators want to develop in students is that of looking closely and noticing, educators must model it and provide time for it" (p. 144).

Critical thinking skills are one of the areas which museum research has shown to be a key focus, as will be discussed in a later section. How those skills develop has been linked to the ability of the facilitator. A study by Luke, Stein, Foutz, and Adams (2007) examined the success of a newly developed instrument for measuring critical thinking in art museums. Piloted by ten museums across the country, participants commented on the role of facilitators related to critical thinking, saying:

"what [the observation] revealed to me," said a Cincinnati Art Museum educator, "is how easy it is to set students up for success or failure when it 
comes to critical thinking...having a good facilitator is key to students developing their critical thinking." A Museum of Fine Arts, Boston, educator agreed, adding, "what this suggests is that there might be a great deal of critical thinking going on, but unless we ask the right questions or create the right environment to elicit it, we don't see it." (p. 133).

Research by Felton and Kuhn (2007) also demonstrated the importance of facilitators in their ability to lead visitors to "examine their understanding, revise their misconceptions, and build new knowledge on a stronger and more elaborate base of prior knowledge" (p. 102). Overall, development of critical thinking skills was more likely when facilitators were well informed and well trained.

Given the importance of facilitators to developing critical thinking skills, schools experience an added burden of finding appropriate chaperones for field trips, because these chaperones often function as stand-in educators and facilitators. One specific chaperone role cannot be assigned because the responsibility is incredibly complex. In an ethnographic study of 35 chaperones, Sedzielarz (2003) found that "each chaperone comes to the trip with his or her own expectations and understandings and that "assigning a single role to the chaperone is misunderstanding the complexity of this phenomenon" (p. 24). While the study was limited in that it only examined chaperones from self-guided field trips, the results reflected a need to further understand the role of the chaperone as a facilitator. Furthermore, Sedzielarz (2003) explains, "if we believe that school field trips are valuable learning experiences, we also need to regard all members of the field trip as learners" (p. 24). This includes the chaperones and any other volunteers who are taking part in the experience.

While chaperones of field trips often include parents of school-aged children, parents have their own unique role as a facilitator, especially for younger students. 
Researchers Crowley and Callanan (1998) conducted two studies in which they examined how parent-child interactions influenced collaborative learning in an informal learning environment. Their first study consisted of 49 child/parent pairs and 41 child only participants, and their second study consisted of 160 children/93 parents who prototyped an exhibit followed by 205 children/154 parents who experienced the redesigned exhibit. The results showed that the parent functioned as an important bridge between what the exhibit designer was trying to create and what the child experienced, and that "parent participation deepened children's engagement with the exhibit, both at the level of parents guiding activity and at the level of parents constructing explanations around that activity" (p. 15). Furthermore, Crowley and Callanan (1998) explain that "although there are times when children learn in relative isolation, much of what they learn about their world they learn in the context of parent-child interaction" (p. 17). This has important implications for learning because "in their dual roles as guide and interpreter, parents fundamentally shape children's scientific thinking during museum visits" (p. 17). Overall, the role of parent as facilitator is vitally important for content and skill development, especially in younger children, and is something museum educators should attend to closely in order to maximize the potential of the informal learning environment.

The role of a facilitator can mean a lot of different things even among museum educators. In a study conducted by Bailey (2003), 15 museum educators from science centers in Massachusetts were asked a series of questions relating to their perceived roles, identity, and practice within their field. The results suggested that educators often saw their role as facilitator as part of their personal identity rather than just a job (p. 180), even blurring the lines often between "activities they define as work and things they 
choose to do in their time off from their jobs" (p. 181). The educators also understood the importance of modeling desired dispositions, often themselves having "a highly developed sense of curiosity and eagerness to learn new things" (p. 193). Furthermore, Bailey (2003) explains that museum educators suggested they "do what they do because they believe in it, and they see a connection to the common good. They seek to engage, inspire, inform, and communicated their values and passions to members of their audience" (p. 194). This motivation often leads to financial sacrifices in exchange for these more altruistic purposes. However, for facilitators to truly be successful, they must have the right skills and attitude for their work, including: "knowledge about teaching and learning; talents for presentation and performance; program development and implementation skills; science content knowledge; project management capabilities; and a savvy about how their organization works and communicates" (p. 195). All of these perspectives and types of facilitators reveal how incredibly complex and intricate the role of the facilitator is in promoting visitor learning as well as visitor skill development.

\section{Skill Development from Exhibit Experience}

Content knowledge is often seen as a primary goal in formal educational environments, but that is not always the case in informal learning environments. In fact, much of the research shows that informal spaces pride themselves on being able to link content from the classroom with experiences and skills needed in the real world. A primary component of the mission and vision statement of many science museums and centers is to develop a public understanding of science, which is distinctly different from having scientific content knowledge. According to Storksdieck, Jones, Falk, and Alpert (2002), a public understanding of science aspires to "allow the public to learn about 
science, think about science, and then judge information they receive about science with sufficient background knowledge as to allow them to develop rational opinions and positions" (p. 14). Crowley and Callanan's (1998) study of child-parent collaborative learning highlighted the importance of museums in allowing children to "exercise more general competencies in forming expectations, evaluating evidence, and constructing explanations" (p. 12). The researchers go on to explain how this influences the ways in which exhibits are designed. "At its core, science is a way of making sense of the world. It is a way of building up new theories to explain existing evidence and a way of seeking out new evidence to revise existing theories. Successful exhibits are those that support opportunities for children to think through the process of coordinating theory and evidence" (p. 12).

Bricker and Bell's (2014) longitudinal study of Brenda and Stella also emphasized the importance of scientific practices over content knowledge. Through their discussion with Brenda and Stella about a perfume kit that Brenda played with at home, the researchers recognized that content was not an important goal but rather, the focus was on the practice of doing science. They explain:

The scientific content was not the focus of Brenda and Stella's Perfumeryrelated discourse, however. Brenda and Stella were focused instead on the practices involved in making perfumes, as well as the uses of the perfumes in their lives (e.g., gifting perfumes to friends and relatives, spraying perfume into the air in their home). We argue that the material practices and the learning involved in their coordination and implementation are important outcomes in their own right with respect to STEM-related learning (p. 272).

Brenda's actions at home in informal learning settings demonstrated many scientific practices that are valued in the formal education field of science, including "(a) control for contamination with apparatus, (b) careful measurement of materials during 
implementation of protocols, (c) tracking and labeling of samples, and (d) systematic journaling of results" (p. 271). This demonstrates the ability for informal experiences to function as a bridge between the informal learning environment and the formal classroom. In fact, informal environments often initiate interest in a particular hobby, such as perfumery in Brenda's case, and can later lead to an interest in formal science because of that initial interest developed outside of the school setting (Azevedo, 2013; Bricker \& Bell, 2014).

Places like museums and science centers provide the opportunity for students to develop other important life skills that will also benefit them in the classroom. These skills are referred to by Root-Bernstein and Root-Bernstein (2005) as creative thinking or imaginative tools that are needed to generate ideas, and their function can vary depending on the setting. These tools include: observing, imaging, abstracting, recognizing and forming patterns, analogizing, body thinking, empathizing, dimensional thinking, modeling, playing, transforming, and synthesizing (p. 3). While some of these skills or tools are embedded in the formal classroom, they are not typically the primary learning objectives. Yet development of these skills in the informal environment can support the learning objectives in the classroom. In a three year quasi-experimental study conducted by Burchenal and Grohe (2007), the researchers examined the impact of school partnership programs (SPP) in which students visited the same museum multiple times over the course of the year. The study consisted of an experimental group of fourth and fifth graders who were enrolled at two SPP schools, and a control group of fourth and fifth graders enrolled at two non-SPP schools who shared similar test scores, race/SES demographics, and instructional philosophies. The researchers found that the multiple 
visits made a difference in non-content based skills such as how to critically analyze and interpret an art exhibit, explaining that "by helping students develop looking skills in sequential, multiple-visit programs, museums are in fact still supporting school learning by focusing on transferable skills rather than content" (p. 112-113). In this case, the transferable skills had to do with critical analysis and interpretation, but many studies focus on critical thinking and scientific reasoning as skills that can be developed and transferred from the museum setting into the classroom.

Critical thinking skills are often the center of attention in both the formal and informal environments, and are therefore central in the design and development of museum exhibits, because the development of these skills allows us "the means to be reflective, thoughtful, and creative citizens" (Luke, Stein, Foutz, \& Adams, 2007, p. 123). Yet, developing these skills is not an easy task. Research by Felton and Kuhn (2007) suggests that "critical thinking requires considerable effort, and visitors will only engage in the complex work of critically evaluating their knowledge if they believe that it is worth the effort" (p. 103). As a result, it is important that exhibits be engaging and developed in such a way that visitors have extended time to interact and experience that exhibit. Often, a quick reaction to this problem is to make the museum exhibit more interactive. Unfortunately, exhibit designers seem to have equated reactivity (i.e. the pushing of a button) with interactivity, which leads to exhibits that provides visitors with an initial interest but not much engagement after that. In a 2007 study by Ritchhart, the researcher examined the importance of not just engaging visitors but also developing within them a disposition to be good critical thinkers. He explained that ability alone is not sufficient; rather, "one must also have the inclination to use that ability along with the 
awareness of opportunities for its use" (p. 138). Wanting to develop this disposition should not be surprising, but museums often forget that critical thinking skills alone are not sufficient, even though, as Ritchhart (2007) explains, "We readily recognize the dispositional nature of curiosity, acknowledging that having a set of skills alone, such as being able to ask questions or pose wonderings, does not make someone curious. It is an individual's awareness of occasions for applying those skills and being inclined and motivated to use his or her abilities that leads us to call a person curious" (p. 138).

Through his study of three museums in which tours were observed for a series of cultural forces that shape group learning and dispositions, Ritchhart (2007) found that visitors were only able to develop a disposition toward critical thinking when they had the opportunity to fully engage with the exhibit. He explains that "without time to engage properly with an object or idea, an opportunity for thinking can feel hollow. It is only through extended inquiry that conjectures can be made, perspectives can be examined, theories weighed, and new understandings developed" (p. 143). Ritchhart's study emphasizes the importance for museums to not just focus on developing critical thinking skills but to also focus on developing dispositions. Yet, measuring the public's understanding of science or the development of life skills such as critical thinking is incredibly difficult and is seen as a major challenge for informal education. Downey, Delamatre, and Jones (2007) explain that "in this age of standardized testing, museum educators feel the pressure of accountability and assessment. Museum educators believe that their programs have positive effects on student achievement, and ambitious educational aims are common among museum-school programs: increasing students' critical thinking skills, scientific-thinking skills, communication skills, creativity, and 
problem-solving abilities" (p. 175). The issues of evaluation and assessment in museum settings are a real barrier and will be discussed further in the following section.

\section{Evaluation and Assessment of Learning}

Evaluation and assessment of learning is very different in the museum setting than in the formal setting, though it is of critical importance given that museums often rely on public and government funding and are therefore responsible for meeting the needs of various stakeholders, who want to know everything from how effective the programs are being implemented to what impact the museum is having on the community (Stone, 2008; Persson, 2000; Rennie \& Johnson, 2007; Barriault \& Pearson, 2010). However, few effective tools have been developed to measure the learning objectives of museum settings, and so traditional academic measures are often used (Hofstein \& Rosenfeld, 1996). This creates a major problem because, as Martin and Toon (2003) suggest, "for many museum researchers, measuring informal learning unobtrusively is a problem because most of our measures, derived from academic settings, fundamentally change the nature of the informal experience" (p. 15). Because of the free-choice learning environment, assessing learning in museums "cannot be accomplished with the same methods that are used in formal instructional settings where participation is mandatory and the learning objectives are explicit and strongly focused on cognitive gains" (Barriault \& Pearson, 2010, p. 91). Museum assessment should take into account the other aspects of learning, including the emotional and affective skills that are developed and the personal nature of the experience, which is unique to each visitor. Additionally, stakeholders are often looking for quantitative results from museum learning, but museum research tends to focus on qualitative measures such as observations, interviews, 
and basic head counts and time spent at specific exhibits to measure the level of visitor engagement. Crowley and Callanan (1998) explain that "the operational problem is that it is extraordinarily easy to shadow visitors or measure time-on-task, and it is extraordinarily difficult to construct reliable measures of curiosity, fun, or interest" (p. 14). As a result, museum educators often rely on intuition and informal feedback to know whether their museum programming is having a positive influence on student learning (Downey, Delamatre, \& Jones, 2007). So, if the informal science research literature recognizes assessment and evaluation as an area which needs some attention, then why has it not been addressed? The answer, not surprisingly, comes down to time and resources.

Just as schools struggle with appropriate time and resources, including financial resources, museums experience the same struggles. Research literature shows that there are two primary reasons for the lack of evaluation and assessment by museums. First, the museum staff does not have the time nor staffing to conduct evaluations, and they are often not trained to do so either. As Worts (2006) explains, "there are countless indicators that can be used to provide insight into whether visitors actually engage in reflective and meaningful ways with museum programs, but all too frequently museums do not dedicate the time or resources to collect this essential feedback in order to improve their methods of understanding and engaging audiences" (p. 45). Second, museums often do not have the financial resources, nor are they eligible to receive funds, to conduct the kind of rigorous research that is needed in the informal learning environment. As Barriault and Pearson (2010) clarify, "most methodologies used by researchers and external evaluators require substantial financial and human resources as well as expertise in investigation 
methods....Resource demands of research remain a difficult hurdle in conducting research on the visitor learning experience and exhibit evaluation" (p. 92). As funding continues to be more tightly linked with quantitative results, schools and museums both must find a solution to provide appropriate assessment and evaluation for their programs. Having practical research tools that allow the museum staff to conduct evaluations and assessment is critical for the future of informal learning (Barriault \& Pearson, 2010). As Downey, Delamatre, and Jones (2007) explain, "regular assessment is key to maintaining relationships with program stakeholders outside of the museum such as funders, school districts, teachers, and parents" (p. 181). Without regular assessment and evaluation that can be conducted in a way that works for a majority of museums who do not have the time, resources, or financial support to participate in traditional methods of evaluation, informal learning educators will continue to rely on intuition and informal feedback to make important decisions regarding programming and development.

\section{Summary}

In summary, while many museum studies focus on how visitors think and interact within a museum setting from a sociocultural theoretical standpoint, this study's emphasis on the hands-on learning and engagement that occurs in a children's museum makerspace exhibit makes it better suited for a constructivist theoretical framework. The literature tells us that understanding the learner is critical to understanding the factors that influence visitor engagement. The prior knowledge that a visitor brings with them influences his agenda and motivation toward each exhibit, and making sense of new knowledge can be elusive or might occur outside the museum setting. However, positioning the visitor to develop a strong sense of identity can help bridge the formal and 
informal learning environments and increase engagement in the museum setting. Even more important for museum engagement might be the museum setting itself. How the exhibit is designed and labelled, what role the facilitator plays, and what skills the visitor can develop from an exhibit all influence the level of engagement that can be attained. Because of all of these seemingly intangible characteristics of museums, evaluation and assessment can be difficult, especially given that many of the measurement tools in the informal setting were developed within the context of the formal learning environment. Therefore, the design and methodology of the study must take into account all of these factors when trying to measure levels of engagement. 


\section{CHAPTER III METHODOLOGY \\ Statement of the Problem}

Many challenges exist in finding ways to measure informal learning environments in ways that are not anecdotal in nature or that examine engagement by intuition or informal feedback. Children's museums in particular are prone to this, adding new exhibits and programs often based simply on visitor or parental feedback, or based on trending programs in other museums (Family Learning Forum, 2013). Makerspaces are a perfect example of this because their addition to museums has been a result of the trending maker movement. As the maker movement continues to grow, it is important to identify whether there are useful benefits for including makerspaces, which tend to be a resource- and time-intensive exhibit in Children's Museums (Halverson \& Sheridan, 2014). Makerspaces in museums such as the Children's Museum of Pittsburgh and the New York Hall of Science have shown how the maker movement can be accessible to young people and families, and their "focus on maker culture is a natural extension of a long-standing emphasis on learning-by-doing in art and science" (Halverson \& Sheridan, 2014, p. 499). However, few studies of their impact have been published. Given the relatively new inclusion of makerspaces in Children's Museums and the lack of research that currently exists, a number of questions arise. Understanding the level of engagement within makerspaces will help us to better understand how learning might be occurring in 
museum makerspaces and provide guidance for future exhibits to be effective without the need for programmatic and staff-intensive scaffolding. Additionally, understanding how demographic characteristics influence engagement outcomes will provide insight into the need for potential interventions or models for success. Therefore, the purpose of this concurrent mixed methods study was to better understand engagement and learning by converging both quantitative and qualitative data. In the study, an observation protocol developed by Barriault and Pearson (2010) known as the Visitor Based Learning Framework (VBLF) was used to measure the engagement levels of children in a museum makerspace. At the same time, field notes were collected to explore the context in which this engagement takes place. The following research questions were used to guide this study:

1. At what levels, based on the VBLF, do children engage with the makerspace exhibit in the museum?

2. What relationships exist between engagement levels and age and gender?

3. How do makerspace engagement levels compare to average engagement levels of traditional museum exhibits?

\section{Research Design}

Because of its ability to allow for an expansion of understanding from one method to another, and because it presents the ability to converge or confirm findings from different data sources (Creswell, 2003), a mixed-method approach is the best method for answering these research questions and was chosen for this study. This study used a mixed methods design to examine children's engagement levels and science learning when they participate in a makerspace exhibit. Mixed-methods techniques incorporate 
components of both qualitative and quantitative data to offer a variety of perspectives on a research study and are becoming more prevalent. As Creswell (2003) explains, "with the development and perceived legitimacy of both qualitative and quantitative research in the social and human sciences, mixed methods research, employing the data collection associated with both forms of data, is expanding" (p. 208). In the case of this study, mixed-methods is necessary in order to provide context to the quantitative data collected from the observation protocol. This design, known as a concurrent triangulation strategy, focuses on collecting both qualitative and quantitative data simultaneously and then using the different methods in order to confirm, cross-validate, or corroborate the findings (Cresswell, 2003, p. 217).

Table 1

A Summary of the Research Study Design

\begin{tabular}{|c|c|c|}
\hline Research Question & Data Sources & Data Analysis \\
\hline \multirow[t]{2}{*}{$\begin{array}{l}\text { 1. At what levels do children } \\
\text { engage with the makerspace } \\
\text { exhibit in the museum? }\end{array}$} & Observation Protocol & $\begin{array}{l}\text { Descriptive analysis (means, } \\
\text { standard deviations, } \\
\text { frequencies, percentages) }\end{array}$ \\
\hline & Field Notes & Thematic/Conceptual coding \\
\hline \multirow[t]{2}{*}{$\begin{array}{l}\text { 2. What relationships exist } \\
\text { between engagement levels } \\
\text { and age and gender? }\end{array}$} & Observation Protocol & $\begin{array}{l}\text { Correlation and statistical } \\
\text { significance (cross-tabs } \\
\text { analysis) }\end{array}$ \\
\hline & Field Notes & Thematic/Conceptual coding \\
\hline $\begin{array}{l}\text { 3. How do makerspace } \\
\text { engagement levels compare to } \\
\text { average engagement levels of } \\
\text { traditional museum exhibits? }\end{array}$ & Observation Protocol & $\begin{array}{l}\text { Correlation and statistical } \\
\text { significance (chi-square } \\
\text { analysis) }\end{array}$ \\
\hline
\end{tabular}

Mixed-methods designs do present challenges because they involve extensive data collection, are time-intensive, and require the researcher to be familiar with both 
qualitative and quantitative forms of research; however, the results can be very rewarding and provide valuable results (Cresswell, 2003). A summary of the study design can be seen in Table 1 above.

This study uses a naturalistic observational design, which is defined by Vogt, Gardner, and Haeffele (2012) as one that "stresses investigating individual and social life in its natural settings, without intervention by a researcher (p. 345). Using an observational design for this study is important because as Merriam (1998) explains, "Observation is the best technique to use when an activity, event, or situation can be observed firsthand, when a fresh perspective is desired, or when participants are not able or willing to discuss the topic under study" (p. 96). Observations can serve as a primary source of data in qualitative research and can be used to help triangulate emerging findings. This kind of design is important because it gathers evidence from "events, situations, settings, practices, and other social phenomena as they occur" in order to provide a firsthand account of the observed phenomenon (Vogt, Gardner, \& Haeffele, 2012, p. 67). Observation itself is a very natural process. In fact, we observe phenomena every day. However, not all observations are research-worthy. According to Kidder (1981), an observation can be used as a research tool if it “(1) serves a formulated research purpose, (2) is planned deliberately, (3) is recorded systematically, and (4) is subjected to checks and controls on validity and reliability" (p. 264). This study uses an observational protocol to fulfill the research purpose and objectives and to answer the research questions. A naturalistic observational design offers both advantages and limitations to the study. The major advantage is that the researcher can record information as it is revealed, uncovering unusual aspects while they are happening 
(Cresswell, 2003, p. 186). The major limitation for this study is that the potential exists for the researcher to be seen as intrusive. However, given the public setting and the frequency with which other staff members and volunteers came in and out of the research area, the intrusiveness was limited.

This study took an overt naturalistic observational design approach in which the researcher role was explicitly stated but the researcher remained in the background during observations (Vogt, Gardner, \& Haeffele, 2012). Quantitative data were generated from the observation protocol components and were used to answer research questions one, two, and three. Qualitative data was generated from extensive field notes, which were recorded for each participant. The focus of these field notes was the learning and behaviors that were occurring while the participants were in the makerspace. This qualitative data was used to contextualize the quantitative results for the research questions. The process of recording observational field notes can be time consuming and difficult. No one can observe everything, but elements that are likely to be included in an observational design include the physical setting, participant information, activities and interactions, conversations that occur, subtle factors that influence the participant, and the researcher's own behavior and comments (Merriam, 1998, p. 97-98). For this study, the process for recording observational field notes for each participant began with a review of the observational framework that was being used for the study. The field note process began by describing the visitor age and gender as well as distinguishing factors (i.e. red shirt and blond hair) to keep the participant separate from other visitors to the space during that time. Following the initial description of the participant and space, continual observational notes were taken during which any dialogue or activity was recorded. 
Because a computer was used, it was possible to type field notes while simultaneously conducting visual and auditory observations. After the participant left the space, the field notes were concluded. Finally, after the day of observations, the researcher reviewed all field notes for the day and updated or filled in missing information based on memory recollection.

\section{Site Selection}

The study was designed to take place in a museum makerspace. The study required a space that was open and accessible to all visitors attending the museum and that maintained the fundamentals of the maker movement. A local children's museum was chosen to fulfill these needs because it met these criteria. Other regional museums were ruled out because they lacked a makerspace or the space was not available to all

museum visitors, or the makerspace existed outside the context of a museum setting. The study also required extensive observational data collection over the course of months, so proximity was important. The chosen site was a well-established children's museum which had recently opened a makerspace exhibit. The museum itself was designed for toddlers through pre-teens with a strong focus on open-ended learning, hands-on play, exploration, and discovery. The makerspace was designed to accommodate open exploration as well as facilitator-led activities. The makerspace included four workbenches with tools as seen in Figure 1 below. 


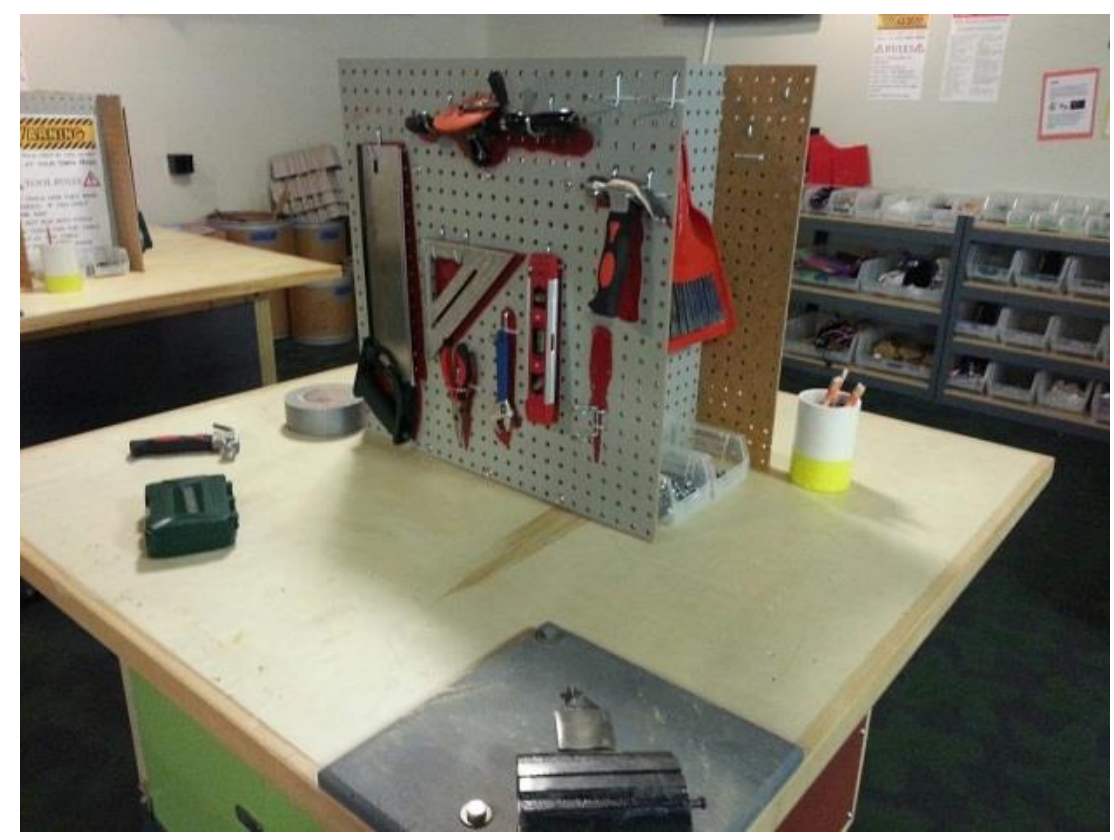

Figure 1. Makerspace workbench at a children's museum

The tools on the workbenches were moveable so that the benches could become flat surfaces on which to conduct experiments or other activities. In addition to the benches, the makerspace contained a section of materials sorted in bins, including items that ranged from pipe cleaners to old electronic parts to small and large pieces of wood. Finally, hot glue guns were available at a separate station away from the general work benches. Because of the equipment and materials used in the space, all visitors were required to wear safety goggles. Because the museum has an annual attendance of over 130,000 visitors, this allowed for sufficient data collection over the course of two months.

In the makerspace that served as the site for this study, there were specific themes during the week, with open maker days on the weekend. During data collection, five themes were observed: pollinators, hydraulics, open lab, squishy circuit, and stop-motion animation. All of the themes were organized in the same manner except the open lab, 
which allowed visitors to come in and build/design/create without any prompts or focused activities. The typical organization started with an introductory activity to demonstrate the concept. That was followed by a second activity that involved the concept and some component of making. Finally, the visitors were given the opportunity to make their own creation as it related to the theme for the day. For example, during the squishy circuit week, visitors started with the introductory activity of learning about the difference between insulators and conductors. To do this, visitors approached the first work bench where a bunch of materials were laid out and they had to test different materials to see if they could make the light bulb light up. Once they had an understanding of insulators and conductors, they moved to the second station where they learned about conductive and inductive dough. They got to play with the dough and try it out in different circuits to see if they could tell which one was the conducting dough and which one was the insulating dough. Finally, they moved to the third station where they could use the dough to make their own creation with the goal of using the dough and LED lights to create something that would light up. The most popular creation was building a creature with eyes that lit up.

\section{Participant Selection}

Participants of this study included child-parent/guardian visitor pairs who choose to interact with the makerspace exhibit at a local children's museum. Though children are required to have a parent or adult with them to interact with the exhibit, for the purpose of this study the "participant" was defined as the child visitor to the space. Therefore, engagement levels relate to the child, though parent-child interactions were prominent in the field notes. Consistent with the typical visitor age range for the museum, 
most participants were between the ages of 3 and 10. Age was determined with the help of the facilitator of the space. As visitors entered the makerspace, the facilitator asked each visitor how old he/she was before explaining the instructions for that day's activities. That allowed the researcher to record the exact age of the participant in the field notes. Data collection occurred in the summer, when the makerspace exhibit was open every Monday through Saturday, from 10 a.m. to 1 p.m. A total of 17 days of observations were conducted, for a total of 32 of the possible 51 hours, between June $18^{\text {th }}$ and July $24^{\text {th }}$. Because of the intensive nature of data collection and observation for this instrument, a maximum of two visitors were observed at a time following the observation protocol. Participants were selected based on when they enter the exhibit. The first two visitors to enter the exhibit were observed. After one of those visitors left the exhibit, the next visitor to enter was observed as the next participant, and so on. For busier days, this method resulted in observations of a little more than $50 \%$ of the visitors to that space during the time in which observations were occurring. On slower days when visitors were entering the space and leaving before additional visitors came, it was possible to observe most if not all of the visitors. In total, 90 visitors were observed out of a possible 153 visitors (59\%) to the space. Of the 90 participants, $51 \%$ were male and $49 \%$ were female. The majority of visitors (62.2\%) fell in the child age range of 6-10 years old, followed by the young child age range of 0-6 years old (34.4\%) and pre-teens in age range 11-13 $(3.3 \%)$.

\section{Observations}

The initial observations on the first day of a new theme included extra attention to the process of the activities being conducted and the responses of the visitors to those 
activities. On following days, observations were tailored more specifically to how the visitors were interacting with the exhibit activities. During the data collection process, the researcher had a copy of the observational framework in order to best align the engagement levels being observed with those that had previously been identified in the framework (which can be found in Table 2 on the following pages). As visitors participated in the makerspace activities, the researcher recorded any instances of engagement behaviors that aligned with the protocol. Once a visitor exhibited that engagement behavior, it did not need to be recorded again on the instrument, though it was recorded in the field notes. Once visitors left the makerspace, the researcher stopped recording data for the protocol as well as field notes. Each participant had a completed observation protocol as well as detailed field notes by the end of their visit to the space. The researcher was able to note which behaviors were unique and which were typical for the activity, which allowed further refinement of the field notes. Because of the nature of the exhibit and of the observation protocol, all activity from a visitor once they entered the space was observed until they left the space.

Field notes marked changes in activity and any important dialogue or body language that occurred during specific activities over the course of the observation. Essentially, from the moment the participant entered the space, the researcher began recording any dialogue or body language that was occurring in relation to the activity. The researcher also tried to note differences in the ways that facilitators presented the activities to the visitors, and any other interactions with other visitors or adults that occurred during the participant's time in the makerspace. As the participant worked on his/her activities and projects, field notes were recorded every time a new task was 
started or a shift in activity occurred. Observations that aligned with the protocol were also recorded in the field notes. The only information that was purposefully left out of the field notes related to specific details about the activities that were recorded for the first visitor of that activity and then only recorded again when there was a change. For example, field notes for visitor 30 describe the squishy circuit activities in detail but the rest of the visitors who completed the squishy circuit activities only had enough detail to refer back to the first description unless something changed in the process of conducting the activity.

\section{Instrument}

The observation protocol instrument used in this study was a Visitor Based Learning Framework developed by Barriault (1999) which categorizes students' stages of learning as they engage with a museum exhibit. For this study, the focus was specifically centered on the makerspace exhibit. The development of the instrument, the observational behaviors that occur, and the reliability and validity of the instrument are discussed below.

\section{Development}

Initially developed in the late 1990s for use at Science North, a science center in Ontario, Canada, the Visitor Based Learning Framework (VBLF) has been used in science centers for more than a decade and is seen as "a practical tool for science center staff to easily understand the impact the exhibits have on visitors' learning behaviors" (Barriault \& Pearson, 2010, p. 98). Observational and interview data from science centers were used to empirically develop the levels of engagement and organize them in a way that reflected not only increased engagement but also increased depth of learning. Once 
developed, the framework emerged as "an effective assessment tool for science center practitioners to evaluate the potential impact of exhibits on visitor learning" (Barriault, 2014, p. 24). The instrument was then used by Barriault and her colleagues over many years and continued to provide evidence of the learning impact of museum exhibits, which eventually led to its transition into a model to help practitioners assess and improve the impact of their exhibits by identifying areas where intervention may result in higher breakthrough levels of engagement. As a result, the instrument has been used by many other researchers and practitioners to evaluate learning impacts of exhibits and to train staff to identify learning behaviors (Harkins, 2011; Visscher \& Morrissey, 2010). Most uses of this framework have taken place in a science center setting, though a modified version of the instrument was developed for use in zoos and aquaria (Barriault, 2014). However, this study uses the framework in a children's museum exhibit setting, which is expected to be similar to most science center exhibits in its focus on hands-on, authentic learning experiences.

\section{Observational Behaviors}

The observation framework includes seven learning behaviors grouped into three categories reflecting increasing engagement. These categories are: initiation behaviors, transition behaviors, and breakthrough behaviors. Within the category of initiation behaviors, the researcher is looking for the behaviors of doing or of watching others engaging. These behaviors indicate that the visitor is taking preliminary steps toward meaningful engagement. As Barriault (2008) explains, these behaviors "enable visitors to 'test the waters' with minimal personal risk and provide an entry point into further learning opportunities offered by the exhibit" (p. 17). At the transition level, the research 
is looking for the behaviors of repeating the activity or demonstrating a positive emotional response. These behaviors demonstrate increased engagement and show that the visitor has an increased level of comfort with the exhibit, which leads to more commitment and motivation toward learning (Barriault, 2008). Finally, the researcher is looking for behaviors of referring to past experience, seeking and sharing information, or being engaged and involved (i.e. testing variables, making comparisons, using information gained from the activity), all of which exhibit breakthrough behaviors. At this level, real learning can occur because the visitor has established a personal level of comfort allowing a free exchange of ideas and is demonstrating an acknowledgement of "the relevance of the activity, and the learning gained form the activity, to the individual's everyday life" (Barriault, 2008, p. 18).

These observations are then quantified into what is described by Barriault (1999) as a Visitor Engagement Profile (VEP), in which the y-axis represents the percentage of visitors who engage in the learning behaviors on the $\mathrm{x}$-axis. It is important to note that in all of these VEPs, the initiation behavior is at $100 \%$ because of the fact that only those who approach the exhibit are counted or observed. As Barriault (2008) explains, "The VEP is not intended to be a measure of the attracting power because of the challenge of assessing the reasons why particular visitors did not approach the exhibit. Instead, the VEP focuses our attention on the learning behaviors demonstrated by visitors once they have made the commitment to engage with the exhibit" (p. 20). While an ideal curve depends on the type of exhibit being assessed, Barriault (2008) explains that exhibits designed to involve visitors in hypothesis testing such as the makerspace should produce a VEP with at least $50 \%$ of visitors demonstrating breakthrough behaviors. 
Table 2

The Visitor Based Learning Framework from Barriault and Pearson (2010)

Engagement Levels and

Learning Behaviors

Types of Activity

Initiation

1. Doing the activity

- in passing, not done completely;

- doing the activity somewhat completely;

- doing the activity completely without further exploration or testing variables.

2. Spending time watching others engaging in activity or observing the exhibit

- looking at the exhibit working, or someone doing the activity;

- watching the exhibit or person using the exhibit with expressed interest in the activity;

- interested in learning outcome or in learning the activity; visitor does the activity after observing.

Transition

3. Repeating the activity

4. Expressing positive emotional response in reaction to engaging in activity
- doing the activity two to three times to attain desired outcome, to master exhibit's function;

- enjoyment of the outcome;

- changing the variables once looking for a difference in outcome, becoming engaged and involved.

- $\quad$ smiling, pleased with exhibit;

- stronger signs of enjoyment such as laughter, verbal reference to enjoyment;

- obvious signs of eagerness to participate, excited disposition.

\section{Breakthrough}

5. Referring to past experiences while engaging in the activity

6. Seeking and sharing information
- $\quad$ reference to past experience with exhibit or science center;

- $\quad$ simple reference to comparable experience in visitor's life;

- reference to comparable experience in their life as well as making comparisons and deductions based on observations of similarities and differences.

- calling someone over to look at exhibit, or to ask them to explain an exhibit, asking questions to staff or family member without lengthy discussion or exploration of topic;

- $\quad$ reading signage, having conversations about exhibit and related science with staff or family member/friend;

- $\quad$ sharing experience with others by explaining the exhibit to them, giving them details about gained information and observations; discussions with staff or family member/friend.

7. Engaged and involved; testing variables, making comparisons, using information gained from activity
- $\quad$ engaging in inquisitive behavior, exploratory actions such as repeating the activity several times, reading signage, asking questions, remaining on task for 2-3 minutes;

- concentration and motivation are obvious; doing the activity as a means to an end, or meeting a challenge; length of interaction is significant, 3-5 minutes; outcome of result of activity is important;

- $\quad$ experimenting, testing different variables, looking for different outcomes; engages in discussion with others about the various outcomes; experience "flow;" involved in the activity for long period of time, i.e. more than 5 minutes. 
The Visitor Based Learning Framework from Barriault and Pearson (2010) can be found in Table 2 above and shows the framework in detail, highlighting which activities represent each engagement level and learning behavior.

\section{Validity and Reliability}

Admittedly one of the areas of weakness of this framework is that the instrument has not been tested for validity and reliability. However, its continued and expanded use in the field of informal learning by both researchers and museum practitioners gives it increased credibility. This credibility establishes the instrument's face validity, which has been shown from the repeated use of the instrument by museum educators and researchers in a variety of settings over the last decade. Additionally, empirical data were used to construct the learning behavior levels of the framework. In describing the instrument's validity and reliability, Barriault explained:

It is important to note that the learning behaviors and engagement levels in the instrument are a proxy for learning and an indication of engagement. Together, they serve as an assessment tool that measures the potential learning impact of the exhibits being observed. In other words, the occurrence of learning behaviors indicate that the conditions and opportunities presented by the exhibit are conducive to engaging visitors in a learning experience (C. Barriault, personal communication, April 28, 2015).

In this study, internal validity and reliability were addressed. According to Merriam (1998), "internal validity deals with the question of how research findings match reality" (Merriam, 1998, p. 201). For this study, internal validity was addressed through triangulation and long-term observation. Field notes helped with triangulation by providing contextualization of the observational data. Construct validity could also be established in this study given that the instrument 
was aligned with empirical data on visitor engagement from previous studies (Barriault and Pearson, 2010). The field notes and observations conducted in this study demonstrated that engagement indicators and learning behaviors were consistent with those outlined in the Visitor Based Learning Framework. This consistency helps to establish construct validity through the use of convergent data analysis. Negative case analysis was used to ensure proper coding of data, which also helped to support construct validity.

Reliability generally refers to whether or not the results can be duplicated, but Merriam (1998) points out that, "reliability is problematic in the social sciences simply because human behavior is never static" (p. 205). Rather than think of reliability as the ability to duplicate the experimental results, instead more focus should be placed on the consistency of the results obtained by the data within the study (Lincoln and Guba, 1985). Trustworthiness helps with intra-rater reliability because of its focus on consistency in coding and data analysis. Repeated observations of the same phenomenon helped to increase the validity of the findings by allowing the researcher to continually re-evaluate engagement levels and notes of engagement throughout the data collection period to ensure consistency in applying behaviors to the participants. Observational codes were reviewed after every day of data collection. At the end of each week, codes were compared to the field notes to look for missing information or incorrect attribution of coding constructs, and then data were adjusted accordingly. This analysis was conducted for both the observational learning behavior codes as well as the 
emergent coding of the data. This consistency in labeling and attention to detail increases the trustworthiness of the study.

\section{Data Collection}

Two methods of data collection were used for this study. The first was an observational engagement framework developed by Barriault (1999). The second method was detailed field notes collected during the observations. Data collection occurred during the operating hours of the museum over an extended period of time in the summer. The researcher conducted all observations. Because the makerspace exhibit had limited hours and days in which it was open to the public, a variety of times and days were observed in an effort to have as accurate a sample as possible. For example, weekday observations often only include visitors who are not in summer school or camps, whereas Saturday observations might see more school-aged children. A total of 17 days of observations were conducted, for a total of 32 of the possible 51 hours, between June $18^{\text {th }}$ and July $24^{\text {th }}$. During that period of time, 90 visitors were observed out of a possible 153 visitors $(59 \%)$ to the space. For each of these visitors, an observation protocol was completed alongside detailed field notes of that participant's visit to the makerspace exhibit.

\section{Data Analysis}

The observation engagement data was compiled in Excel and then transferred to the Statistical Program for the Social Sciences (SPSS) data system for analysis. In order to answer the research questions posed in the study, descriptive data analyses such as means, standard deviations, frequencies, and percentages were conducted. Additionally, statistics such as two-way contingency table analysis using crosstabs and chi-square 
analysis were run to determine correlations and statistical significance. These statistical tests were chosen because they follow nonparametric procedures. The crosstabs analysis was used to measure whether there was statistical significance between variables (i.e. initiation behavior, transition behavior, breakthrough behavior, gender, age group). The chi-square analysis was used to measure whether the proportion of individuals who fell into the engagement categories was equal to the hypothesized values, which are based on the average results from exhibits in which the observation protocol was used.

Field notes were collected, and direct dialogue was recorded when possible. The notes were reviewed and coded according to themes within each engagement level that emerged over the course of the observations. Initial coding themes focused on patterns of similarities and differences between male and female participants as well as young child and child participants. Additionally, because five different activities took place over the course of the data collection period, the researcher looked for themes within each of the activities. Field notes were also coded for specific examples and dialogue that aligned with the framework itself. Finally, themes emerged regarding facilitator/participant interactions as well as parent/child interactions and were coded thusly. Observer comments were interwoven with the field notes. Overall, a constant comparative method of data analysis was used, which focused on the "continuous comparison of incidents, respondents' remarks, and so on, with each other" (Merriam, 1998, p. 179). The data analysis process occurred through three main elements. First, the data were sectioned into separate ideas or codes that each stood alone. For an example of a coded visitor activity, see Appendix B. Second, the coded data was separated into emergent categories, which can be found in Appendix C. These were categories that were seen in most of the data or 
that had heavy emotional or factual influence, especially related to the quantitative data. Finally, negative case analysis was used, where alternative interpretations of the data were considered by testing the categories against new data (Lincoln \& Guba, 1985). This process was not linear but rather recursive, meaning that as new data were generated, coding was compared and renamed or regrouped as necessary, a process known as bridging the data (Lincoln \& Guba, 1985). For this study, coding was done by hand.

\section{Ethical Considerations}

Permission to use the space for research was obtained from the director of the museum by making a request through e-mail to conduct the study, and signage was posted during the study to indicate that observations were taking place. The appropriate ethical procedures were followed for this mixed methods research design study. All ethical procedures were outlined in the IRB protocol and were followed in the dissemination and data collection procedures of the observation protocol and field notes.

In addition, Gutwill's $(2002,2003)$ method of implied consent was used for this study. The benefit of using implied consent through Gutwill's posted-sign method is that it maintains anonymity, is less disruptive to participants, and has been successfully used in museums across the United States for decades. Federal guidelines require that in order for visitors to grant or withhold consent, they first must know that they will be recorded (or in this case, observed), understand how the observation will be used, and know that they may decline being observed (U.S. Office for Human Research Protections, 2009; Department of Health, Education, and Welfare, 1979). Since the mid-1980s, museum researchers have used the posted-sign method, inferring that if a visitor enters an exhibit area being studied, then that visitor has given their consent to be recorded/observed 
(Tully \& Lucas, 1986; Lucas, McManus \& Thomas, 1986). In a study by Gutwill (2003) examining the effectiveness of the posted-sign method at gaining consent, 197 visitors out of $200(99 \%)$ knew they were being studied and understood that they were consenting to being videotaped. The three visitors who did not know they were being recorded indicated they were not bothered by it and would still enter the exhibit area anyway (Gutwill, 2003). This study took place at the Exploratorium in San Francisco, where the posted-sign method has been used effectively for implied consent for their broad audience of both children and adults.

For the purpose of this study, the researcher was observing participants in a museum makerspace exhibit. No verbal contact was made between the researcher and participants, and no identifying information was collected throughout the entire process. Therefore, participants remained completely anonymous to the researcher. Using a traditional method of informed consent would introduce unnecessary risk to the participant and could even been seen as intrusive and off-putting (Callanan, 2012). Furthermore, participants might "perform" for the researcher. Therefore, the use of implied consent through the posted-sign method was chosen because it made participants aware of the research and observations without intruding on their museum experience, and it provided a more natural environment for the observations to occur while still obtaining the consent of the participants. 


\section{CHAPTER IV \\ RESULTS, ANALYSIS, AND DISCUSSION \\ Results and Discussion}

In the paragraphs that follow, results for each research question are presented, an analysis of data is conducted, and a discussion of the data in relation to the research question follows. Research question one explores engagement levels in the makerspace and is subdivided into the following categories: overall engagement, engagement by activity, and influences on engagement. Research question two explores the relationships between engagement level and age and between engagement level and gender. Finally, research question three explores how engagement levels in the makerspace compare to engagement levels in a traditional museum based on previous research.

\section{Research Question One}

The first research question was focused on answering at what levels children engage with the makerspace exhibit in the museum. Data from the observation protocol were used to create the Visitor Engagement Profiles (VEPs) and descriptive analyses were run using means, standard deviations, frequencies, and percentages. The field notes were used to contextualize the quantitative data.

\section{Overall Engagement Level}

As explained earlier, all visitors reached the initiation level of engagement because it was measured based on who entered the space. As a result, the frequency level 
for initiation was $100 \%$. Overall transition behavior levels were high in the makerspace, with a frequency of $65.8 \%$. Breakthrough behavior levels overall were also high, with a frequency of 51.1\%. Table 3 demonstrates the frequency with which each behavior level was reached.

Table 3

\begin{tabular}{|c|c|c|c|c|}
\hline Behavior Category & Frequency & Percent & Valid Percent & $\begin{array}{l}\text { Cumulative } \\
\text { Percent }\end{array}$ \\
\hline \multicolumn{5}{|l|}{ Initiation } \\
\hline Does not exhibit & 0 & 0 & 0 & 0 \\
\hline Exhibits & 90 & 100.0 & 100.0 & 100.0 \\
\hline Total & 90 & 100.0 & 100.0 & \\
\hline \multicolumn{5}{|l|}{ Transition } \\
\hline Does not exhibit & 31 & 34.4 & 34.4 & 34.4 \\
\hline Exhibits & 59 & 65.8 & 65.8 & 100.0 \\
\hline Total & 90 & 100.0 & 100.0 & \\
\hline \multicolumn{5}{|l|}{ Breakthrough } \\
\hline Does not exhibit & 44 & 48.9 & 48.9 & 48.9 \\
\hline Exhibits & 46 & 51.1 & 51.1 & 100.0 \\
\hline Total & 90 & 100.0 & 100.0 & \\
\hline
\end{tabular}

Engagement levels can also be broken down by each indicator within the three levels of initiation, transition, and breakthrough. A participant can exhibit more than one characteristic within each level of engagement, so the individual indicators may not equal the total number of participants. For example, with a sample of $N=90,84$ participants exhibited the "doing the activity" initiation behavior and 61 participants exhibited the "observing others at the exhibit" initiation behavior. If a participant exhibits just one of these behaviors, then that participant is considered to have reached the initiation stage. The same is true for the transition and breakthrough categories. Within each engagement 
level exists sub-categories of behavior that represent each level of engagement. Table 4 below demonstrates the frequency with which each individual behavior level was reached.

Table 4

Frequency of Individual Engagement Behavior Levels for All Participants

\begin{tabular}{lcc} 
Behavior Category & Frequency & Percent \\
\hline Initiation & & \\
$\quad$ Doing Activity & 84 & 93.3 \\
$\quad$ Observing Others at Exhibit & 61 & 67.8 \\
Transition & & \\
$\quad$ Repeating the Activity & 40 & 44.4 \\
$\quad$ Positive Emotional Response & 52 & 57.8 \\
Breakthrough & & \\
$\quad$ Referring to Past Experience & 22 & 24.4 \\
Seek/Share Information & 15 & 16.7 \\
Involved/Engaged & 33 & 36.7 \\
\hline
\end{tabular}

\section{Individual Engagement Levels}

Qualitative data showed that observations from the makerspace aligned well with the context-specific framework developed for science centers (see Chapter 3, Table 2). In the paragraphs below, the initial Visitor Based Learning Framework (VBLF) description is in italics and the specific observation example follows.

Initiation. The first engagement level used in the framework is the level of Initiation, which was reached by $100 \%$ of visitors and which consists of two components. The first behavior is Doing the Activity (observed in 93\% of participants), which is marked by the following activities: in passing, not done completely; doing the activity somewhat completely; doing the activity completely without further exploration or testing 
variables. Visitor 54, who reached only the initiation level, provides an excellent example of this behavior. Upon entering the makerspace, she waits briefly at the first station for a facilitator to come and explain instructions. When nobody shows up, she moves to the last station and begins playing with the dough. After a few minutes, she runs off to another part of the museum (McCubbins, field notes, July 10, 2015).

The second behavior is Observing Others at the Exhibit (observed in 68\% of participants), which is marked by the following activities: looking at the exhibit working, or someone doing the activity; watching the exhibit or person using the exhibit with expressed interest in the activity; interested in learning outcome or in learning the activity — visitor does the activity after observing. This behavior could be seen in Visitor 20, who only reached the initiation level. He noticed some people in the exhibit and so came to the station to see what was happening. He observed the hydraulics activities, watching some visitors. He left and then returned to the exhibit after a few minutes. $\mathrm{He}$ then joined a group and followed them around as they did the activities, observing from the table (McCubbins, field notes, June 29, 2015).

Transition. The second level of engagement is Transition, which was reached by $66 \%$ of visitors and which consists of two components. The first component is Repeating the Activity (observed in $44 \%$ of participants), which includes the following types of activities: doing the activity two or three times to attain a desired outcome or master the exhibit's function; enjoyment of the outcome; changing the variables to look for a different outcome. Visitor 53, who ultimately reached breakthrough levels, best exhibits these behaviors when participating in the squishy circuit activity. After learning about the difference between conductors and insulators, she begins testing different materials. She 
sorts the materials into two piles. When she gets a conductor she says "yay" and places it into the conductor pile. When she gets an insulator she says "aww" or "bummer" and places it into the insulator pile. She continues this process until she has tested all of the materials (McCubbins, field notes, July 9, 2015).

The second component is Expressing positive emotional response (observed in $58 \%$ of participants), which includes the following activities: smiling, pleased with exhibit; stronger signs of enjoyment such as laughter, verbal reference to enjoyment; obvious signs of eagerness to participate, excited disposition. Visitor 36, who reached breakthrough levels, demonstrates this disposition in his comments during the squishy circuit makerspace activity. While testing different materials to determine whether or not they are conductive, he selected the bell and connected it to the circuit. The light came on and his face filled with an expression of awe. "It worked!" he exclaimed (McCubbins, field notes, July 7, 2015).

Breakthrough. The third level of engagement is Breakthrough, which was reached by $51 \%$ of visitors and which is represented by three components. The first component is Referring to past experiences (observed in $24 \%$ of participants), which includes the following activities: reference to past experience with exhibit or science center; simple reference to comparable experience in visitor's life; reference to comparable experience in their life as well as making comparisons and deductions based on observations of similarities and differences. When entering the makerspace with her sister, Visitor 86, who reached breakthrough levels, recalled past information relating to the stop-motion animation activity. Her interaction with the facilitator is described below:

Facilitator: We are making movies today by taking a bunch of pictures and then putting them together really fast to make a video. 
Girl: I do that!

Facilitator: You do?

Girl: Yes.

Facilitator: On the computer?

Girl: On paper.

Facilitator: Oh, like a flipbook?

Girl: Yes!

(McCubbins, field notes, July 24, 2015)

The second component is Seeking and Sharing Information (observed in 17\% of participants), which can be seen in the following types of activities: calling someone over to look at exhibit, or ask them to explain an exhibit; reading signage, having conversations about exhibit and related science with staff or family member/friend; sharing experiences with others by explaining the exhibit to them, giving details about gained information and observations. During the squishy circuit activity in the makerspace, Visitor 29 demonstrated this level of breakthrough. After working for a while on her own in the space, she waved her mom over and went back to the first station. She started telling her mom what was happening with the materials and what she learned about insulators and conductors. She then took them with her to the next station and started explaining the conducting and insulating dough (McCubbins, field notes, July 6, 2015).

Finally, the third component is Engaged and Involved (observed in $37 \%$ of participants), which is represented by the following types of activities: engaging in inquisitive behavior; concentration and motivation are obvious, length of interaction is significant; experimenting, testing different variables, looking for different outcomes. This level of engagement is best represented by Visitor 13, who was engaged in the hydraulics activity in the makerspace. First, he began testing different variables with the syringe example by changing the amount of water in each syringe and changing the sizes 
of the syringes. He also decided he wanted to see how many little syringes of water it would take to fill the big one. He made a prediction and then began testing his hypothesis. After continuing to explore the exhibit for an extended period of time, his grandmother said to the facilitator, "Obviously this has captured his attention," (McCubbins, field notes, June 25, 2015). All of these examples provide insight into engagement behaviors for the makerspace overall, but we can also examine engagement levels for specific activities.

\section{Engagement Level by Activity}

Further analysis by activity can also provide information regarding engagement level. Five different activities were observed over the course of the research collection period; they were: Pollinators $(N=9)$, Hydraulics $(N=15)$, Open Lab $(N=5)$, Squishy Circuit $(N=37)$, and Stop Motion Animation $(N=24)$. A detailed description of these activities can be found in Appendix D. Using a Visitor Engagement Profile (VEP), we can examine what engagement looked like for each of these activities.

Hydraulics. Figure 2 represents the VEP for the Hydraulics Makerspace activity. In this activity, only $26.6 \%$ of visitors reached the breakthrough level of engagement. 


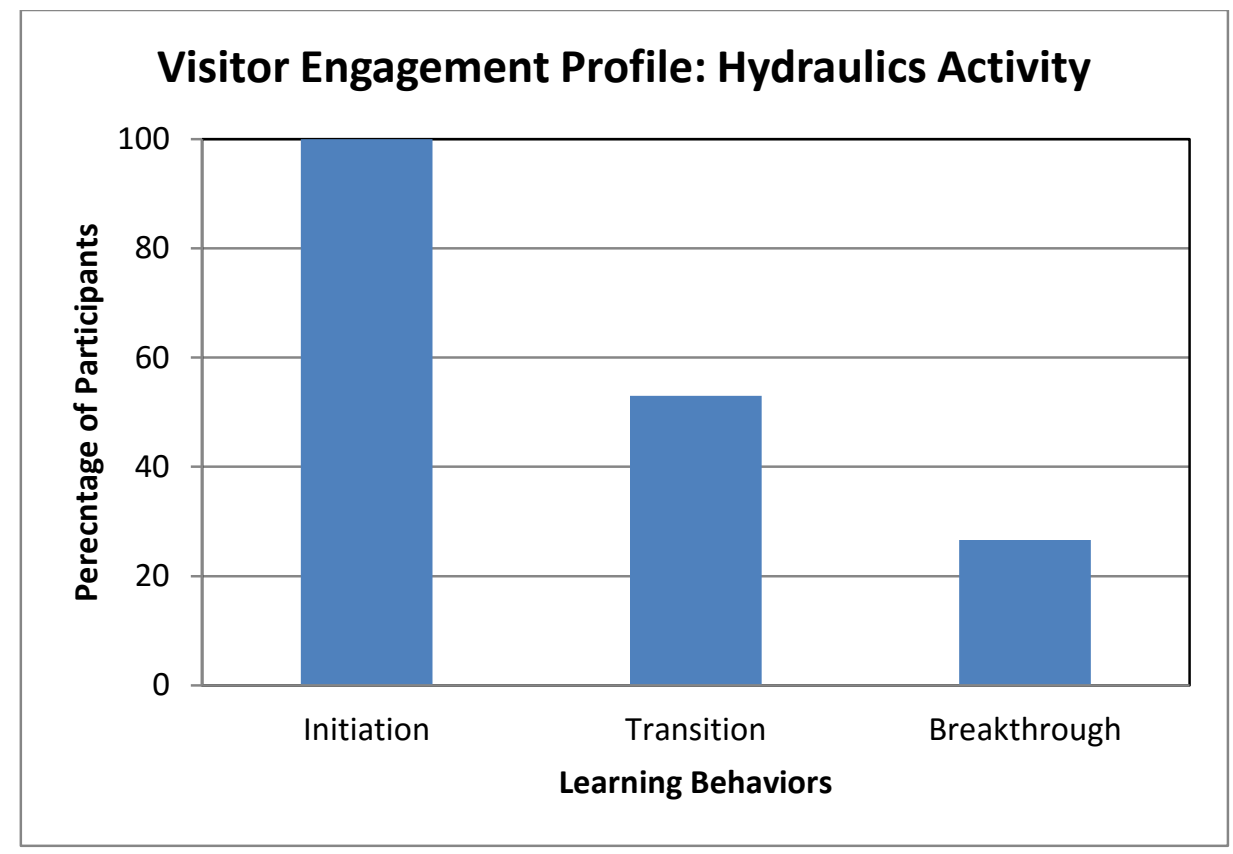

Figure 2. Overall VEP for hydraulics makerspace activity

The frequency can also be examined within each behavior level reached for the hydraulics activity. These results can be seen below in Table 5 .

Table 5

Frequency of Individual Engagement Behavior Levels for Hydraulics

Activity

Behavior Category Frequency Percent

Initiation

Doing Activity 13

86.6

Observing Others at Exhibit

8

53.3

Transition

Repeating the Activity

4

26.6

Positive Emotional Response

7

46.7

Breakthrough

Referring to Past Experience

Seek/Share Information

3

6.7

Involved/Engaged

1

26.6 
When examining the qualitative data for the hydraulics makerspace activity, some interesting trends emerge. Overall, visitors who stayed at the exhibit had a fairly easy time shifting from initiation to transition because they were engaged in the syringe activity, which involved the use of hydraulics to move one syringe with water when the other was pushed. However, disengagement quickly occurred for the next activity, which focused on creating origami and then incorporating hydraulics in order to make components of the origami move. This was very confusing for both the visitors and the facilitators and often resulted in a lack of breakthrough behaviors. Visitor 14, for example, went to the origami station and starting making something there, and she also saw syringes and balloons and so attached those and played with them, but she never was able to connect the concepts together to fully complete the activity (McCubbins, field notes, June 25, 2015). As a result, she only reached the transition level of engagement.

The facilitators played a major role in the lack of breakthrough as well because of how they approached the exhibit. This will be described in more detail below, but Visitor 22 is an excellent example of this observation. Not only did the facilitator build the entire origami creation for Visitor 22, but when finished, she gave him markers and told him to color it rather than demonstrate how to incorporate hydraulics into the creation, which was the initial focus of the activity. As a result, the visitor got bored and left the exhibit without completing the activity, only reaching the initiation level of engagement (McCubbins, field notes, July 1, 2015).

Squishy circuit. The VEP for the Squishy Circuit Makerspace activity can also be examined. Figure 3 shows that $51.1 \%$ of visitors experienced breakthrough during the Squishy Circuit activity. 


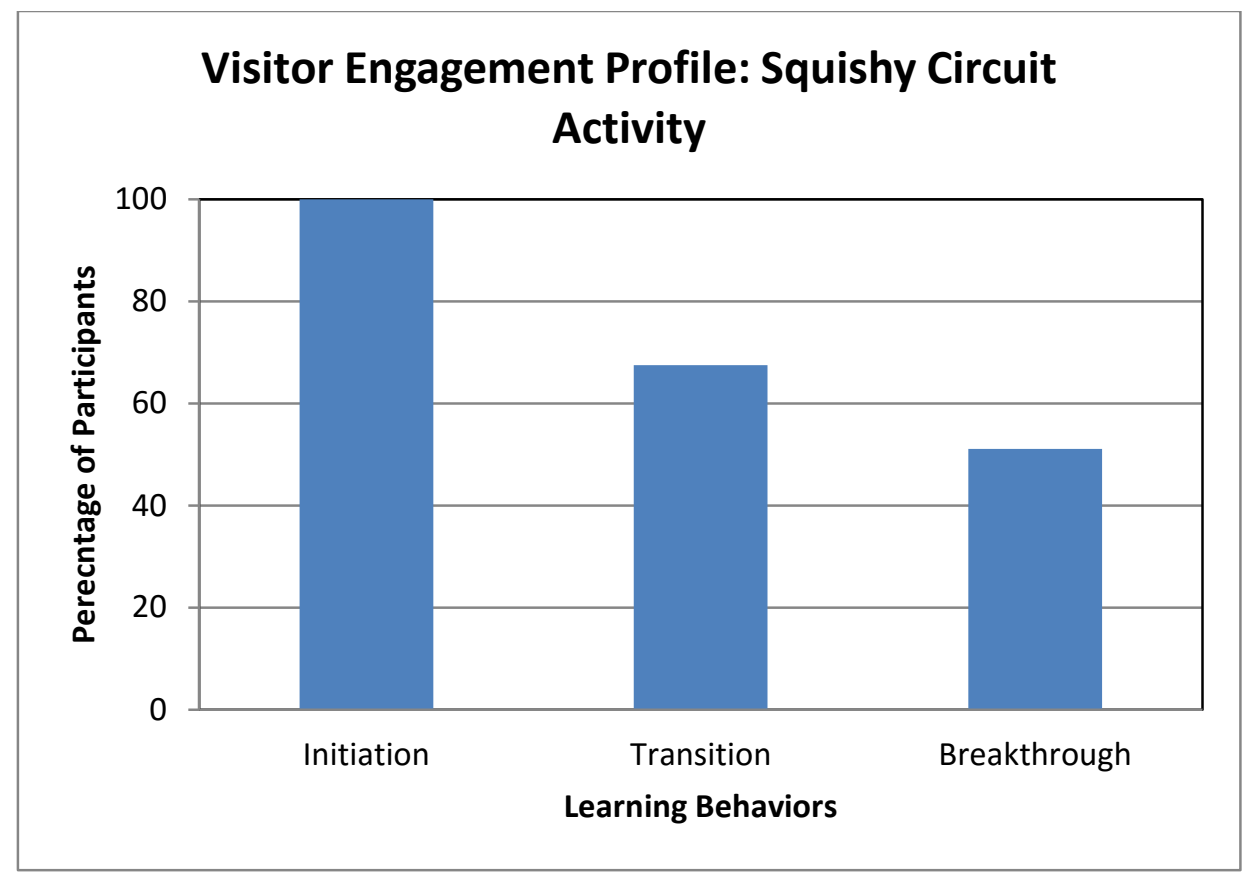

Figure 3. Overall VEP for squishy circuit makerspace activity

The frequency within each behavior level reached for the squishy circuit activity. These results can be seen below in Table 6 .

Table 6

Frequency of Individual Engagement Behavior Levels for Squishy Circuit Activity

\begin{tabular}{lcr} 
Behavior Category & Frequency & Percent \\
\hline Initiation & 36 & 97.3 \\
$\quad$ Doing Activity & 23 & 62.2 \\
$\quad$ Observing Others at Exhibit & & \\
Transition & 22 & 59.4 \\
$\quad$ Repeating the Activity & 19 & 51.4 \\
$\quad$ Positive Emotional Response & & \\
Breakthrough & 11 & 29.7 \\
$\quad$ Referring to Past Experience & 7 & 18.9 \\
Seek/Share Information & 11 & 29.7 \\
$\quad$ Involved/Engaged & & \\
\hline
\end{tabular}


Visitors to the Squishy Circuit activity experienced high levels of engagement based on the qualitative data, which accounts for the high breakthrough level for this exhibit activity. In particular, the activity which focused on using conducting and insulating dough was highly engaging because visitors could manipulate the dough and use knowledge from the previous activity about conductors and insulators to try and create a circuit. For example, visitor 26, who ultimately reached transition levels, began simply by observing others in the space. Once they moved to the dough station, she became involved, rolling her dough into a worm. The goal was to use the dough as a bridge to connect an LED to a battery and get it to light up. Her first worm did not work, so she decided to make a fatter worm. This time it worked, and she was pleased. She left the station but returned 20 minutes later to create more with the dough (McCubbins, field notes, July 6, 2015).

One issue that the quantitative data could not address but was revealed upon analysis of the qualitative data was that students seemed to develop some misconceptions about electricity and specifically conductors and insulators that went unaddressed during this makerspace activity. In the first activity of the squishy circuit exhibit, visitors are given a variety of materials and sort them into piles based on whether they are an insulator or a conductor. Often times, facilitators would encourage positive responses such as "yay" or "yes" when the LED worked (conductor) and negative responses such as "aww" or "bummer" when the LED did not work (insulator). Visitor 61 is a good example for how this language created a misconception with the visitor. Not only did she begin to use this language when testing her materials, but she came away with the impression that insulators are bad and conductors are good. This misconception, though 
verbalized during the activity, was never corrected or addressed by the facilitator (McCubbins, field notes, July 10, 2015). This visitor only reached transition level behaviors.

Stop motion animation. Finally, the VEP for the Stop Motion Animation activity can be analyzed in Figure 4 below.

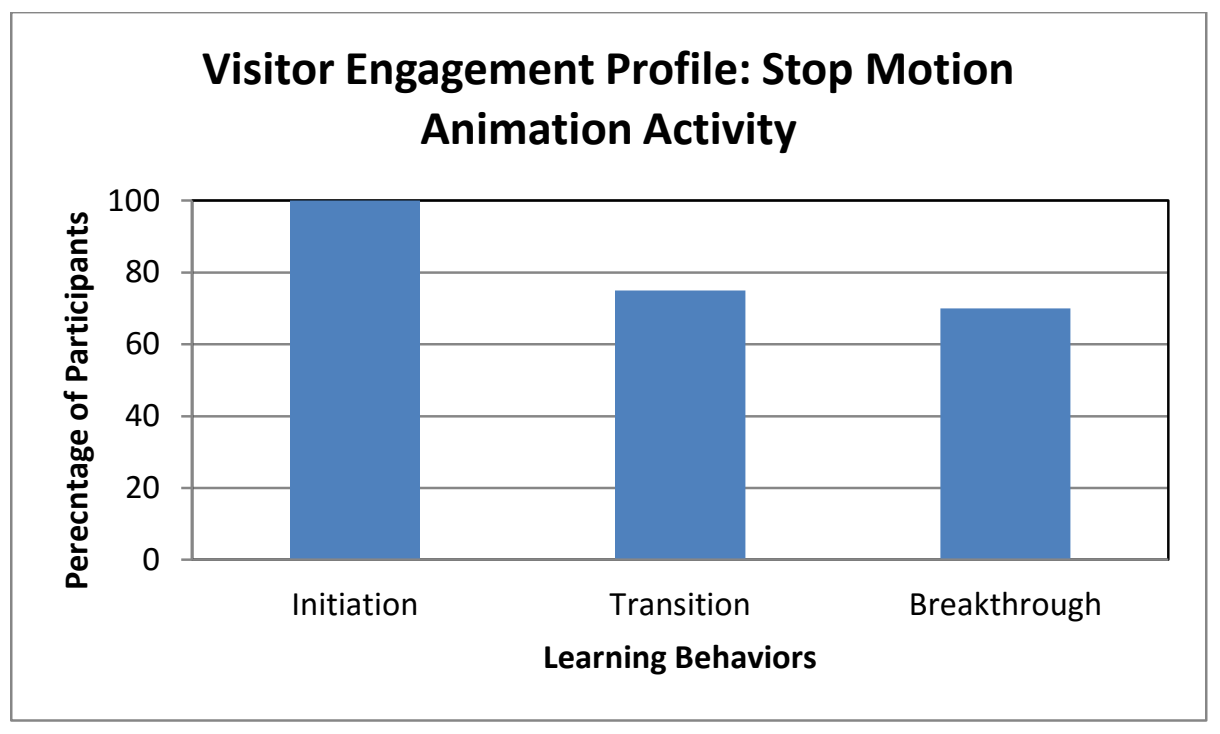

Figure 4. Overall VEP for stop motion animation makerspace activity

This activity has a unique VEP in that more than $70 \%$ of visitors reached breakthrough behavior. One factor in this higher level of breakthrough was the emphasis on teamwork and planning in this activity, which often led visitors to pair up with each other and encouraged many of the breakthrough behaviors such as seeking/sharing information and referring to past experiences. Visitor 80, for instance, came into the exhibit on her own, but once she saw two other visitors also making characters, she decided that she wanted to make her movie with them. They joined together as a group and began discussing what background they wanted to use and what they wanted their 
story to be about (McCubbins, field notes, July 22, 2015). This visitor reached

breakthrough levels of engagement.

The frequency within each behavior level reached for the stop motion activity can be seen below in Table 7 .

Table 7

Frequency of Individual Engagement Behavior Levels for Stop-Motion Animation

\begin{tabular}{lrr} 
Behavior Category & Frequency & Percent \\
\hline Initiation & & \\
$\quad$ Doing Activity & 23 & 95.8 \\
$\quad$ Observing Others at Exhibit & 23 & 95.8 \\
Transition & 9 & \\
$\quad$ Repeating the Activity & 18 & 37.5 \\
$\quad$ Positive Emotional Response & & 75.0 \\
Breakthrough & 4 & 16.7 \\
$\quad$ Referring to Past Experience & 5 & 20.8 \\
Seek/Share Information & 15 & 62.5 \\
$\quad$ Involved/Engaged & & \\
\hline
\end{tabular}

Also related to the high breakthrough levels for this exhibit was the fact that the focus on making a video often led to a lot of role-playing and acting-style conversations, especially from the young child category, and this ultimately resulted in the visitors staying actively engaged in the exhibit. For example, Visitor 73, who reached breakthrough levels, while waiting for his group to finish creating their characters, began playing with his character, making action noises and engaging the boy next to him (McCubbins, field notes, July 21, 2015). In another example, Visitor 87, who also reached breakthrough levels, decided that she and her group were going to use the premade Harry Potter characters for their stop-motion animation movie. They picked up 
some of the characters and started talking in funny voices, playing out a skit with the characters. Later, once they finished the story, they exclaimed, "That is the end of our movie!" and took a bow. Then they clapped for each other and laughed. (McCubbins, field notes, July 24, 2015).

\section{Influences on Engagement}

Independent from a specific activity occurring in the makerspace but also influencing the engagement occurring within the exhibit were the roles that the parents and facilitators played throughout the learning experience, as well as the pedagogical approaches to the activities and the ways in which the space was laid out. These will each be described in more detail in the sections that follow.

Influence of parental dialogue. The parent was critical in encouraging persistence and breakthrough behavior based simply on whether they were communicating in a positive or negative way with their child. Positive dialogue often resulted in lots of questioning and critical thinking from the visitor, resulting in more engagement and an excited disposition. Visitor 90, for example, was participating in the stop-motion animation exhibit. After spending a half hour creating his characters and designing his movie timeline, his dad says 'I'm getting excited to see this video you're making." These words of encouragement results in a smile from the boy and an increased focus on finishing the pictures and creating his movie (McCubbins, field notes, July 24, 2015). As a result, he reached breakthrough levels of engagement. Also participating in the stop-motion animation exhibit and also reaching breakthrough levels of engagement was Visitor 71, who received praise from her mother. "When you're done being a genius, we have lunch over at the table." The girl grinned and continued working on her project. 
There were computer glitches, so she left to eat lunch while the volunteer worked on it and then returned to finish her movie (McCubbins, field notes, July 20, 2015).

Negative dialogue also had an influence on learning behavior, often resulting in a loss of interest and disengagement or moving on to the next exhibit. For example, Visitor 5, who only reached initiation levels, was creating a creature as part of the pollinator activity. He grabbed materials and glued them together. Then he looked for something to represent the eyes. "Maybe we can use this?" he said, asking his mom. "That would be ugly," she says. The mom grabs some fabric, glues it on the creature, and then has the boy put the supplies away and they leave (McCubbins, field notes, June 18, 2015). Visitor 1 also experienced negative dialogue from his mom during the pollinator activity, though he reached the transition level of engagement. He wanted to use some tools that were available in the space, but his mom said "They don't want you to play with something sharp, put it back." Even after the facilitator told her it was okay for him to use the tools, she said no. He looked around a little bit, did some of the activity, and then left the exhibit (McCubbins, field notes, June 18, 2015).

Time constraints also influenced learning with regard to parent dialogue. Many visitors who were engaged and approaching breakthrough level ended up not reaching breakthrough because their parent told them they had to leave or move on to another exhibit. Visitor 36, for example, was completing the squishy circuit conductor and insulator activity and was ready to move to the dough station. "Mom, I want to try this" he said. "We have 5 minutes," replied the mom. "We have to go upstairs." The boy then leaves and goes upstairs (McCubbins, field notes, July 7, 2015). Although this cut into his time in the exhibit, he still managed to reach the breakthrough level of engagement. 
Influence of facilitator. Facilitators also had the ability to encourage or discourage breakthrough with their language as well as their knowledge and approach to the activity. During times when the facilitator used positive language by questioning or using scientific language, visitors were more likely to recall previous knowledge and experiment with the activities. For example, during the squishy circuit activity the facilitator greets Visitor 48 by saying "do you want to learn about electricity today?" to which the visitor responds "Sure." Then the facilitator asks, "What do you already know about electricity?" From this point on, the visitor recalls prior knowledge and is engaged in trying to learn more to add to her existing knowledge about electricity and reaches breakthrough engagement levels (McCubbins, field notes, July 9, 2015). In another example, Visitor 86, who also reached breakthrough levels of engagement, is working on her stop-motion animation video. They have already created the pictures and loaded them into the software and are watching a preview of the video. The girl says she wants it to go a little bit slower, so the facilitator asks, "If the current frames per second are at 12, what should we change it to in order to make it go slower?" The girl thinks for a moment and then says, "Let's try 10!" They try it and it works, and the girl is pleased" (McCubbins, field notes, July 24, 2015).

Also, the use of praise and encouragement from the facilitator was important in order to engage the visitor so that they could reach breakthrough levels of engagement. Visitor 82 is a perfect example of how praise and encouragement from both the parent and the facilitator can lead to breakthrough. Below is an excerpt of the field notes for Visitor 82, after she has entered the makerspace during the stop-motion animation activity and completed assembly of her character: 
Facilitator: Do you want to make a movie now?

Girl: I don't know.

Mom: Why don't you try?

The girl makes some final touches on her character and then follows the facilitator into the space with the movie backgrounds.

Facilitator: Now, if you want, we can pick a scene for your movie, and you can pick any other characters you want to be in your movie with you. She picks up the Dumbledore and decides on her scene. The facilitator explains that it is really important to move the characters slowly for the pictures so it makes it look like they are moving. Then she asks,

Facilitator: Do you want them to have a dance party?

Girl: "Yes!"

....(they work on the individual shots for the movie)....

When they get toward the end, the facilitator asks,

Facilitator: What do you want to happen at the end?

Girl: They should all fall over!

Facilitator: that's great!

They finish the pictures, then they go create the movie on the computer. Another girl comes over to join them and see what the movie is about. They laugh and watch the movie three more times before leaving.

(McCubbins, field notes, July 22, 2015)

This excerpt demonstrates how the parent and facilitator helped the visitor go from being nearly finished with her engagement (following the creation of her character) to reengaging her in the activity to the point where she reached breakthrough levels of behavior.

Just as the positive dialogue and questioning can have a positive effect on engagement, negative dialogue, lack of interest, and lack of knowledge or attention from the facilitator can all have a negative influence on engagement. First, the qualitative data showed a few instances in which breakthrough was prevented because a facilitator discouraged visitors from trying something, usually because of time constraints of either the visitor or the makerspace. For example, Visitors 65 and 66 came in together and decided they wanted to build a birdhouse. After considering what materials they wanted to use, they began to assemble the wood for their birdhouse. The facilitator approached 
them and asked how long they planned to spend in the makerspace because building with wood would take a long time. The girls decided they should look for different materials. They couldn't find anything they liked so they abandoned their ideas for the birdhouse and left the space without doing any activities. As a result, these visitors only reached the initiation level of engagement (McCubbins, field notes, July 11, 2015).

Lack of interest from the facilitator also had a negative influence on engagement. This was especially apparent for the hydraulics activities. After the first two days of the activity, the facilitators seemed tired of the topic. They were no longer explaining the connection to hydraulics in each of the activity stations and instead seemed to be focused simply on getting visitors to the origami station where they encouraged them to sit there and create and color origami, which had nothing to do with the original hydraulics activity of creating moveable parts on the origami. This happened repeatedly and definitely influenced the ability for visitors to experience breakthrough behavior (McCubbins, field notes, June 29, 2015).

Finally, lack of knowledge and attention led to disengagement of visitors which prevented them from reaching breakthrough. This was especially a problem when there was one facilitator and a handful of volunteers running the makerspace. In many cases, the facilitator had the knowledge about the stations but did not communicate that to the volunteers very well. As a result, when exhibits were crowded or the facilitator was busy, visitors who were working with the volunteers were often left without core information needed to help encourage the breakthrough behaviors. The best example of the influence of facilitator lack of knowledge and attention is seen in the field notes for Visitors 73 and 74. The brother and sister came into the makerspace together to create their own stop- 
motion animation video. Initially they were excited, adding designs to their characters and choosing their backgrounds. A facilitator introduced the activity to them and got them started, and then a volunteer followed them around as they selected their scene for their movie. The volunteer does not know how to set up the camera to take the pictures or how to assemble the movie, so they all wait for the facilitator to finish helping another person. The sister decides that she no longer wants to make a movie and leaves the space. The brother watches another group who has finished their movie and then leaves the space as well. At this point the facilitator is still helping another person and the volunteer still does not know how to run the activities. The end result was that both visitors only reach the transition level of engagement (McCubbins, field notes, July 21, 2015). The ability for the facilitator and volunteers to have both the content knowledge to guide the visitors through engagement as well as the time to help visitors when they get stuck is crucial for breakthrough behavior.

Influence of pedagogical approach. Perhaps the most important factor in determining whether a visitor will reach breakthrough levels of engagement or not is the pedagogical approach taken by the facilitator for the activity. This was most obvious in the squishy circuit makerspace activity. In the following excerpt from Visitor 30, who reached the breakthrough engagement level, the facilitator uses a more constructivist approach in teaching visitors about conductors and insulators as well as the connection to the dough being used in station two:

Facilitator: Today we are learning about electricity. Do you know anything about electricity?

Boy: Well I know it powers my video games.

Facilitator: Exactly! So, we have a wire and a battery and a lightbulb and when we connect them all together, it makes the lightbulb light up. Why do you think that is? 
Boy: Because we connected the circuit.

Facilitator: Good, now I want you to take any of these materials and see if you can connect them to try and make a bridge to get the lightbulb to light up. (the boy starts grabbing some of the materials). Do you want to try the bell?

Boy: Yes

Facilitator: Okay touch the wire anywhere on the bell. Does the lightbulb light up?

Boy: Yes

Facilitator: We have a special word for that. We call that a conductor.

Now try something that you think will not make it light up. (the boy grabs a piece of plastic and tests it).

Boy: This doesn't work.

Facilitator: We have a special word for that too. It's called an insulator. .....(the boy continues testing the remaining materials, discussing with his mom as he completes the activity. Then they move over to the dough station)....

Facilitator: We have two special kinds of dough. One is conductive and one is insulative. Can you make a circuit out of the dough?

The boy starts building with the dough. He connects two parts of the dough and makes the lightbulb light up. When he pushes the dough together, the light goes out, and when he separates them, the light comes on. The facilitator then directs him to the third station where he creates his own creature with eyes that light up. He finishes his creation and shows his mom. She takes information about how to make the dough at home and they leave the space.

(McCubbins, field notes, July 7, 2015)

This approach allows the visitor to be more engaged and maintain interest in the topic in order to reach breakthrough behavior. In the case of Visitor 46, however, the more didactic approach from the facilitator led to boredom and frustration, ultimately resulting in the visitor leaving the space. Though he is engaged, he does not reach full breakthrough levels because of this boredom and frustration.

Facilitator: We are doing some experiments with electricity today. What do you know about electricity?

Boy: I know it is electric.

He says he doesn't know more information, though he is able to connect the light to the battery, so at least intuitively he knows about circuit in some sense. The staffer explains to him what conductors and insulators are and then tells the boy to sort the materials into two piles. When he finishes, he moves over to station two with the dough. He is watching 
while the facilitator shows him the difference between the two doughs. Once they show him, he moves to the third station and starts building a creature. He is only using conductive dough, which means he did not remember or understand what the facilitator told him about the doughs in the second station. He does not know what to do next, or how to get things to light up. He seems confused about what is supposed to happen at the station. He decides to go play somewhere else and leaves.

(McCubbins, field notes, July 9, 2015)

Note that the pedagogies vary greatly between facilitators. In the first case, the facilitator had the visitor test one or two materials, observe the difference, and explain what was happening. Then the facilitator gave the visitor the terminology for what they were describing and had them sort the remaining materials into two piles based on these new definitions. Once the visitor moved to the second station, the facilitator challenged them to try and make a circuit using the dough, which led the visitors to experiment with the dough to figure out what would work. In the second case, however, the facilitator told them the definitions immediately and then had the visitor sort the materials based on those definitions. In the second station, the facilitator simply lectured that one dough would make the LED light up whereas the other dough would not. The facilitator did not use the terminology from the first activity and simply demonstrated for the visitor rather than letting the visitor try to figure out the difference between the doughs. This approach ultimately led the visitor to frustration and resulted in him leaving the space.

Influence of exhibit layout. Finally, the way in which the space was set up influenced engagement levels. In some instances, there were too many activities at once or resources were visible that were not available to use, which often led to disengagement of the visitor after she found out she could not use the materials she wanted. Visitor 78, for example, was working on her character for the stop-motion animation activity. Another staff member from the museum came in and told the facilitator that she needed 
some things made for another event that was happening later in the week. It required the volunteers to use a hammer, which made a lot of noise and was difficult to work alongside with. Not only did Visitor 78 leave the space, only having reached the transition level of engagement, but the noise attracted people to the space who thought they would get to make things with the hammer. The staff had to keep explaining that the activity was not the hammer but rather creating characters for stop-motion animation. A lot of visitors, especially male visitors, did not want to create "dolls" so they left the space (McCubbins, field notes, July 21, 2015). The facility itself was also problematic for some of the audience because of the height of the tables. Visitor 59 was very interested in the squishy circuit activity, but he was in a wheelchair and the height of the table was at his nose. This made it difficult for him to see or participate in any of the activities. He and his mom decided to leave and go to the next floor where they could paint and as a result, only reached the initiation level of engagement (McCubbins, field notes, July 10, 2015). As these examples demonstrate, even the layout and components of the space can influence the engagement behaviors that occur in the exhibit.

\section{Research Question Two}

The second research question was focused on answering what relationships exist between engagement level and age and gender. To answer this question, data from the observation protocol were used to run correlation and statistical significance tests with cross-tabs analysis. The field notes were used to contextualize the quantitative data. To address Research Question 2, the demographics were analyzed in relation to the engagement levels. In terms of demographics, $34.4 \%$ were classified as "young child," meaning that they were between the ages of zero and six. Another $62.2 \%$ were classified 
as "child," which represented ages six through ten. Finally, 3.3\% were classified as "preteen," representing ages 11 through $13(N=90)$. As explained in the methodology chapter, participant ages were determined by the exhibit facilitator who asked each visitor his age as he entered the exhibit. These frequencies can be seen in Figure 5 below. There was an almost equal split for gender, with males representing $51.1 \%$ of the participants $(N=90)$.

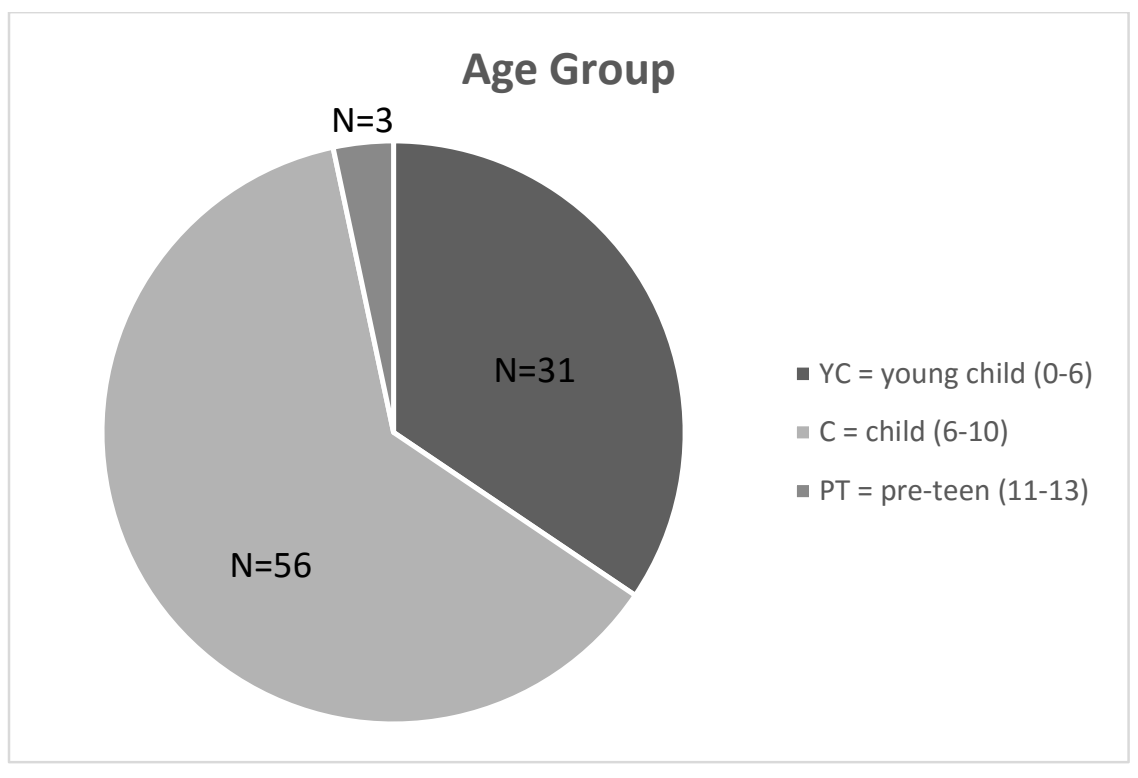

Figure 5. Percentage of participants within each age group

These frequencies allow us to conduct a further analysis of engagement level by looking more specifically at trends among gender and age groups.

\section{Engagement Level by Gender}

An analysis of the overall makerspace engagement level by age group and gender was conducted. Figure 6 represents the VEP according to gender. As can be seen below, males and females were just as likely to engage in transitional behaviors, and males were more likely to engage in breakthrough behaviors overall than their female counterparts. 


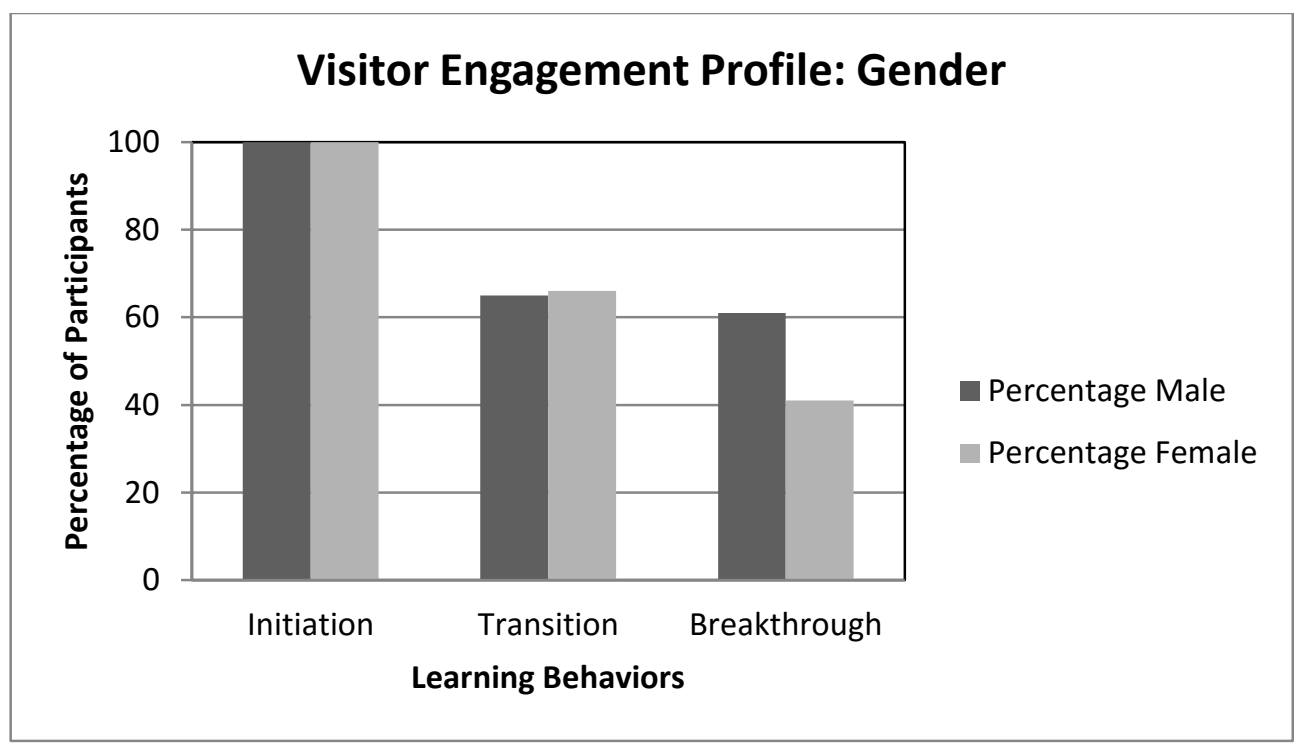

Figure 6. Overall VEP for learning behaviors based on gender

Although some differences were present, Table 8 below shows that the results were not statistically significant at the .05 level. Males and females were just as likely to exhibit transition behaviors and the difference was not statistically significant, Pearson $\chi^{2}(1$, $N=90)=0.005, p=0.945$, Cramér's $V=0.007$. Males were also more likely to engage in breakthrough behaviors overall than females, though again the results were not statistically significant at the 0.05 level, Pearson $\chi^{2}(1, N=90)=3.586, p=0.058$, Cramér's $V=0.200$.

Table 8

Chi-Squared Test Statistics and Significance for Learning Behaviors by Gender $(d f=1)$

\begin{tabular}{lllll}
\hline & $N$ & Pearson $\chi^{2}$ & Asymp. Sig. & Cramér's $V$ \\
\hline Overall Transition Behavior & 90 & 0.005 & 0.945 & 0.007 \\
$\begin{array}{l}\text { Overall Breakthrough Behavior } \\
\begin{array}{l}\text { Breakthrough Behavior- } \\
\text { Referring to Past Experiences }\end{array}\end{array}$ & 90 & 3.586 & 0.058 & 0.200 \\
\hline
\end{tabular}
$* \mathrm{p}<0.05$ 
While none of the overall VEPs were statistically significant at the 0.05 level, some significance did emerge when the learning behavior categories were broken down into their individual components. A two-way contingency table analysis (see Table 8) found that the specific breakthrough behavior of referring to past experiences was statistically more likely to occur for males than it was for females, showing significance at the 0.05 level, Pearson $\chi^{2}(1, N=90)=5.445, p=0.020$, Cramér's $V=0.246$. Of the 44 female participants, only six of them exhibited this breakthrough behavior, and fewer than half of the male participants exhibited that behavior. Figure 7 below shows the behavior of males and females in the "breakthrough-referring to past experiences" behavior category.

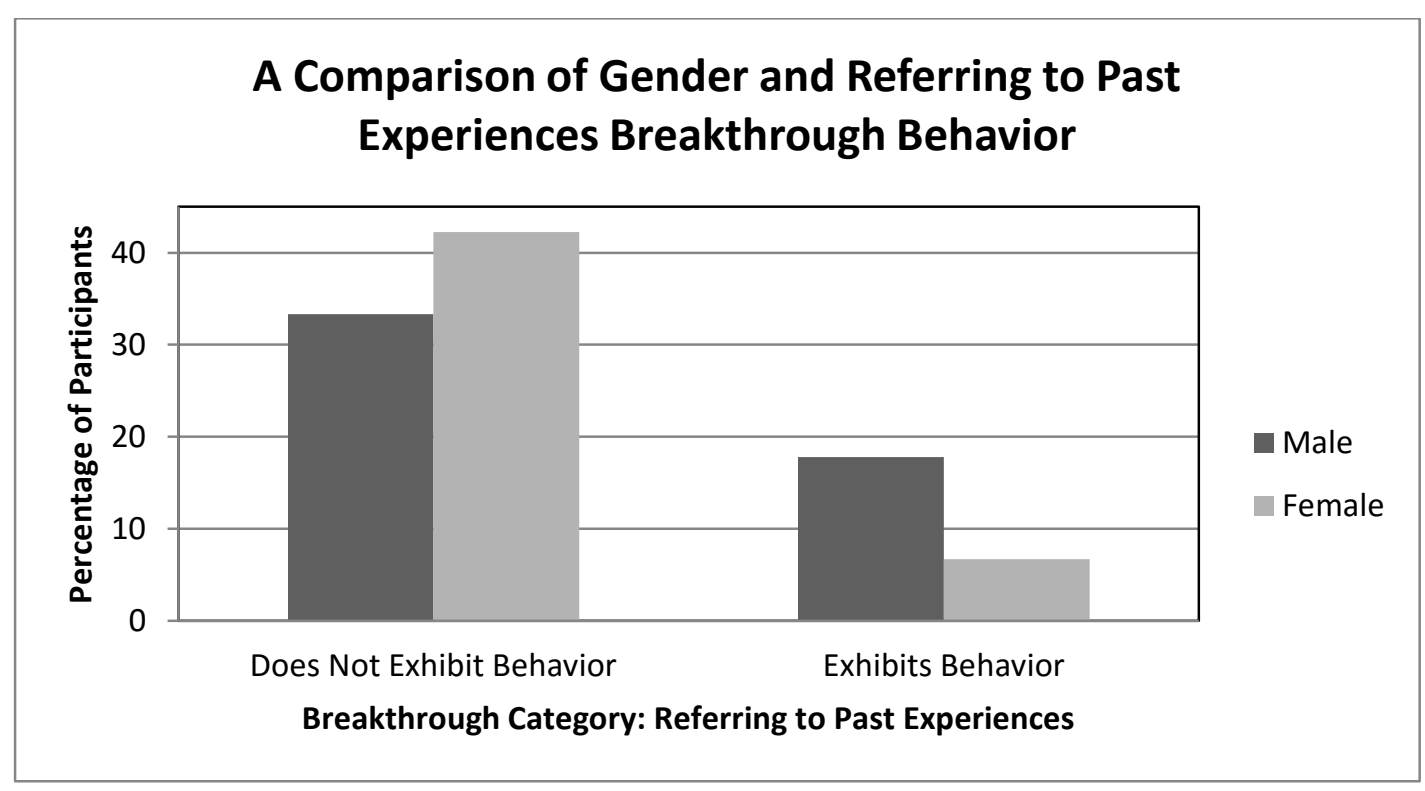

Figure 7. A comparison of gender and referring to past experiences breakthrough behavior

An analysis of the field notes also gives us insight into the differences between male and female participants in terms of how they approached the makerspace exhibit 
activities. Both genders showed excitement, but their excitement stemmed from different areas. Females were more likely to show excitement when they were able to share their creations with others, in particular their parents. For example, after Visitor 81 finished constructing a necklace for her stop-motion animation character, she excitedly took her creation out of the exhibit in order to go show her parents before returning to finish the activity (McCubbins, field notes, July 23, 2015). Males, on the other hand, were more likely to show their excitement after accurately predicting what would happen in the activity. For example, Visitor 25, who reached breakthrough levels, showed lots of excitement during the squishy circuit activity when trying to determine how to alter his creation. After building multiple creations already out of conductive and insulating dough, this time he adds some LED lights as eyes, and the following exchange occurs:

He starts putting the wire to the dough to see the LEDs light up. Boy: I wonder what would happen if I moved this here? He points to another section of the dough.

Facilitator: I don't know what will happen. Why don't you try it? He waits for a battery and then tries it and gets both lights to light up.

Facilitator: It works!

Boy: Yes!

They take a picture and then he pulls the wires up, folds up the dough, and leaves.

(McCubbins, field notes, June 25, 2015)

Males exhibited more of this hypothesis-testing behavior, and the use of their prior knowledge to help determine what would happen next was often factor in their excitement.

Another difference between males and females in their behavior related to how they dealt with frustration and difficult tasks. Although males experienced more breakthrough overall, they were more likely to exit the exhibit when faced with difficult 
or frustrating tasks. For example, Visitor 62 came to the makerspace during an open lab day and decided he wanted to make a robot out of wood. He first struggled with using the saw, and even after help from dad, he decided that wood was the wrong material. He then tried to create the robot out of cardboard and Styrofoam. This was also unsuccessful, so he decided to put his materials away. When the facilitator asked what he made, he tells her, "Nothing. I tried but it was really hard," and then he left the exhibit (McCubbins, field notes, July 10, 2015). Females, however, were more likely to be patient and persist when confronted with frustration or difficult tasks. For example, during the squishy circuit activity, Visitor 40 experienced some frustration with creating her creature out of dough because she could not get the eyes to light up. Below is an excerpt of the dialogue between the visitor and her mom:

They move over to the other table to make their creatures. The girl is having trouble getting the eyes to light up, even though the LEDs are placed in the correct (conductive) dough.

Mom: Does this work here?

Girl: No. It's not connected. They are still trying to get the eyes to light up.

Girl: We need more dough. The girl goes to get more conductive dough and adds it to her creature. The eyes light up.

Girl: It's working! They continue building for about 20 minutes, creating something and then rolling it into a ball and redoing it again. They finish and then leave to go explore the rest of the museum.

(McCubbins, field notes, July 7, 2015)

Although females were more likely to be patient and persistent, they were also more likely to disengage and leave the exhibit when given the option by the facilitators. Because the makerspace often had multiple activities happening at a time, all related to the same theme, the facilitators often used language that suggested visitors could continue to experiment at one station or move to the next. Based on the field notes, a 
majority of the male visitors responded to that question by proceeding to the next station, whereas female visitors such as Visitor 15 were more likely to choose to leave the exhibit and go play in another area of the museum, only reaching the initiation level of engagement (McCubbins, field notes, June 25, 2015).

Finally, male and female visitors were different in that the male visitors exhibited greater instances of distraction by other things happening within the makerspace or the museum as a whole, based on field notes collected during the study. Visitor 44, for example, entered the makerspace to do the squishy circuit activity. He stayed engaged in the activity until he moved to the last station, at which point he noticed the wood and tools blocked off in a separate part of the makerspace. When he asks if he can play with the wood and tools, he is informed that those are not available in today's activities. He decides to leave the makerspace and go to another exhibit (McCubbins, field notes, July 9, 2015). These kinds of distractions also led to the male visitors appearing bored or disengaged when they were observing rather than doing activities. For example, Visitor 69 was working with his sisters to create a stop-motion animation video. While constructing his character, he was engaged, but when they started to create the movie and only one person could take a picture or move the characters at a time, he became bored and decided to leave the makerspace (McCubbins, field notes, July 20, 2015).

\section{Engagement Level by Age Group}

Aside from the gender breakdown, we can also examine the VEP by examining the behavior breakdown based on age groups. In this analysis, the young child and child categories were comparable. In the transition category, those classified as "young child" (ages 0-6) were more likely to experience the transition learning behaviors than those 
classified as "child" (ages 6-10), and those classified as "child" were more likely to experience overall breakthrough learning behaviors than those classified as "young child." Figure 8 below represents the VEP according to age group.

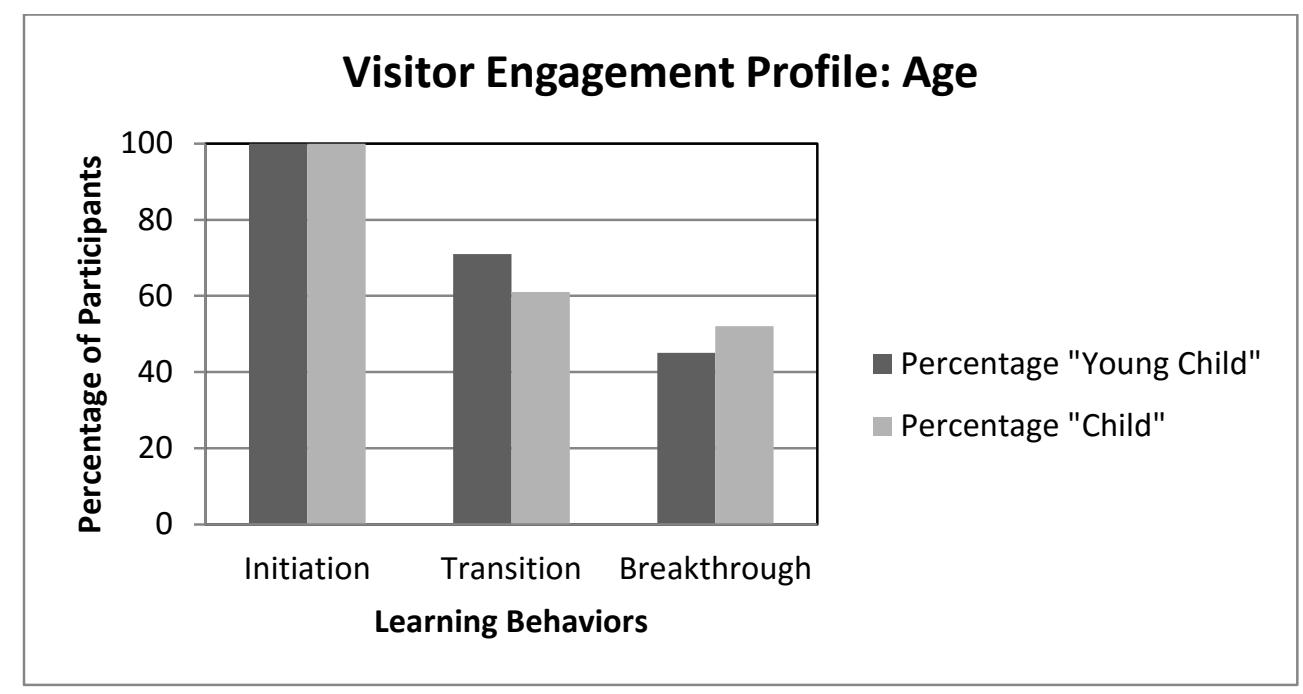

Figure 8. Overall VEP for learning behaviors based on age

Although the transition behavior category showed some difference between the "child" and "young child" age groups, Table 9 below shows that the results were not statistically significant, Pearson $\chi^{2}(2, N=90)=2.560, p=0.278$, Cramér's $V=0.169$. In the breakthrough category, "child” visitors were more likely to experience overall breakthrough learning behaviors than "young child" visitors, though the results were not statistically significant, Pearson $\chi^{2}(2, N=90)=3.319, p=0.190$, Cramér's $V=0.192$.

Table 9

Chi-Squared Test Statistics and Significance for Learning Behaviors by Age Group $(d f=2)$

\begin{tabular}{lllll}
\hline & $N$ & Pearson $\chi^{2}$ & Asymp. Sig. & Cramér's $V$ \\
\hline Overall Transition Behavior & 90 & 2.560 & 0.278 & 0.169 \\
Overall Breakthrough Behavior & 90 & 3.319 & 0.190 & 0.192 \\
\hline
\end{tabular}
$* \mathrm{p}<0.05$ 
Correlation coefficients were also computed between age group, gender, and the three learning behavior categories. The results of the correlational analyses are presented in Table 10 below and show that only one of the correlations was statistically significant at the 0.05 level. In general, the results suggest that the five categories are not related, with the exception of transition and breakthrough behaviors, though there was some correlation between gender and breakthrough behavior. In the case of the relationship between transition and breakthrough behaviors, the results suggest that those who reach the transition level are statistically more likely to reach the breakthrough level than those who do not. This makes logical sense given that the transition level is often required in order to move from the initiation behavior level to the breakthrough behavior level.

Table 10

Correlations Among Age, Gender, and Learning Behaviors $(N=90)$

\begin{tabular}{|c|c|c|c|c|c|}
\hline & Age Group & Gender & $\begin{array}{l}\text { Initiation } \\
\text { Behavior }\end{array}$ & $\begin{array}{c}\text { Transition } \\
\text { Behavior }\end{array}$ & $\begin{array}{c}\text { Breakthrough } \\
\text { Behavior }\end{array}$ \\
\hline Age group & 1 & -0.013 & $---^{a}$ & -0.028 & 0.139 \\
\hline Gender & -0.013 & 1 & $--^{a}$ & 0.007 & $-0.200 *$ \\
\hline $\begin{array}{l}\text { Initiation } \\
\text { Behavior }\end{array}$ & $--^{\mathrm{a}}$ & $---^{a}$ & $--^{a}$ & $--^{a}$ & $--^{\mathrm{a}}$ \\
\hline $\begin{array}{l}\text { Transition } \\
\text { Behavior }\end{array}$ & -0.028 & 0.007 & $--^{a}$ & 1 & $0.414 *$ \\
\hline $\begin{array}{l}\text { Breakthrough } \\
\text { Behavior }\end{array}$ & 0.139 & $-0.200 *$ & $--^{a}$ & $0.414 *$ & 1 \\
\hline
\end{tabular}

a. cannot be computed because at least one of the variables is constant.

When examining the qualitative data, some interesting distinctions emerge between the young child and child categories. First, young child visitors exhibited the "observing others" initiation behavior more than child visitors, who exhibited the "doing the activity" initiation behavior more frequently. In many cases, the young child visitor 
watched as a parent completed an activity or built a creation, with the young child providing input along the way. In this way, the parent role is focused on completing the activity for the child while they watch. For example, during the pollinator activity, Visitor 2, who reached the transition level, was creating a butterfly. She watched as her dad glued things together. Occasionally, he would ask her "where do you want to glue this piece?" and she would respond by pointing to her creation. Finally, the dad asks "anything else?" and the girl decides she is finished (McCubbins, field notes, June 18, 2015). Visitors who fit into the child category, on the other hand, tended to complete the task themselves. If parents were involved, their role was to observe or to help with one part of an activity while the child visitor was engaged in another part of the activity. Rarely was the child visitor not engaged in that way. During the stop-motion animation activity, for example, Visitor 78, who reached breakthrough levels of engagement, was working on creating a character for her movie. Below is the exchange that occurred:

The girl comes in with her mom to create a character. The mom is cutting out the pieces while the girl decides what part of her character she is going to color.

Girl: I'm making Rapunzel. She decides to take some yarn and braid it together to create the long hair.

Girl: Can you help me with her? Her dad goes with her to the hot glue station so they can glue on some pieces to the character. She finishes gluing and then continues to work on her character while her dad takes a phone call. He comes back and asks, Dad: What are you going to put on your character now? She tells him she is adding purple. She looks around the table of materials, pensive, trying to decide what she is going to add next.

(McCubbins, field notes, July 21, 2015) 
Another difference between young child visitors and child visitors was how they communicated while in the space. Field notes show that young child participants typically communicated with their parents or with the volunteers and facilitators within the space, whereas child participants tended to communicate with their peers. For example, Visitor 43, who reached breakthrough engagement levels, entered the makerspace to do the squishy circuit activity. He listened to an explanation of insulators and conductors and then began testing different objects to determine which category they fit into. When another visitor approached the station, he turned and started explaining what conductors were to the new person (McCubbins, field notes, July 9, 2015). Another difference in communication focused on the use of negative or fixed-mindset language among the child participants. Visitors made comments such as "this is going to be hard" (Visitor 40), or "I don't know if I can do that" (Visitor 82), or "I'm not good at that" (Visitor 19). In some cases, the facilitator was able to encourage them to continue the activity and in other cases the visitor left to go explore the rest of the museum. None of this fixed mindset language was observed at the young child level.

\section{Research Question Three}

The third research question was focused on answering how the makerspace engagement levels in this museum compare to engagement levels in traditional museum exhibits. Data from the observation protocol were used to create the overall Visitor Engagement Profile (VEP) and chi-square analysis was run. The VEP was then compared to what the literature has determined to be the expected engagement level based on level of interactivity and previous studies using the observation protocol instrument. The field notes were used to contextualize the quantitative data. Based on previous research from 
museums in Canada and the U.S. over the last decade, exhibits which are designed to involve visitors in hypothesis testing and meaning making should produce a VEP with at least $50 \%$ of visitors demonstrating breakthrough behaviors (Barriault, 2008). Although makerspaces have not been included in previous studies since they focus on traditional museum and science center settings, makerspace activities often center around hypothesis testing and meaning making; therefore, we would expect a VEP with 50\% breakthrough behavior or higher. In fact, that is exactly what the data shows. Figure 9 represents the overall VEP, demonstrating that initiation was the most frequent form of engagement, followed by transition and then breakthrough. Over $51 \%$ of participants reached the breakthrough stage of engagement.

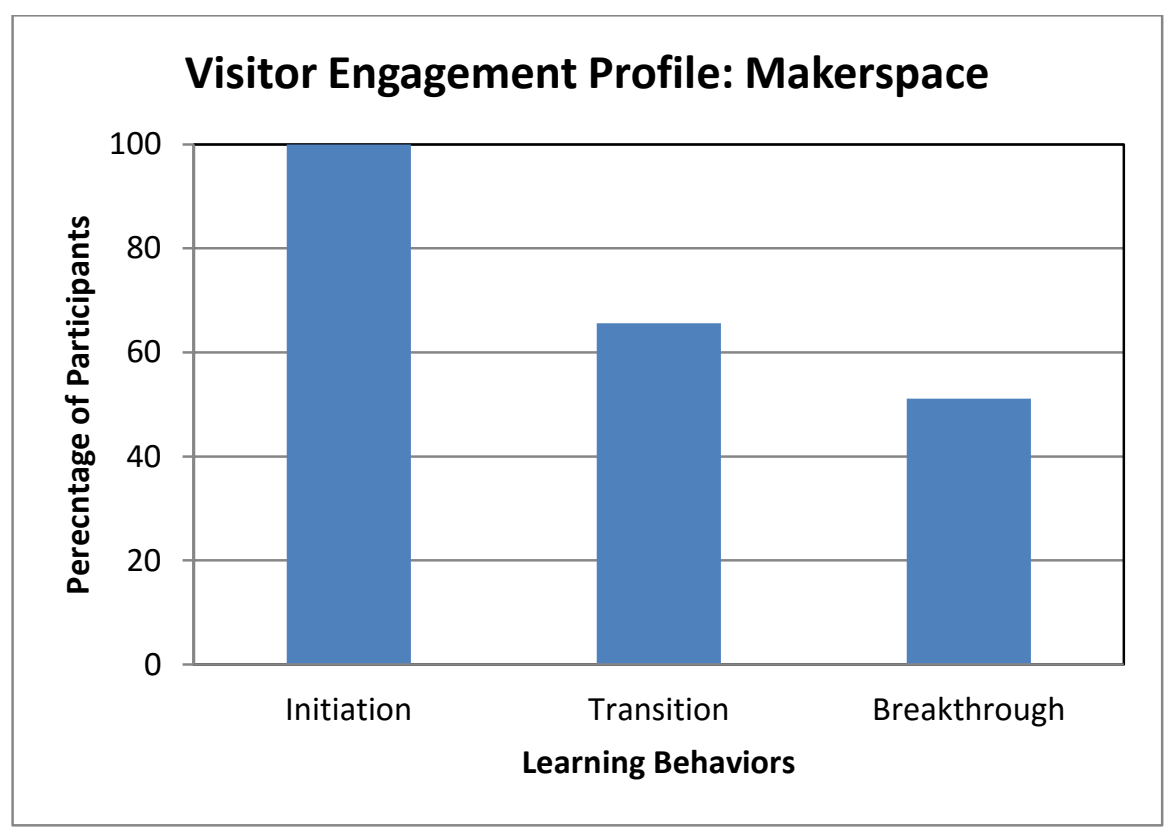

Figure 9. Overall VEP for the makerspace exhibit

Finally, one-sample chi-square analyses were conducted for the overall breakthrough behavior level for the makerspace as a whole as well as three of the 
individual activities. The hypothesized proportion of visitors that should reach breakthrough was $50 \%$, or 0.5 , based on the literature (Barriault, 2008). Table 11 shows the chi-square test statistics for the overall breakthrough category as well as the breakthrough level when separated based on the three activities with the most participants (hydraulics, squishy circuit, and stop-motion animation).

Table 11

Chi-Squared Test Statistics and Significance for Learning Behaviors $(d f=1)$

\begin{tabular}{|c|c|c|c|c|c|}
\hline & $N$ & $\begin{array}{c}\text { Does Not } \\
\text { Exhibit } \\
\text { (observed) }\end{array}$ & $\begin{array}{l}\text { Exhibits } \\
\text { (observed) }\end{array}$ & Chi-Square & Asymp. Sig. \\
\hline Breakthrough Behavior & 90 & 44 & 46 & 0.044 & 0.833 \\
\hline Breakthrough-Hydraulics & 15 & 11 & 4 & 3.267 & 0.071 \\
\hline $\begin{array}{l}\text { Breakthrough-Squishy } \\
\text { Circuit }\end{array}$ & 37 & 18 & 19 & 0.027 & 0.869 \\
\hline $\begin{array}{l}\text { Breakthrough-Stop } \\
\text { Motion Animation }\end{array}$ & 24 & 7 & 17 & $4.167 *$ & 0.041 \\
\hline
\end{tabular}

Although the overall makerspace exhibit did have more than 50\% breakthrough, with 46 out of 90 visitors exhibiting breakthrough behaviors, the results were not statistically significant, $\chi^{2}(1, N=90)=0.044, p=0.833$. However, when we look at specific activities, we can see that for the Stop Motion Animation activity, the results were statistically significant, $\chi^{2}(1, N=24)=4.167, p=0.041$. The effect size of 0.174 indicates that the observed frequencies for breakthrough behavior during the stop motion animation activity deviated slightly from the expected frequencies in a positive direction, meaning that visitors were statistically more likely to exhibit breakthrough behavior for this activity than the predicted level of 50\%. While the sample sizes of individual activities are small, this analysis indicates that the type of activity occurring within the makerspace can influence the amount of breakthrough behavior that visitors experience. 


\section{Summary}

Both quantitative and qualitative data were used to answer three research questions related to engagement levels in a children's museum makerspace. The first research question was focused on answering at what levels children engage with the makerspace exhibit in the museum. Data from the observation protocol were used to create the Visitor Engagement Profiles (VEPs) and descriptive analyses were run using means, standard deviations, frequencies, and percentages. The field notes were used to contextualize the quantitative data. The VEP for the overall makerspace $(N=90)$ showed that $51.1 \%$ of visitors reached the breakthrough level of behavior, and $65.8 \%$ reached the transition level.

Individual makerspace activities were also analyzed. The VEP for the Hydraulics activity $(N=15)$ showed just $26.6 \%$ of visitors reaching breakthrough level. Qualitative data demonstrated that breakthrough was difficult because parts of the activity were confusing and facilitators often did a poor job of encouraging breakthrough behaviors by the visitors, which led to boredom and eventually exiting the exhibit. The VEP for the Squishy Circuit Activity $(N=37)$ showed that $51.1 \%$ of visitors reached breakthrough levels of behavior. Qualitative data demonstrated that visitors experienced high levels of engagement due to the immersive nature of the stations for this activity. It also showed, however, that misconceptions were also an issue with this exhibit, and that the inquiry style of learning resulted in those misconceptions going unaddressed during the visitor experience. The VEP for the Stop Motion Animation Activity $(N=24)$ showed over $70 \%$ of visitors reached the breakthrough level. Qualitative data showed that teamwork, roleplaying, and planning in this activity were crucial, and these qualities helped to support 
higher levels of breakthrough by encouraging visitors to seek/share information with each other and refer to past experiences.

Overall, engagement was also influenced by the type of dialogue used by parents and facilitators. Positive dialogue, which included words of encouragement and questions prompting further thought and discussion, often led to higher levels of engagement. Negative dialogue, on the other hand, often led to disengagement and a loss of interest in the exhibit. Facilitators also influenced engagement based on whether they used scientific language, whether they had the content and pedagogical background to run the activity, and whether they themselves were engaged in the activities that they were running. Finally, the space itself influenced engagement. When there were extra distractions in the museum, visitors were less likely to remain in the exhibit and reach breakthrough.

The second research question was focused on answering what relationships exist between engagement level and age and gender. To answer this question, data from the observation protocol were used to run correlation and statistical significance tests with cross-tabs analysis. The field notes were used to contextualize the quantitative data. Young children (ages 0-6) made up 34.4\% of visitors, whereas children (ages 6-10) made up $62.2 \%$ of the visitors. The remaining visitors were classified as pre-teen (ages 11-13). Of the 90 participants, $51.1 \%$ were male. Overall, males were more likely to engage in breakthrough behaviors than females, though the results were not statistically significant. Males were statistically more likely at the 0.5 level to demonstrate the "referring to past experiences" behavior than females.

Qualitative data showed that both genders showed excitement but in different ways. Females were more likely to show excitement when sharing their creations with 
others, especially their parents. Meanwhile, males exhibited more hypothesis-testing behavior, and the use of prior knowledge to help determine what would happen next was often a factor in their excitement. Males and females also handled frustration and difficult tasks in different ways. Males were more likely to exit the exhibit when confronted with a difficult task or frustration, whereas females were more likely to be patient and persistent. However, when given the option to stay and explore or leave, females were more likely to leave the exhibit.

Aside from gender, the VEP according to age showed that young children were more likely to exhibit transition behaviors but children were more likely to exhibit breakthrough behaviors, though the results were not significant. Correlation coefficients showed a statistically significant relationship between transition and breakthrough level behaviors, meaning that those who reached the transition level were statistically more likely to reach the breakthrough level. Qualitative data suggested that young children were more likely to observe the activity rather than do the activity. Young children were also more likely to communicate with their parents or the facilitators compared to children visitors who were more likely to communicate with their peers. Finally, children visitors were more likely to use fixed-mindset or negative language while in the exhibit.

The third research question was focused on answering how the makerspace levels in the museum compare to engagement levels in traditional museum exhibits. Data from the observation protocol were used to create the overall Visitor Engagement Profile (VEP) and chi-square analysis was run. The VEP was then compared to what the literature has determined to be the expected engagement level based on level of interactivity and previous studies using the observation protocol instrument. The field 
notes were used to contextualize the quantitative data. Previous research shows that exhibits like a makerspace which are designed to involve visitors in hypothesis testing and meaning making should produce a VEP with at least $50 \%$ of visitors demonstrating breakthrough behaviors (Barriault, 2008). In this research, the VEP for the overall makerspace was $51.1 \%$ of participants exhibiting breakthrough behaviors. While this was not statistically significant, it did match the hypothesized portion of visitors to reach breakthrough. Among the individual activities, stop motion animation demonstrated statistical significance with breakthrough behaviors, meaning that visitors were statistically more likely to exhibit breakthrough behavior for this activity than the predicted level of 50\%. Qualitative data suggested that the observations from the makerspace aligned well with the context-specific framework developed for science centers. 


\section{CHAPTER V \\ SUMMARY, CONCLUSIONS, AND RECOMMENDATIONS \\ Overview of the Study}

Measuring the impact of informal learning environments can be very difficult because instruments tend to be developed for the formal learning environment and so are not always as effective in an informal setting. Yet museums often base exhibit decisions on feedback from visitors or parents, or on trends in other museums (Family Learning Forum, 2013). This is the case with makerspaces, which have been growing in popularity and are increasingly being added to museums, libraries, and schools (Broadbean, 2014). As the makerspace movement continues to grow, it is important to identify whether there are useful benefits for including makerspaces, which tend to be a resource- and timeintensive exhibit in Children's Museums (Halverson \& Sheridan, 2014). Given the relatively new inclusion of makerspaces in Children's Museums and the lack of research that currently exists, a number of questions arise. Understanding the level of engagement with the makerspace will help us to better understand how learning might be occurring in museum makerspaces and provide guidance for future exhibits to be effective without the need for programmatic and staff-intensive scaffolding. Additionally, understanding how demographic characteristics such as age and gender influence engagement outcomes will provide insight into the need for potential interventions or models for success. Therefore, the purpose of this concurrent mixed methods study was to better understand engagement 
and learning by converging both quantitative and qualitative data. In the study, an observation protocol developed by Barriault and Pearson (2010) known as the Visitor Based Learning Framework (VBLF) was used to measure the engagement levels of children in a museum makerspace. At the same time, field notes were collected to explore the context in which this engagement takes place. The following research questions were used to guide this study:

1. At what levels, based on the VBLF, do children engage with the makerspace exhibit in the museum?

2. What relationships exist between engagement levels and age and gender?

3. How do makerspace engagement levels compare to average engagement levels of traditional museum exhibits?

This study used a mixed methods design to examine children's engagement levels and science learning when they participate in a makerspace exhibit. In the case of this study, mixed-methods is necessary in order to provide context to the quantitative data collected from the observation protocol. The instrument used in this study was a Visitor Based Learning Framework developed first by Barriault (1999) and then revised by Barriault and Pearson (2010), which categorizes students' stages of learning (whether they are at the initiation, transformation, or breakthrough stages of learning) as they engage with a museum exhibit.

For this study, the focus was specifically centered on the makerspace exhibit. The observation engagement data was compiled in Excel and then transferred to the Statistical Program for the Social Sciences (SPSS) data system for analysis. In order to answer the research questions posed in the study, descriptive data analyses such as means, standard 
deviations, frequencies, and percentages were conducted. Additionally, statistics such as two-way contingency table analysis using crosstabs and chi-square analysis were run to determine correlations and statistical significance. These statistical tests were chosen because they follow nonparametric procedures. The crosstabs analysis was used to measure whether there was statistical significance between variables (i.e. initiation behavior, transition behavior, breakthrough behavior, gender, age group). The chi-square analysis was used to measure whether the proportion of individuals who fell into the engagement categories was equal to the hypothesized values, which are based on the average results from exhibits in which the observation protocol was used.

Field notes were collected, and direct dialogue was recorded when possible. The notes were reviewed and coded according to themes within each engagement level that emerged over the course of the observations. Initial coding themes focused on patterns of similarities and differences between male and female participants as well as young child and child participants. Additionally, because five different activities took place over the course of the data collection period, the researcher looked for themes within each of the activities. Field notes were also coded for specific examples and dialogue that aligned with the framework itself. Finally, themes emerged regarding facilitator/participant interactions as well as parent/child interactions and were coded thusly.

\section{Summary of Findings}

Both quantitative and qualitative data were used to answer three research questions related to engagement levels in a children's museum makerspace. The first research question was focused on answering at what levels children engage with the makerspace exhibit in the museum. The VEP for the overall makerspace $(N=90)$ showed 
that $51.1 \%$ of visitors reached the breakthrough level of behavior, and $65.8 \%$ reached the transition level. Individual makerspace activities were also analyzed. The VEP for the Hydraulics activity $(N=15)$ showed just $26.6 \%$ of visitors reaching breakthrough level. Qualitative data demonstrated that breakthrough was difficult because parts of the activity were confusing and facilitators often did a poor job of encouraging breakthrough behaviors by the visitors, which led to boredom and eventually exiting the exhibit. The VEP for the Squishy Circuit Activity $(N=37)$ showed that $51.1 \%$ of visitors reached breakthrough levels of behavior. Qualitative data demonstrated that visitors experienced high levels of engagement due to the immersive nature of the stations for this activity. It also showed, however, that misconceptions were also an issue with this exhibit, and that the inquiry style of learning resulted in those misconceptions going unaddressed during the visitor experience. The VEP for the Stop Motion Animation Activity $(N=24)$ showed over $70 \%$ of visitors reached the breakthrough level. Qualitative data showed that teamwork, role-playing, and planning in this activity were crucial, and these qualities helped to support higher levels of breakthrough be encouraging visitors to seek/share information with each other and refer to past experiences.

Overall, engagement was also influenced by the type of dialogue used by parents and facilitators. Positive dialogue, which included words of encouragement and questions prompting further thought and discussion, often led to higher levels of engagement. Negative dialogue, on the other hand, often led to disengagement and a loss of interest in the exhibit. Facilitators also influenced engagement based on whether they used scientific language, whether they had the content and pedagogical background to run the activity, and whether they themselves were engaged in the activities that they were running. 
Finally, the space itself influenced engagement. When there were extra distractions in the museum, visitors were less likely to remain in the exhibit and reach breakthrough.

The second research question was focused on answering what relationships exist between engagement level and age and gender. Young children (ages 0-6) made up $34.4 \%$ of visitors, whereas children (ages $6-10$ ) made up $62.2 \%$ of the visitors. The remaining visitors were classified as pre-teen (ages 11-13). Of the 90 participants, $51.1 \%$ were male. Overall, males were statistically more likely at the 0.05 level to demonstrate the "referring to past experiences" behavior than females. Qualitative data showed that both genders showed excitement but in different ways. Females exhibited "seeking and sharing" behavior by showing excitement when sharing their creations with others, especially their parents. Meanwhile, males exhibited hypothesis-testing behavior, and the use of prior knowledge to help determine what would happen next was often a factor in their excitement, based on the field notes.

Males and females also handled frustration and difficult tasks in different ways. The majority of visitors to exit the exhibit when confronted with a difficult task or frustration were males. Females were observed to have more patience and persistence when confronted with a difficult task or frustration. However, when given the option to stay and explore or leave, the field notes show that females were more likely to leave the exhibit than their male counterparts. Aside from gender, the VEP according to age showed that young child visitors exhibited transition behaviors more than child visitors, but child visitors exhibited breakthrough behaviors more than young child visitors, though the results were not significant. Correlation coefficients showed a statistically significant relationship between transition and breakthrough level behaviors, meaning 
that those who reached the transition level were statistically more likely to reach the breakthrough level. Qualitative data suggested that young child visitors exhibited the "observe the activity" initiation behavior whereas child visitors exhibited the "doing the activity" initiation behavior. Young child visitors also communicated with their parents or the facilitators more compared to child visitors, who were more likely to communicate with their peers based on field notes taken during the study. Finally, of the visitors who used fixed-mindset or negative language while in the exhibit, the majority were in the child age group.

The third research question was focused on answering how the makerspace levels in the museum compare to engagement levels in traditional museum exhibits. Previous research shows that exhibits like a makerspace which are designed to involve visitors in hypothesis testing and meaning making should produce a VEP with at least $50 \%$ of visitors demonstrating breakthrough behaviors (Barriault, 2008). In this research, the VEP for the overall makerspace was $51.1 \%$ of participants exhibiting breakthrough behaviors. While this was not statistically significant, it did match the hypothesized portion of visitors to reach breakthrough. Among the individual activities, stop motion animation demonstrated statistical significance with breakthrough behaviors, meaning that visitors were statistically more likely to exhibit breakthrough behavior for this activity than the predicted level of 50\%. Qualitative data suggested that the observations from the makerspace aligned well with the context-specific framework developed for science centers. 


\section{Conclusions}

One of the many challenges for museums is to find ways to encourage breakthrough behaviors in order to increase engagement. This is true whether it is an art museum or a science center or a children's museum. The observation protocol developed by Barriault (1998) has been used effectively for over a decade in a science center to measure engagement levels for exhibits. That information was then used to modify or update existing exhibits in order to create more breakthrough levels (Barriault \& Pearson, 2010). This study used the instrument in a children's museum makerspace in order to measure engagement levels in the makerspace exhibit, the influence of age and gender on engagement, and whether or not engagement levels were as expected for this type of exhibit based on previous research.

The study showed that while breakthrough engagement was where it was expected to be based on previous research, at more than $50 \%$ of the visitors entering the space reaching breakthrough engagement levels, the results for this study were not statistically significant. However, qualitative data helped provide insight into factors that influenced breakthrough levels, such as parent dialogue, interest in the activity, and facilitator knowledge. What this demonstrates is that similar to traditional museum exhibits, some activities in the makerspace will bring a majority of participants to the breakthrough level while others will not. Therefore, the findings also show that while this instrument can determine the effectiveness of individual makerspace activities in allowing participants to reach breakthrough engagement levels, it cannot determine the effectiveness of the makerspace overall without consideration for the individual activities taking place and other critical factors that are not assessed by the instrument. 
This study is consistent with the literature in terms of understanding the learner and understanding the museum setting. Research shows that learner motivation is a key component of the learner experience because it influences a visitor's engagement and interest (Paris, 1997). This study showed that visitors who were not motivated by or interested in the makerspace activities did not engage past the initiation level of engagement. Research also shows the importance of positioning the learner as a way to develop self-identity and persistence in learning (Bricker \& Bell, 2014). This study demonstrated that positive dialogue, whether from a facilitator or parent, was critical in encouraging persistence and breakthrough behavior within the makerspace. With regard to understanding the museum setting, research shows that the design of the exhibit can influence the visitor experience (Barriault \& Pearson, 2010). In this study, visitors who were not able to access the tables in the makerspace or who were distracted by other sections of the space that interfered with the learning environment did not reach breakthrough levels of engagement. Finally, there is no shortage of research on the critical role that the facilitator plays in the learning process of a museum exhibit, and this study provides even further evidence on how the facilitator can influence engagement simply by the ways in which she communicates with the visitor.

\section{Implications and Strategies}

Understanding how children are becoming engaged outside of the formal education environment is even more important given that high-stakes testing has led to a narrowing of curricular content, fragmentation of subject-area knowledge, and increased reliance on teacher-centered pedagogies ( $\mathrm{Au}, 2007)$. This can be accomplished by examining the level of engagement and learning that takes place within the makerspace to 
help us better understand how learning might be occurring in museum makerspaces and to provide guidance for future exhibits to be effective without the need for programmatic and staff-intensive scaffolding. Additionally, understanding how demographic characteristics influence engagement and learning outcomes will provide insight into the need for potential interventions or models for success. Museum staff who are already running makerspace programs, as well as those who are considering the addition of a makerspace to their museum, will find this study most beneficial. The paragraphs that follow will further explore the implication for museum educators and the implications for practice.

\section{Implications for Museum Educators}

As with any research study on museums, the reader ultimately wants to know the answer to one question: what should museums do with this research? For this study, the answer is relatively simple. The study shows that the observation protocol used in this study can determine the effectiveness of individual exhibit activities in terms of visitor engagement within a makerspace exhibit. Museum educators should use this information to help evaluate their own exhibits. When applied to a museum setting, this protocol can help educators identify strengths and weaknesses within exhibits. This information can then be used to highlight the positive engagement taking place and address the areas that need to be improved, whether that means re-evaluating a specific activity or changing the aesthetics or any other variety of exhibit improvement. Furthermore, the instrument protocol, when coupled with observations, can help museum educators determine the best forms of facilitation for the activities, which can help with training and professional development of museum staff members and the museum educators themselves. Not only 
is this process effective for any museum exhibit, but it is also useful in the context of makerspaces. Given that many museums are adding makerspaces without much knowledge of what will and will not be effective in the space, this instrument is crucial in order to run a successful exhibit and can provide great insight for planning purposes for museum educators.

\section{Implications for Practice}

The benefits of this observation protocol in practice are obvious. The instrument does not require extensive training in order for a researcher to be able to use it. It also does not require a lot of money to use the instrument in order to collect and analyze data. For small museums who cannot afford to hire external researchers or spend hours training their own employees, such an assessment tool is not only useful but a welcome change. Museums, evaluators, or other staff members can use this instrument without much training and collect a lot of information about how their visitors are engaging with their exhibits, which then allows them to adjust their programming accordingly. Because of this, the instrument ranks high in terms of practicality and effectiveness. Yet, issues still exist with using the instrument in practice.

The observation protocol used in this study was a great tool for determining engagement of the individual activities, but it failed to illuminate the importance that parent and facilitator interaction played in getting the participants to those higher engagement levels. It also failed to account for the importance of proper facilitator content and pedagogical knowledge in order to effectively run an exhibit activity. Children's museums who want to include makerspaces as part of their exhibit offerings should consider that these spaces do not yet engage young children and children at the 
breakthrough level in a way that is statistically significant. Museums should also consider that engagement is dependent upon how facilitators and parents interact with the child visitor to the space.

Furthermore, the gender barrier that is presented is a problem. Because females are less likely to reach breakthrough behavior levels than their male peers, and increased effort should be placed on removing this gender barrier. Facilitators should focus not only on getting girls into the space, which they seem willing to do on their own given the even split between male and female participants, but they should also encourage them to persist in what they are doing and recognize that they can use the tools and materials in the same way that their male peers can.

Finally, given that parent dialogue was also critical to the engagement level of the child, parents should be provided with clear guidelines from the facilitators in regards to how they should interact with their child while in the makerspace in order to achieve the highest learning behavior possible. This includes appropriate ways to question as well as using positive dialogue and knowing when it is and is not appropriate to help a child with a project. These findings demonstrate a need to further explore the ways in which parentchild and facilitator-child interactions affect engagement in museums. If these issues can be addressed, museums will be able to look to makerspaces as a prime example of how to achieve breakthrough learning behaviors among its visitors.

\section{Recommendations for Future Research}

Because makerspaces vary in their purpose and focus of activities, it would be difficult to find an instrument that would be effective in every makerspace. This study found that this observation protocol developed by Barriault (1999) was able to determine 
whether or not a makerspace activity was effective in allowing participants to reach that breakthrough level, but results depended on the individual activity. Therefore, it cannot at this time be used effectively to examine makerspaces overall without consideration for the individual activities taking place and other critical factors that are not assessed by the instrument at this time. For makerspaces that operate within museum, school, or other public settings, this instrument should be considered as a way to determine which activities will be effective in the makerspace setting. For more traditional makerspaces in which visitors determine their own activities based on their interests without facilitation from others, further study is needed to determine whether an instrument such as the observation protocol used in this study would be effective in that setting.

In conclusion, developing effective tools for informal learning environments is crucial to understanding the learning that takes place in these settings, since it is often influenced by factors other than what traditional formal assessment tools measure. The observation protocol used in this study was effective in measuring engagement but did not provide enough insight into why breakthrough behavior was not reached by more visitors. When individual activities were analyzed both quantitatively and qualitatively, it became clear how important the facilitator and parent were to the visitor reaching breakthrough behaviors. This study shows that makerspaces have the potential to provide high levels of breakthrough, but they cannot reliably do so without appropriate training for the facilitators in both content of the exhibit and appropriate pedagogical strategies. Since these spaces are often run by volunteers or part-time staff who may or may not have a background in education and pedagogical strategies, this presents a difficult dilemma for many museums. 
Future research should focus on how museums can implement appropriate training programs for their facilitators in order to improve their ability to run effective exhibits with high levels of breakthrough. The children's museum setting and makerspaces are also worthy of further study given that their exhibits and activities tend to be highly interactive, which should result in higher breakthrough levels of engagement. While this study fell short in terms of statistical significance for breakthrough, its significance for museum educators and for how the instrument is used in practice cannot be ignored. Museum educators can benefit greatly by using the findings in this study to shape their concepts and activities within their own makerspaces. Additionally, the study showed that this instrument was an effective tool in practice because it did not require extensive training or money, two components that small museums often lack, in order to collect and analyze the data.

Finally, future research should examine the gender barrier that exists in makerspaces in particular. Qualitative and quantitative data from this study both highlighted the skew of the makerspace setting toward male participants. Though there were almost an equal number of males and females participating, the males were more likely to reach breakthrough levels of engagement. Makerspace activities as well as the design of the space, which often consisted of workbenches, tools, and carpentry materials, could have created a hidden bias towards boys. This is something that facilitators and museums should be aware of, and it is a definite area for future research. Looking forward, the observation protocol has great potential for children's museums and makerspaces in terms of identifying exhibits which need intervention in order to operate at the highest level of breakthrough possible, but these interventions can only be effective 
if we have a better understanding of some of the components that influence breakthrough behavior in these spaces. 


\section{REFERENCES}

Anderson, D., Lucas, K., \& Ginns, I. (2003). Theoretical perspectives on learning in an informal setting. Journal of Research in Science Teaching, 40, 177-199.

Ash, D. (2003). Dialogueic inquiry in the life science conversations of family groups in a museum. Journal of Research in Science Teaching, 40, 138-162.

Ash, D. (2004). Reflective scientific sense-making dialogue in two languages: The science in the dialogue and the dialogue in the science. Science Education, 88, $855-884$.

Au, W. (2007). High-stakes testing and curricular control: A qualitative metasynthesis. Educational Researcher, 36(5), 258-267. DOI:10.3102/0013189X07306523

Azevedo, F.S. (2013). The tailored practice of hobbies and its implication for the design of interest-driven learning environments. Journal of the Learning Sciences, 22(3), 462-510.

Bailey, E.B. (2003). Researching museum educators' perceptions of their roles, identity, and practice. The Journal of Museum Education, 31(3), 175-197.

Bailey, L. M., Moris, L. G., Thompson, W. D., Feldman, S. B., \& Demetrikopoulos, M. K. (2016). Historical Contribution of Creativity to Development of Gifted Science Education in Formal and Informal Learning Environments. In Interplay of Creativity and Giftedness in Science (pp. 3-13). SensePublishers. 
Barriault, C. (1999). The science centre learning experience: A visitor-based framework. The Informal Learning Review, 35(1), 14-16.

Barriault, C. (2008). Supporting learning: Assessing the visitor learning experience through research in science centers. In Michelsen, C. (Ed.), Proceedings of the fourth Nordic network of researchers in science communication symposium, (pp. 15-24). Odense, Denmark: Print \& Sign.

Barriault, C. (2014). Visitor Engagement and Learning Behaviour in Science Centres, Zoos and Aquaria. Retrieved from http://espace.library.curtin.edu.au/R?func=dbin-jump-full\&object_id=226718.

Barriault, C. \& Pearson, D. (2010). Assessing exhibits for learning in science centers: A practical tool. Visitor Studies, 13(1), 90-106.

Bell, P., Lewenstein, B., Shouse, A.W., \& Feder, M.A. (Eds.). (2009). Learning science in informal environments: People, policies, and pursuits. A report of the National Research Council of the National Academies. Washington, D.C.: The National Academies Press.

Bodner, G.M. (1986). Constructivism: A theory of knowledge. Journal of Chemical Education, 63, 873-878.

Bodner, G., Klobuchar, M. \& Geelan, D. (2001). The many forms of constructivism. Journal of Chemical Education, 78(8), 1107.

Braund, M., \& Reiss, M.J. (Eds.). (2004). Learning science outside the classroom. London: Routledge-Falmer.

Bricker, L.A., \& Bell, P. (2014). "What comes to mind when you think of science? A perfumery!": Documenting science-related cultural learning pathways across 
contexts and timescales. Journal of Research in Science Teaching, 51(3), 260285.

Broadbean, J. (2014, July 6). Getting to know the makerspace movement in education. Retrieved February 20, 2016 from http://www.emergingedtech.com/2014/07/makerspace-movement-in-education/.

Bunce, D. (2001). Does Piaget still have anything to say to chemists? Journal of Chemical Education, 78(8), 1107.

Burchenal, M., \& Grohe, M. (2007). Thinking through art: Transforming museum curriculum. The Journal of Museum Education, 32(2), 111-122.

Callanan, M.A. (2012). Conducting cognitive developmental research in museums: Theoretical issues and practical considerations. Journal of Cognitive Development, 12(2), 137-151. DOI: 10.1080/15248372.2012.666730.

Castle, M.C. (2006). Blending pedagogy and content: A new curriculum for museum teachers. The Journal of Museum Education, 31(2), 123-132.

Claussen, S. \& Osborne, J. (2012). Bourdieu's notion of cultural capital and its implications for the science curriculum. Science Education, 97 (1), 58-79.

Conlon, T.J. (2004). A review of informal learning literature, theory, and implications for practice in developing global professional competence. Journal of European Industrial Training, 28(2/3/4), 283-295.

Creswell, J.W. (2003). Research design: Qualitative, quantitative, and mixed methods approaches. ( $2^{\text {nd }}$ ed.). Thousand Oaks, CA: Sage Publications. 
Crowley, K., \& Callanan, M. (1998). Describing and supporting collaborative scientific thinking in parent-child interactions. The Journal of Museum Education, 23(1), $12-17$.

DeBoer, G.E. (2000). Scientific literacy: Another look at its historical and contemporary meanings and its relationship to science education reform. Journal of Research in Science Teaching, 37(6), 582-601.

Department of Health, Education, and Welfare (1979). The Belmont report: Ethical principles and guidelines for the protection of human subjects of research. National Commission for the Protection of Human Subjects of BioMedical and Behavioral Research. Retrieved from http://www.hhs.gov/ohrp/policy/belmont.html.

Dewey, J. (1938). Experience and Education. New York, NY: Collier Books.

Dewitt, J.E. (2008). What is this exhibit showing you? Insights from stimulated recall interviews with primary school children. The Journal of Museum Education, 33(2), 165-173.

Dierking, L., \& Martin, L. (1997). Guest editorial. Science Education, 81, 629-631.

Downey, S., Delamatre, J., \& Jones, J. (2007). Measuring the impact of museum-school programs: Findings and implications for practice. The Journal of Museum Education, 32(2), 175-187.

Dreier, O. (2008). Psychotherapy in everyday life. Cambridge, UK: Cambridge University Press. 
Dudzinska-Przesmitzki, D., \& Grenier, R.S. (2008). Nonformal and informal adult learning in museums: A literature review. The Journal of Museum Education, $33(1), 9-22$.

Fadigan, K.A., \& Hammrich, P.L. (2004). A longitudinal study of the educational and career trajectories of female participants of an urban informal science education program. Journal of Research in Science Teaching, 41(8), 835-860.

Falk, J.H., \& Adelman, L.M. (2003). Investigating the impact of prior knowledge and interest on aquarium visitor learning. Journal of Research in Science Teaching, 40(2), 163-176.

Family Learning Forum (2013). Visitor evaluation project - Rethinking exhibitions. Retrieved February 20, 2016, from http://www.familylearningforum.org/rethinking-exhibitions/designingexhibits/modifying-exhibits.htm.

Felton, M.K., \& Kuhn, D. (2007). "How do I know?" The epistemological roots of critical thinking. The Journal of Museum Education, 32(2), 101-110.

Griffin, J. (2004). Research on students and museums: Looking more closely at the students in school groups. Science Education, 88(Suppl. 1), S59-S70.

Griffin, J., \& Symington, D. (1997). Moving from task-oriented to learning-oriented strategies on school excursions to museums. Science Education, 85, 454-481.

Gutwill, J. P. (2002). Gaining visitor consent for research: Testing the posted-sign method. Curator, 45(3), 232-238.

Gutwill, J.P. (2003). Gaining visitor consent for research: II. Improving the posted-sign method. Curator: The Museum Journal, 46, 228-235. 
Gutwill, J.P. (2008). Challenging a common assumption of hands-on exhibits: How counterintuitive phenomena can undermine inquiry. Journal of Museum Education, 33(2), 187-198.

Hall, R., \& Schaverien, L. (2001). Families' engagement with young children's science and technology learning at home. Science Education, 85, 454-481.

Halverson, E.R., \& Sheridan, K.M. (2014). The maker movement in education. Harvard Educational Review, 84(4), 495-504.

Harkins, H. (2011). Applying an observational framework in exhibit evaluation. Paper presented at the Visitor Studies Association Annual Conference, Chicago, IL.

Harlow, D.B. (2012). The excitement and wonder of teaching science: What pre-service teachers learn from facilitating family science night centers. Journal of Science Teacher Education, 23(2), 199-220.

Harré, R., Moghaddam, F.M., Cairnie, R.P., Rothbart, D., \& Sabat, S.R. (2009). Recent advances in positioning theory. Theory and Psychology, 19, 5-31.

Hatch, M. (2014). The maker movement manifesto. New York: McGraw-Hill.

Hidi, S., \& Renninger, K.A. (2006). The four-phase model of interest development. Educational Psychologist, 41(2), 111-127.

Hofstein, A., \& Rosenfeld, S. (1996). Bridging the gap between formal and informal science learning. Studies in Science Education, 28, 87-112.

Hohenstein, J., \& Tran, L.U. (2007). Use of questions in exhibit labels to generate explanatory conversation among science museum visitors. International Journal of Science Education, 29, 1557-1580. 
Holland, D. \& Leander, K. (2004). Ethnographic studies of positioning and subjectivity: An introduction. Ethos, 32(2), 127-139.

Honey, M., \& Kanter, D. (2013). Design-Make-Play: Growing the next generation of science innovators. New York: New York Hall of Science.

Jarman, R. (2005). Science learning through scouting: An understudied context for informal science education. International Journal of Science Education, 27, 427450.

Jeffery-Clay, K.R. (1998). Constructivism in museums: How museums create meaningful learning environments. The Journal of Museum Education, 23(1), 3-7.

Jensen, E. (2014). The problems with science communication evaluation. JCOM: Journal of Science Communication, 13(1). Retrieved from http://jcom.sissa.it/sites/default/files/documents/JCOM_1301_2014_C04.pdf.

Jones, L.S. (1997). Opening doors with informal science: Exposure and access to our underserved students. Science Education, 81, 663-677.

Kafai, Y.B., Fields, D.A., \& Searle, K.A. (2014). Electronic textiles as disruptive designs: Supporting and challenging maker activities in schools. Harvard Educational Review, 84(4), 532-556.

Kidder, L.H. (1981). Selltiz, Wrightsman \& Cook's Research Methods in Social Relations ( $4^{\text {th }}$ ed.) Austin, TX: Holt, Rinehart and Winston.

Lincoln, Y.S., \& Guba, E.G. (1985). Naturalistic Inquiry. Thousand Oaks, CA: Sage.

Lowery-Bretz, S. (2001). Novak's theory of education: Human constructivism and meaningful learning. Journal of Chemical Education, 78(8), 1107.

Lucas, K.B. (2000). One teacher's agenda for a class visit to an interactive science center. 
Science Education, 84, 524-544.

Lucas, A.M., McManus, P., \& Thomas, G. (1986). Investigating learning from informal sources: Listening to conversations and observing play in science museums. International Journal of Science Education, 8(4), 341-352.

Luke, F.T., Stein, J., Foutz, S., \& Adams, M. (2007). Research to practice: Testing a tool for assessing critical thinking in art museum programs. The Journal of Museum Education, 32(2), 123-135.

Mack, N., Woodsong, C., MacQueen, K.M., Guest, G., \& Namey, E. (2005). Qualitative research methods: A data collector's field guide. Retrieved from http://www.fhi360.org/sites/default/files/media/documents/Qualitative\%20Resear ch\%20Methods\%20-\%20A\%20Data\%20Collector's\%20Field\%20Guide.pdf.

Martin, L., \& Toon, R. (2003). Balancing acti: Activity theory applications to exhibit designs. The Journal of Museum Education, 28(2), 14-19.

Merriam, S.B. (1998). Qualitative research and case study applications in education. ( $2^{\text {nd }}$ ed.). San Francisco, CA: Jossey-Bass.

National Science Board (1998). Science and engineering indicators-1998. Arlington, VA: National Science Foundation (NSB-98-1).

National Science Board (2000). Science and engineering indicators-2000. Arlington, VA: National Science Foundation (NSB-00-1).

National Science Board (2002). Science and engineering indicators-2002. Arlington, VA: National Science Foundation (NSB-02-1).

Noel-Storr, J., Mitchell, S., \& Drobnes, E. (2010). NASA science served family style. Bulletin of the American Astronomical Society, 42, 504. 
Nurrenbern, S. (2001). Piaget's theory of intellectual development revisited. Journal of Chemical Education, 78(8), 1107.

Pajak, E. F., Stotko, E., \& Masci, F. (2007). Honoring diverse styles of beginning teachers. In Ornstein, A. C., Pajak, E. F., \& Ornstein, S. B. (Eds.) (5 $5^{\text {th }}$ ed.), Contemporary issues in curriculum, (pp. 110-117). Upper Saddle River, NJ:

Pearson.

Paris, S.G. (1997). Situated motivation and informal learning. The Journal of Museum Education, 22(2/3), 22-27.

Partnership for $21^{\text {st }}$ Century Skills (2011). Framework for $21^{\text {st }}$ century learning. Retrieved from http://www.p21.org/storage/documents/1__p21_framework_2pager.pdf.

Persson, P.-E. (2000). Community impact of science centers: Is there any? Curator: The Museum Journal, 43, 9-17.

Phipps, M. (2010). Research trends and findings from a decade (1997-2007) of research on informal science education and free-choice science learning. Visitor Studies, 13(1), 3-22.

Piaget, J. (1954). The construction of reality in the child. New York: Basic Books.

Ramey-Gassert, L., Walberg, H.J., III, \& Walberg, H.J. (1994). Reexamining connections: Museums as science learning environments. Science Education, 78, $345-363$.

Rennie, L.J., \& Johnson, D.J. (2007). Visitors' perceptions of changes in their thinking about science and technology following a visit to a science center. Visitor Studies, $10,168-177$. 
Rennie, L.J., \& McClafferty, T.P. (1995). Science centers and science learning. Studies in Science Education, 27, 53-98.

Ritchhart, R. (2007). Cultivating a culture of thinking in museums. The Journal of Museum Education, 32(2), 137-153.

Rix, C., \& McSorley, J. (1999). An investigation into the role that school-based interactive science centers may play in the education of primary-aged children. International Journal of Science Education, 21, 577-593.

Root-Bernstein, R., \& Root-Bernstein, M. (2005). Science museums and the arts of imaginative thinking. The Journal of Museum Education, 30(1), 3-8.

Schauble, L., Leinhardt, G., \& Martin, L. (1997). A framework for organizing a cumulative research agenda in informal learning contexts. The Journal of Museum Education, 22(2/3), 3-8.

Sedzielarz, M. (2003). Watching the chaperones: An ethnographic study of adult-child interactions in school field trips. The Journal of Museum Education, 28(2), 20-24.

Sheridan, K.M., Halverson, E.R., Brahms, L., Litts, B.K., Jacobs-Priebe, L., \& Owens, T. (2014). Learning in the making: A comparative case study of three makerspaces. Harvard Educational Review, 84(4), 505-531.

Silvertown, J. (2009). A new dawn for citizen science. Trends in Ecology and Evolution, 24(9), 467-471.

Stocklmayer, S. \& Gilbert, J.K. (2002). New experiences and old knowledge: Towards a model for the personal awareness of science and technology. International Journal of Science Education, 24, 835-858. 
Stone, A. (2008). Measuring progress toward mission. ASTC Dimensions, May/June Issues, 11.

Storksdieck, M., Jones, M.C., Falk, J.H., \& Alpert, C.L. (2002). Public understanding of science: A literature review. Anapolis, MD: Institute for Learning Innovation.

Tulley, A., \& Lucas, A.M. (1991). Interactive with a science museum exhibit: Vicarious and direct experience and subsequent understanding. International Journal of Science Education, 13(5), 533-542.

U.S. Office for Human Research Protections (2009). Code of Federal Regulations:

Protection of human subjects. Retrieved from

http://www.hhs.gov/ohrp/humansubjects/guidance/45cfr46.html.

Visscher, N., \& Morrissey, C. (2010). Use and effectiveness of discovery carts at the Pacific Science Center. Paper presented at the Visitor Studies Association Annual Conference, Phoenix, AZ.

Vogt, W.P., Gardner, D.C., \& Haeffele, L.M. (2012). When to use what research design. New York City, NY: The Guilford Press.

Worts, D. (2006). Measuring museum meaning: A critical assessment framework. The Journal of Museum Education, 31(1), 41-49.

Zimmerman, H.T., Reeve, S., \& Bell, P. (2008). Distributed expertise in a science center: Social and intellectual role-taking by families. The Journal of Museum Education, 33(2), 143-152.

Zoldosova, K., \& Prokop, P. (2006). Education in the field influences children's' ideas and interest toward science. Journal of Science Education and Technology, 15(3), 304-313. 
APPENDIX A

\section{VISITOR BASED LEARNING FRAMEWORK} OBSERVATION PROTOCOL 


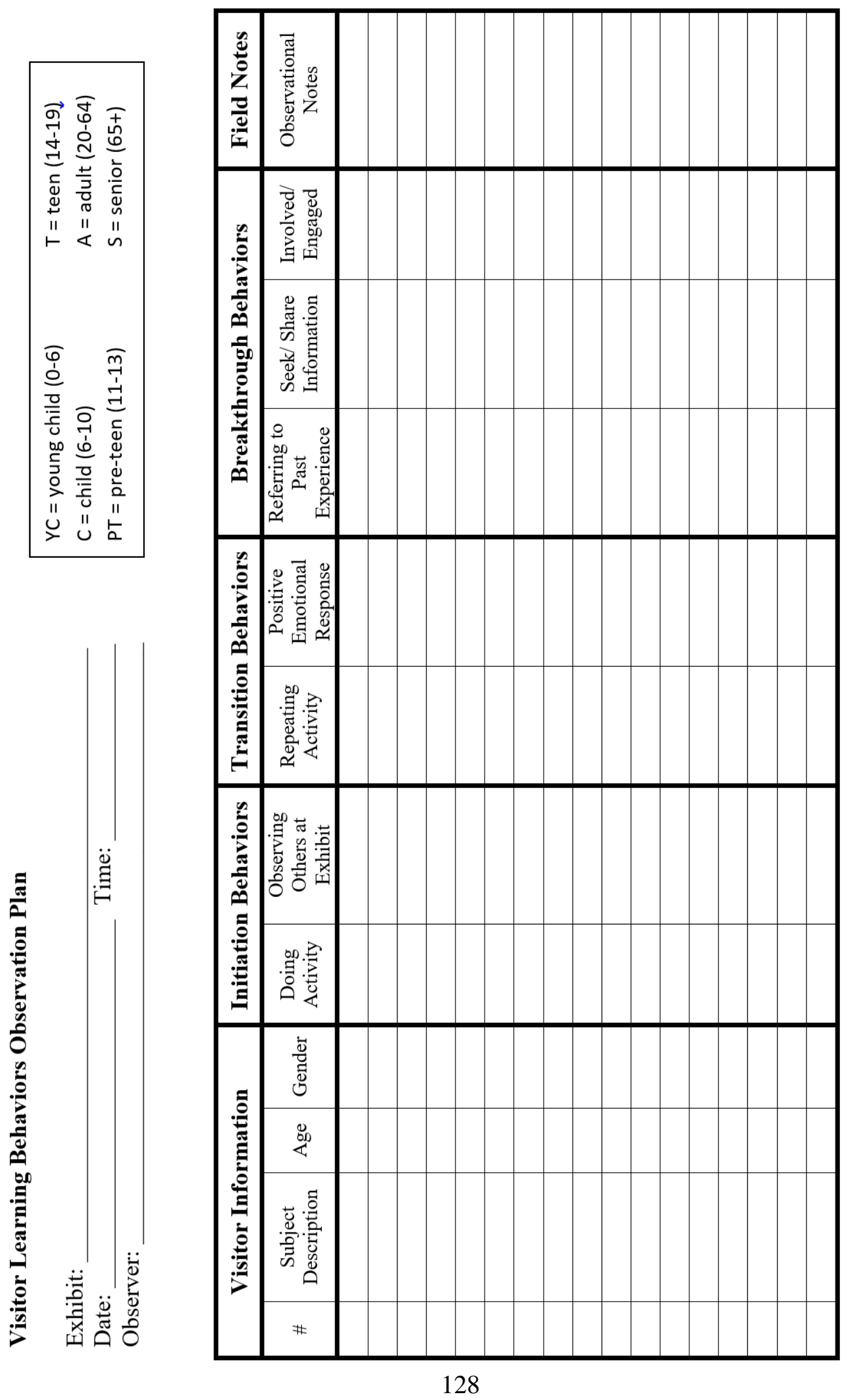




\section{APPENDIX B \\ MAKERSPACE CODED FIELD NOTES}

FOR VISITOR 13 


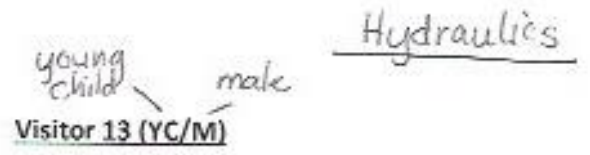

kid was here last weck for the pollinator experiment, returned this week to see It

what was new. How do $T$ do this? He says. The volunteer explains what is

happening. He takes the hydraulic system in his harids and moves both sides of the syringe. And you can keep exploring says the volunteer. I want to try it with the big one says the boy, he replaces the small syringe with a big one. Okay, says the T3

adult, now push this one. he does. wow says the boy. it just kecps working. That is B5

what we call hydraulics says the volunteer. Well that is an excavator and that is a

chair says the boy. Yos, says the volunteer. All of these machines move for us usine $T 4$ using hydraulics. he goes back to playing with the syringes, trying differcnt combinations of small and big oncs. Can you push the air out of this one, he says. Sure, says the volunteer. The aduit watches. Uh oh, there is still some water in here. What do you think will happen? says the adult. He is trying to pull the end out of one of the syringes that is connected to the one with water. I don't know ho says. Well lut's find out she says. He puts a full big one onto a small one. Boy 1 think I should get some tubing and stuff for home says the adult to the volunteer.

The kid continues to play. The kid puts the tube in the beaker and then pushes the syringe to cmpty the water. Good job says the adult. Very smart. she says. Now he is transferring water from one beaker to another by adding it to a syringe and then releasing it into another beaker. They have moved away from the topic of hydraulics, for now. That's all this con take because this one is full, he says. Okay, I sent mom a picture and told her you are learning about hydraulics, says the adult. So you uscd a little one, and it didn't really fill up the big one did it? soys the volunteer. How many times do you think it will take the little one to fill the big one? she says. I don't know he says. Do you have a gucss or prediction? she says? He 87 seems relicent to guess. It is okay if your gucss isn't right she says. We are going to test it out. I'm going to guess 7 she says. Okay, 1 guess 3 he says. Great, lel's try it. He proceeds to add three small syringes of water to the big one to see if it will fill the big one. He continues to fill the big syringe. You are already at 5 says the volunteer. Keep going says the adult. How many have we added now says the voluntecr? We had 5 and we added 1 more, how many is that? He looks confused.

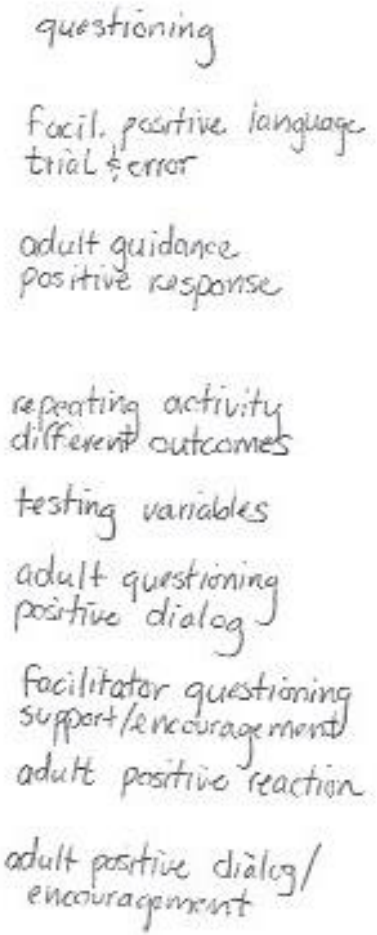

adut encovragement communication wo facilitator confusion 
Want to count my fingers she says? yes, he nods. He counts and then he says 6 . glill not full still not full says the volunteer. They keep adding, up to 10 . Wow, he says. So, one big one is the same as 10 little ones says the adult. Yeah, he says. That was a lot of water. I want to do all of them, he says. You guys can keep exploning however lang you want says the volunteer. If you are ready to move onto the next activity we can do that, but let him explore however long he wants says the volunteer to the adult. Obviously this has capturced his attention says the adult, referring to the hydraulic system example. He continues to play with the water and syringes. Hey, he says to the voluntcer, guess what! This thing just popped out! (referring to the back of the syringe). On nol Why do you think that happenced, says the volunteer? I'm not sure he says. Do you need morc tubes, asks the voluntecr? Yeah, he says. Then he pushed the tube and the back of the syringe popped off. They all jumped. Wow, did you know it would do that? says the volunteer? no he said. Did you know water could be that strong she says? Nol he exclamed. Because it was full and you were pushing it, there was pressurc building up and it had nowhere to go so it pushed the back orr the syringe, said the volunteer. It's like a watcr dart gun he said. Yeah, she says. he emplics it a little then connects a tubc and another syringe and is back to exploring the hydraulic system example. He grabs the extra tubing. I'll put this one on thesc, he says, and I'll try this one on these. He has three systems going at the sarne time. Okay, says the adult, let's try one more. Last time. $\mathrm{t}$ think we should empty them (the syringes) into there (the bcaker of water) and then go find something clse to do says the adult. Okay, he says. They move to the origarni station. A new volunteer takes a syringe and attaches it to the origami. What we do here is we use hydraulics to make the origami move he says. The syringe back paps off, and the boy says "that's what happened when I was over there (points to hydraulic system) station." So, says the volunteer, you can make some origami and then use hydraulics to make it move. Do you want to try? sure, says the boy. Had trouble with the origami, so he ended up leaving for another activity.

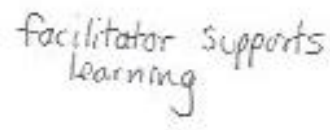

fiustration = exit the exlibit 


\section{APPENDIX C \\ LIST OF EMERGENT CODES}

WITH COMMENTS 


\section{Behavior Levels represented significantly:}

(in the coding and themes below, "V" stands for "visitor")

\begin{tabular}{|l|l|}
\hline Initiation Level 1 & V54, V66, V64, V77 \\
\hline Initiation Level 2 & V2, V5, V17, V26, V61, V81, V22, V33, \\
& V63, V41, V20 \\
\hline Transition Level 3 & V2, V14, V26, V53, V72 \\
\hline Transition Level 4 & $\begin{array}{l}\text { V4, V73, V80, V86, V3, V13, V36, V63, } \\
\text { V15, V90 }\end{array}$ \\
\hline Breakthrough Level 5 & V48, V86, V13, V32, V29, V75 \\
\hline Breakthrough Level 6 & V14, V61, V80, V13, V45, V29, V88, \\
& V11 \\
\hline Breakthrough Level 7 & V3, V13, V40 \\
\hline
\end{tabular}

Activity-specific patterns:

\begin{tabular}{|l|l|}
\hline \hline Activity-based themes for Hydraulics & $\begin{array}{l}\text { Easy shift from initiation to transition } \\
\text { because of the syringe activity, but } \\
\text { disengagement quickly after for the } \\
\text { origami activity (V14, V22), no } \\
\text { connection to overall concept-instead } \\
\text { just trying to move people through the } \\
\text { activities (V17, V22, V16) }\end{array}$ \\
\hline $\begin{array}{l}\text { Activity-based themes for Squishy } \\
\text { Circuits } \\
\text { types of dough (V26). Misconceptions are } \\
\text { easy to emerge and often went } \\
\text { unaddressed (V61). Visitors sometimes get } \\
\text { bored if working in groups because they } \\
\text { cannot directly experiment (V32). Lots of } \\
\text { opportunities for scientific method during } \\
\text { insulator/conductor activity (V38) } \\
\text { Obvious influence of different pedagogy } \\
\text { models on engagement (V30, V31, V46) }\end{array}$ \\
\hline $\begin{array}{l}\text { Activity-based themes for Stop-Motion } \\
\text { Animation }\end{array}$ & $\begin{array}{l}\text { Activity required lots of teamwork and } \\
\text { planning and often saw visitors pair up } \\
\text { with other visitors to work together (V70, } \\
\text { V80, V85). Also because of the focus on } \\
\text { making a video, it often led to a lot of role- } \\
\text { playing and acting-style conversations, } \\
\text { especially from the young child category } \\
\text { (V73, V80, V87, V88, V72) }\end{array}$ \\
\hline
\end{tabular}

\section{Parent/Child patterns:} -positive dialogue results in lots of questioning/critical thinking and engagement and an appreciation for effort (V2, V9, V3, V45, V63, V15, V52, V71, V90) 
-negative dialogue results in loss of interest and/or disengagement or moving onto the next exhibit (V5, V86, V36, V85, V28, V84, V1, V50), especially when time is an issue.

-parent encourages misconceptions and misrepresentations of science (V22)

\section{Facilitator patterns:}

-facilitator questioning leads to engagement of the visitor and recall of prior knowledge (V14, V48, V61, V81, V86, V32, V36, V10, V18) except when it is done in a way to discourage visitors from trying something, especially due to time constraints (V66)

-lack of facilitator guidance or knowledge can result in disengagement/misconceptions or even prevent breakthrough moments, and waiting for staff attention/assistance often leads to boredom and eventually the visitor leaving the exhibit (V14-hyd, V70-stopmotion, V73-stopmotion, V80stopmotion, V16-hyd, V53-squishy, V30-squishy) -facilitator/staff lack of interest (V17-hyd) -facilitator use of scientific language and praise/encouragement aids in reaching breakthrough by helping to encourage experimentation (V13, V67, V82-reengagement leads to breakthrough, V27)

-poor planning of space by having too many activities at once or resources out that are not available to use (V78) or resources that are not accessible to all visitors (V59)

\section{Young Child/Female themes:}

-lots of observing others (specifically a parent) doing the activity (V2)

-parent help is different than parent doing because it maintains engagement (i.e. sometimes the parent just starts doing all the work and the kid watches vs. other times when the kid asks the parent for help and the parent continues to question how the kid wants it instead of just doing it how they would do it) (V5, V73) -excitement at the process and/or finished product, with the desire to share with others, especially parents (V5, V81, V86)

-trial and error lead to success and persistence (V14, V86)

\section{Young Child/Male themes:}

-desire to share creation with parents or siblings (V32, V33, V45)

-bored or disengaged when not participating directly (parents/staff/other visitors are doing the activity) (V32, V51, V69)

-excitement at the process, positive reinforcement when hypothesis testing (V55) -parent help allows the visitor to be creative while the adult assembles the pieces of the creation (V63) 


\section{Child/Female themes:}

-communication with peers during activity and to share creation (V8, V39, V78) -problem solving, decision-making and persistence were important, as well as patience (V10, V40, V67, V68, V71)

-when given the option to disengage, she does (V15, V52)

-parent help is limited to assistance in creating materials rather than actually having parent do the work for the visitor (V39, V78, V88) or the child does not want parent help at all (V67)

-child uses negative/fixed mindset language (V40, V41, V82)

-nonverbal communication with staff and facilitators (V41)

-use of pre-made characters (V67, V71, V87)

-create own characters (V68, V75, V82, V83, V88)

\section{Child/Male themes:}

-communicating with peers, explaining or sharing information (V1, V11, V43)

-positive response after successful hypothesis testing (V11, V25, V27, V57)

-Distraction from task leads to disengagement, lack of involvement or interest in the purpose of the activity (V12, V44, V49)

-trouble/frustration with task leads to visitor leaving the exhibit (V18, V31, V44, V46, V62)

-child uses negative/fixed mindset language (V19) 
APPENDIX D

\section{DESCRIPTION OF MAKERSPACE ACTIVITIES}

BY THEME 


\section{Pollinators}

This makerspace theme consisted of two activities. In the first activity, a facilitator talked with the visitor about what pollinators are and how they can take nectar and move it to other flowers. They demonstrated this by having visitors try to use a straw to lift a sugary liquid from one container to another. Then visitors moved to the second activity, which allowed them to create their own version of a pollinator with the makerspace materials. In this case, there was no specific function to their creation except to resemble a pollinator. For example, many visitors chose to make bees or butterflies.

\section{Hydraulics}

This makerspace theme consisted of three activities. In the first activity, a set of books was placed on top of a small sized trash bag. The visitors used straws to blow air into the bag and try to lift the books. The facilitator talked to the visitor about this being an example of hydraulics. They talked about other real life examples such as elevators. Then visitors moved to the second activity. In this activity, visitors used two syringes that were connected to each other with a tube. One syringe was filled with water and the other was left empty. The visitors pushed on the water-filled tube to try and move the other end of the syringe. In later days, they used the movement of the empty syringe to push a cup across a line. Finally, in the last activity visitors could create an origami animal and then try to use balloons and syringes to use hydraulic concepts to move a part of the animal. For example, visitors might use a syringe to inflate a balloon and move the ears of a dog up and down.

\section{Open Lab}

This makerspace theme was more traditional to makerspaces. Visitors entered the space and had a series of workbenches with tools such as saws, hammers, etc. They had access to all of the materials in the space, including wood, electronics, cardboard, etc. There was no specific topic_-visitors could come in and build whatever they wanted as long as time and materials allowed.

\section{Squishy Circuits}

This makerspace theme consisted of three activities. In the first activity, a variety of conductive and insulating materials were laid out on the table. Visitors started at this activity and used a battery and wires to connect each material to see if the lightbulb would light up. Sometimes the facilitator told them the difference between conductors and insulators and then had them test the materials. Other times the facilitator waited for the visitor to discover the difference and then told them the terms. Visitors then sorted the materials into a pile of conductors and a pile of insulators. At the second activity, two 
types of dough were on the table. One dough was conductive and the other was an insulator. Again, visitors used a battery and wires to try to use the dough and light up the lightbulb. Finally, at the last activity, visitors could use both the conductive and insulating dough to build their own creation. They were also given LEDs with the goal of trying to get some part of their creation to light up. For example, visitors might build a creature with eyes that light up.

\section{Stop-Motion Animation}

In this makerspace activity, visitors entered the space to find printed 11x 17 background scenes from Harry Potter standing up on the tables. In front of the backdrops were preassembled Harry Potter characters attached to small stands which allowed them to stand up in front of the backdrops. At another table were blank characters that visitors could color and then assemble with brads to create their own characters. The table also had access to other makerspace materials such as crayons, hot glue guns, feathers, etc. Visitors entered the space and chose whether they wanted to use pre-assembled characters or create their own. Once they had their characters, they choose a backdrop for their story. A facilitator then explained that they would create a stop motion animation video by taking a series of pictures and having the characters make small movements so that when the pictures were put together, it would look like the characters were moving. Facilitators used old donated cell phones to take the photos and emphasized the importance of keeping the camera at the same height and location the whole time. Once the visitor finished with the pictures, the images were imported into a program called Monkey Jam. Visitors could manipulate the frames per second to speed up or slow down their movie. After giving the movie a title, it was saved and then later uploaded to the museum's YouTube channel for visitors to access at a later date. 